\title{
INTERACTIONS BETWEEN CATIONIC STARCH AND ANIONIC SURFACTANTS
}

\author{
Juha Merta
}

Dissertation for the degree of Doctor of Philosophy to be presented with due permission of the Department of Forest Products Technology for public examination and debate in Auditorium Ke 2 at Helsinki University of Technology (Espoo, Finland) on the 2th of March, 2001, at 12 noon.

Helsinki University of Technology

Department of Forest Products Technology

Laboratory of Forest Products Chemistry

Teknillinen korkeakoulu

Puunjalostustekniikan osasto

Puunjalostuksen kemian laboratorio 
Distribution:

Helsinki University of Technology

Laboratory of Forest Products Chemistry

P.O.Box 6300

FIN-02015 HUT, Finland

Tel. +35894511

Fax +35894514259

ISBN 951-22-5328-3

ISSN 1457-1382

Picaset Oy

Helsinki 2001 


\title{
HELSINKI UNIVERSITY OF TECHNOLOGY
}

\author{
Author: Juha Merta \\ Name of the dissertation: Interactions between Cationic Starch and Anionic \\ Surfactants \\ Language of the dissertation: English \\ Translation in Finnish: Kationisen tärkkelyksen ja anionisten pinta-aktiivisten aineiden \\ väliset vuorovaikutukset
}

Date of the manuscript: 5.12 .2000

Compound dissertation

Department: Department of Forest Products Technology

Laboratory: Laboratory of Forest Products Chemistry

Field of research: Surface and Colloid Chemistry

Supervisor: Professor Per Stenius HUT professorship code: Puu-19

Pre-examiners: Professors Heikki Tenhu and Johan Sjöblom

Opponent: Professor Mats Almgren

\section{Abstract}

The interactions between cationic starch (CS) and anionic surfactants and their mixtures were studied by using surface tension, solution viscosity and dynamic light scattering (DLS) measurements. The composition and structure of the complexes of CS and surfactants were studied by using phase equilibrium determination, small-angle neutron scattering (SANS) and small-angle $\mathrm{X}$-ray scattering (SAXS) measurements.

Critical association concentrations $(c a c)$ are well below the critical micellisation concentrations $(\mathrm{cmc})$ of the surfactants. Associative phase separation occurs in extremely dilute systems when the charge ratio between the surfactants and the polymer is close to one.

The effect of mixing on micellisation of the binary surfactant solution can be described by taking into account the effects of the volume difference between the hydrocarbon chains.

The separated phase is a hydrophobic, viscous, gel-like complex containing less than $60 \mathrm{w} \%$ of water. With mixed surfactants, the more surface-active component is enriched in the complex phase. The gel is dissolved entirely or partly when excess surfactant is added with charge reversal mechanism.

The mixed micelles of alkanoates are prolate ellipsoids with the longer-chain surfactant enriched in the end-cups of the ellipsoid. In dilute solutions CS molecules have a helical conformation. The core of CS/surfactant aggregates is formed by surfactant monomers and the surrounded shell by CSchains. The structure of the complexes in dilute solutions resembles the inclusion complexes of amylose and surfactants.

The charge neutralized CS-surfactant complexes consists of lyotropic liquid crystalline phase. The mesophases are the same as the pure surfactants form without any added polymer (hexagonal, lamellar or cubic), but they form with CS at lower surfactant concentrations.

The gel-like CS-surfactant complex phases have high viscosity and elasticity. Their rheological behaviour is described by the Hershel-Bulkley model.

Keywords: Cationic starch, anionic surfactants, interactions, complexes, structure, surface tension, phase equilibrium, viscosity, SAXS, SANS, DLS UDC: $661.185 .22: 541.18: 535.36$

Distributed by / Publisher: Helsinki University of Technology

Laboratory of Forest Products Chemistry

Reports; Series A 12

ISBN/ISSN (HUT series): ISBN-951-22-5328-3, ISSN-1457-1382

$107+108$ Pages

Contacts of the author: E-mail Juha.Merta@hut.fi

The dissertation can be read at http://www.hut.fi/Yksikot/Kirjasto/Diss/ 


\section{Preface}

The experimental work of this thesis was carried out at the Laboratory of Forest Products Chemistry of Helsinki University of Technology 1992 - 2000.

The small-angle X-ray scattering measurements were performed at Laboratory of X-ray Physics, University of Helsinki by Dr. Mika Torkkeli.

Most of the small-angle neutron scattering measurements were carried out by Dr. Vasyl Garamus at SANS1 of GKSS Research Centre, Geesthacht, Germany. Also, some measurements were made at the Frank Laboratory of Neutron Physics in Joint Institute for Nuclear Research, Dubna, Russia by Dr. Alexander Kuklin.

I express my deepest gratitude to Professor Per Stenius for guiding me in my work on surface and colloid chemistry. I would like to thank him also for all the encouragement and the stimulating discussions over these years.

I would like to thank the personnel at the Laboratory of Forest Products Chemistry for the positive and dynamic working atmosphere. My special thanks are due to Tekla Tammelin, Hanna Iitti, Emma Matikainen and Ritva Kivelä for excellent technical assistance, and also Lic. Sc. (Tech.) Esa Pirttinen for inspiring discussions.

Financial support from Finnish Technology Development Centre (TEKES) and different industries, especially Raisio Chemicals Oy, Finland, is gratefully acknowledged.

Finally, I warmly thank my wife, Sinikka, and my daughters, Emmi and Noora, for their patience and understanding. 


\section{LIST OF PAPERS}

This thesis consists of a summary and the following papers, which will be referred to in the text by their Roman numerals

I. Merta, J.; Stenius, P. (1995), Interactions between cationic starch and anionic surfactants. 1. Phase equilibria and surface tensions. Colloid Polym. Sci. 273, 974 - 983.

II. Merta, J.; Stenius, P. (1997), Interactions between cationic starch and anionic surfactants 2.Viscosity and aggregate size in dilute solutions. Colloids Surfaces A: Physicochem. Eng. Aspects 122, $243-255$.

III. Merta, J., Pirttinen, E.; Stenius, P. (1999), Interactions between cationic starch and anionic surfactants. III. Rheology and structure of the complex phase. J. Dispersion Sci. and Techn. 20, 677 - 697.

IV. Merta, J. and Stenius, P. (1999), Interactions between cationic starch and mixed anionic surfactants. Colloids Surfaces A: Physicochem. Eng. Aspects 149, 367 - 377.

V. Merta, J., Torkkeli, M., Ikonen, T., Serimaa, R. and Stenius, P. (2001). The structure of cationic starch (CS)/anionic surfactant complexes studied by small angle X-ray scattering. Macromolecules. Accepted.

VI. Merta, J., Garamus, V., Willumeit, R., Kuklin, A. and Stenius, P. (2000). Determination of the structure of Complexes Formed by a Cationic Polymer and Mixed Anionic Surfactants by SANS. Langmuir 16, $10061-10068$. 
The following acronyms and abbreviations are used throughout this thesis:

\begin{tabular}{|c|c|}
\hline AOT & Sodium diethylhexylsulfosuccinate \\
\hline $\mathrm{C}_{10} \mathrm{TAB}$ & Decyltrimethylammonium bromide \\
\hline $\mathrm{C}_{12} \mathrm{TAB}$ & Dodecyltrimethylammonium bromide \\
\hline $\mathrm{C}_{14} \mathrm{TAB}$ & Tetradecyltrimethylammonium bromide \\
\hline $\mathrm{C}_{16} \mathrm{TAB}$ & Hexadecyltrimethylammonium bromide \\
\hline $\mathrm{C}_{12} \mathrm{TAC}$ & Dodecyltrimethylammonium cloride \\
\hline $\mathrm{C}_{\mathrm{n}} \mathrm{TAB}$ & Alkyltrimethylammonium bromide \\
\hline $\mathrm{cac}$ & Critical association concentration \\
\hline $\mathrm{CS}$ & Cationic starch \\
\hline cec & Critical electrolyte concentration \\
\hline $\mathrm{cmc}$ & Critical micellisation concentration \\
\hline $\mathrm{C}_{\mathrm{n}}(\mathrm{EO})_{\mathrm{m}}$ & Alkyl poly(oxoethylene ether) \\
\hline DDBS & Sodium dodecylbenzene sulphonate \\
\hline DLS & Dynamic light scattering \\
\hline DMSO & Dimethyl sulfoxide \\
\hline DP & Degree of polymerisation \\
\hline DS & Degree of substitution \\
\hline EHEC & Ethyl(hydroxyethyl) cellulose \\
\hline fwhm & Full width at half maximum height \\
\hline $\mathrm{HB}$ & Herschel-Bulkley model \\
\hline HY & Hyaluronate \\
\hline KDod & Potassium dodecanoate \\
\hline KOct & Potassium octanoate \\
\hline LPEI & Linear poly(ethyleneimine) chloride \\
\hline $\mathrm{NaCMA}$ & Sodium carboxymethylamylose \\
\hline $\mathrm{NaDe}$ & Sodium decanoate \\
\hline NaDod & Sodium dodecanoate \\
\hline $\mathrm{NaDXS}$ & Sodium dextrane sulphate \\
\hline
\end{tabular}


$\mathrm{NaEr}$

$\mathrm{NaHy}$

NaOct

$\mathrm{NaOl}$

$\mathrm{NaPa}$

$\mathrm{NaPal}$

NaPSS

NaPVS

NMR

PAA

PAMPS

PDADMAC

PEO

PMAA /NIPAM

PMAA

Polymer JR

PVAc

PS1

PS4

PSS

PVP

PVS

Reten

SANS

SAXS

SDS

SEM

SHS

SOS

STS

TEM
Sodium cis-13-docosenoate (sodium erucate)

Sodium hyaluronate

Sodium octanoate

Sodium cis-9-octadecenoate (sodium oleate)

Sodium poly(acrylate)

Sodium hexadecanoate (sodium palmitate)

Sodium poly(styrene sulphonate

Sodium poly(vinyl sulphate)

Nuclear magnetic resonance

Poly(acrylic acid)

Poly[2-(acrylamido)-2-methylpropanesulphonic acid]

Poly(diallylmethylammonium chloride)

Poly(ethylene oxide)

Poly(sodium methacrylate-co- $\mathrm{N}$-isopropylacrylamide)

Poly(sodium methacrylate)

Cellulose, 2-hydroxyethyl 2-[2-hydroxy-3-(trimethylammonio)propoxy]

ethyl 2-hydroxy-3-(trimethylammonio)propyl ether, chloride (9CI)

Poly(vinyl alcohol)

Poly(maleic acid-co-methyl vinyl ether)

Poly(maleic acid-co-butyl vinyl ether)

Poly(styrene sulphonate)

Poly(vinyl pyrrolidone)

Poly(vinyl sulphate)

Acrylamide/ -methacryloxyethyltrimethylammonium chloride copolymer

Small Angle Neutron Scattering

Small Angle X-ray Scattering

Sodium dodecyl sulphate

Scanning electron microscopy

Sodium hexadecyl sulphate

Sodium octyl sulphate

Sodium tetradecyl sulphate

Transmission electron microscopy 


\section{CONTENTS}

1. INTRODUCTION 1

2. THEORETICAL BACKGROUND 2

2.1. Characteristic of polyelectrolytes 2

2.2. Surface active agents $\quad 3$

2.2.1. General $\quad 4$

2.2.2. Micelle formation in aqueous solutions 4

2.2.3. Factors affecting the cmc and aggregation number 5

2.2.4. Mixed micellisation $\quad 6$

2.2.4.1. Modelling mixed surfactants systems 6

$\begin{array}{ll}\text { 2.2.4.2. Synergism in mixing of different surfactants } & 7\end{array}$

$\begin{array}{ll}\text { 2.3. Polymer-surfactant interactions } & 8\end{array}$

$\begin{array}{ll}\text { 2.3.1. Driving forces } & 8\end{array}$

2.3.2. Binding isoterms $\quad 9$

2.3.3. Strength of interaction $\quad 10$

2.3.4. Interactions between polyelectrolytes and oppositely charged surfactants 11

2.3.4.1. Influence of the surfactant 12

$\begin{array}{ll}\text { 2.3.4.2. Influence of the polyelectrolyte } & 13\end{array}$

$\begin{array}{ll}\text { 2.3.4.3. Effect of salt } & 14\end{array}$

2.4. Polyeletrolyte/surfactant complexes $\quad 14$

2.4.1. Phase behaviour of hydrophilic polymers with pure surfactant micelles 14

$\begin{array}{ll}\text { 2.4.1.1. Oppositely charged mixtures } & 15\end{array}$

2.4.2. Structure of complexes $\quad 16$

2.4.2.1. Conformation of surfactant molecules $\quad 16$

2.4.2.2. Conformation of the polyelectrolyte 18

2.4.3. Native starch-surfactant complexes 19

2.4.4. Polyelectrolyte-surfactant complexes with high range order 19

2.4.4.1. In organic solvents and in the solid state 19

$\begin{array}{ll}\text { 2.4.4.2. In aqueous solutions } & 20\end{array}$

2.5. Basics of the research methods used in this study 23

2.5.1. Surface tension $\quad 23$

2.5.2. Solution viscosity and rheology $\quad 25$

2.5.3. Dynamic light scattering (DLS) 30

2.5.4. Small angle X-ray scattering (SAXS) and small angle neutron scattering (SANS) 34

2.5.4.1. Basics of the theory of small angle scattering 34

2.5.4.2. Scattering by simple objects $\quad 36$

2.5.4.3. Structure analyses of ionic micelles with SANS 38

2.5.4.4. Determination of molecular structure of liquid crystals by SAXS 41 
3.1. Materials $\quad 42$

3.1.1. Cationic starch $\quad 42$

3.1.2. Surfactants 44

3.2. Methods $\quad 45$

3.2.1. Surface tension measurements $\quad 45$

$\begin{array}{ll}3.2 .2 \text {. Viscosity measurements } & 46\end{array}$

$\begin{array}{ll}\text { 3.2.3. Dynamic light scattering } & 47\end{array}$

3.2.4. Electrophoretic mobility 47

3.2.5. Phase equilibria determination $\quad 48$

$\begin{array}{ll}\text { 3.2.6. Rheology } & 48\end{array}$

3.2.7. Small angle X-ray scattering (SAXS) 48

3.2.8. Small angle neutron scattering (SANS) $\quad 49$

$\begin{array}{ll}\text { 3.3. Results and discussions } & 50\end{array}$

3.3.1. Critical association concentrations (cac) $\quad 50$

3.3.1.1. Effect of the chain length of the surfactant $\quad 50$

$\begin{array}{ll}\text { 3.3.1.2. Effect of the charge density of the polymer } & 51\end{array}$

3.3.1.3. Effect of the polyelectrolyte concentration $\quad 53$

3.3.1.4. Effect of the electrolyte concentrations $\quad 53$

3.3.2. Phase separation and redissolution $\quad 54$

3.3.2.1. Dissolution of CS-surfactant precipitates $\quad 56$

$\begin{array}{ll}\text { 3.3.3. Phase diagrams } & 57\end{array}$

3.3.4. Interactions between CS and mixed anionic surfactants $\quad 60$

$\begin{array}{ll}\text { 3.3.4.1. Micellisation in surfactant mixtures } & 60\end{array}$

$\begin{array}{ll}\text { 3.3.4.2. Critical association concentrations } & 63\end{array}$

$\begin{array}{ll}\text { 3.3.4.3. Phase separation and redissolution } & 65\end{array}$

3.3.4.4. Surfactant ratio in CS/mixed micelle complex phase $\quad 66$

$\begin{array}{ll}\text { 3.3.5. Structure of CS-surfactant systems in dilute solutions } & 67\end{array}$

$\begin{array}{ll}\text { 3.3.5.1. Mixed micelles } & 68\end{array}$

3.3.5.2. CS/surfactant complexes $\quad 72$

3.3.6. Charge neutralized CS-surfactant complexes $\quad 77$

3.3.6.1. Structure of pure surfactant mesophases and CS/surfactant complexes 77

3.3.6.2. Lattice constant and interparticle distances $\quad 82$

3.3.6.3. Geometry of polyelectrolyte oppositely charged surfactant aggregate phases $\quad 83$

3.3.7. Rheology of the complexes $\quad 86$

$\begin{array}{ll}\text { 3.3.7.1. General } & 86\end{array}$

3.3.7.2. Fitting of the results to simple rheological models $\quad 88$

3.3.7.3. Rheology vs. structure of CS/surfactant complexes 90

$\begin{array}{ll}\text { CONCLUSIONS } & 91\end{array}$

$\begin{array}{ll}\text { REFERENCES } & 93\end{array}$ 


\section{INTRODUCTION}

Systems containing water, ionic or non-ionic surfactants and water-soluble polymer have been a subject of extensive investigations in the last ten years aimed at a fundamental understanding of their properties as well as at evaluation of their use in various technical applications.

A polymer that has been relatively little studied in spite of its technical importance, is cationic starch, CS. CS is widely used in paper processing as flocculation and retention aid. Cationic starch is a better retention aid than native starch, because due to the cellulose fibre/cationic starch attraction, it retains very effectively to the fibres and doesn't concentrate to the circulation water as badly as native starch. It is not only the fibres that are involved but also fines and fillers, which are much smaller particles than fibres. Their retention to the fibre network is extremely important. Fibres, fines and fillers are all negatively charged and therefore repel each other. Large cationic polyelectrolyte, cationic starch can adsorb on these negatively charged particles and collect them by formation bridges between them. In this way is its possible to effectively flocculate and also retain the fines and fillers. Optimal retention improves the wet strength of paper, and also important, the waste of these components decreases radically.

Starch has also been proved to improve the dry strength of paper, making the interfibre bonding stronger. It is added to the process between refiner and the headbox. Starch is also used as a size on uncoated papers. It improves the printability, prevents the dusting of paper and makes the paper surface smooth.

Cationic starches of high degree of substitution ( $>0.1)$ are also used in the paper coatings as well as in the headbox of paper machines, among other things in order to collect harmful anionic compounds from the water circle. This so-called "anionic trash" consists mainly of dissolved hemicelluloses, fatty and resin acids, and their salts, which are components of wood extracts. Cationic starch that is used as a retention aid can also produce, together with wood extracts very harmful precipitates, which can disturb the whole paper processing. The cationic starch-cellulose fibre interactions are extensively studied in the thesis of (van Steeg, 1992) but nothing has been published about interactions of cationic starch with surfactants. Thus, it is very important to get information about the interactions of these two substances. 
For example, interactions of surfactants with cationised cellulose, has been studied by (Goddard et al., 1976, 1977) and nonionic cellulose ethers have been subject of extensive studies by (Piculell, Lindman et al., 1992). The structure of starch is very similar to cellulose, but the difference on the bindings, which link the monoglucose units to form the polymer, makes their chemical behaviour very different. The polymer chains in starch are much more flexible than in cellulose, making the polymer more soluble in different solvents. The polymer chains of amylopectin are also branched, whereas cellulose has completely straight chain. Thus, actually starch is very different material to cellulose despite of their chemical similarity.

The aim of this is theses is to form better understanding about interactions between polyelectrolyte and oppositely charged surfactants: What are the parameters affecting these interactions and how can these systems be modelled. Another important part of this study was the determination of the structure of these complexes and how these structures might be modified.

Over the last few years, there has been an increasing interest in biodegradable materials. This interest was also a motivating factor in these studies, because chemicals used in these studies are nearly all derived from nature and also non-toxic and biodegradable. Starch is produced in huge amounts all around the world so it would be important to try to find new applications for its use instead of synthetic non-biodegradable polymers where possible.

\section{THEORETICAL BACKGROUND}

\subsection{Characteristic of polyelectrolytes}

Polyelectrolytes, such as cationic starch, have some special properties, which distinguished them from uncharged polymers. First, the mutual repulsion of their charges causes expansion of the chain far beyond the expansion from changes from poor to good solvents with ordinary polymers. The size of the polyelectrolyte random coil is, moreover, a function of the concentrations of polymer and added salt, since both influence the degree of ionisation. Secondly, the ionisation of the electrolyte groups leads to a variety of unusual effects in the presence of small amounts of salt. The intensity of the light scattering decreases because of the 
ordering of the molecules in solution, while osmotic pressure and ultracentrifugation behaviour are determined predominantly by the total charge on the molecule (Donnan effect). A parameter describing the charge of a polyelectrolyte is the reduced linear charge density, $\zeta$, which is related to the distance, $b$, between adjacent charges along the polymer backbone by the relation

$$
\zeta=e^{2} / 4 \pi \varepsilon b k T
$$

Properties depending on the size of the chain, such as viscosity and angular dependence of light scattering, are strongly affected by chain expansion.

The expansion of polyelectrolyte chains due to charge repulsion is most often described in terms of persistence length, $p_{e l}$. The formula for the electrostatic persistence length (Odijk ,1978, Schurr et al., 1986):

$$
P_{e l}=l_{B} /\left(4 \kappa^{2} b^{2} \xi^{2}\right)
$$

involves several factors. The quantity $l_{B}=e^{2} / k T$ is the Bjerrum length at which two electronic charges of magnitude $e$, in a solvent of dielectric constant , interact with energy $k T ; \kappa^{-1}$ is the Debye screening length, given by $\kappa^{2}=8 \pi l_{B} c_{s}$, with $\mathrm{c}_{\mathrm{s}}$ the concentration of monovalent salt in solution (modifications to multivalent salt are straightforward); $b$ is the distance between charges along the chain; $\xi$ is a number between 0 and $1\left(/ l_{B} / b\right)$ that accounts for a phenomenon known as counterion condensation. From this equation, it is clearly seen that the electrostatic persistence length decreases linearly with salt concentration and increases with the square of the charge density along the backbone.

Counterion condensation, advanced as a model and studied extensively by (Manning 1977-79) and others (Fixman, 1979 and Anderson et al., 1982), is a phenomenon that regulates the effective charge density on a polyelectrolyte chain. The maximum supportable charge density on the polyion is controlled by $\xi$. When the charge separations become less than $l_{B}$, counterions condense, or bind to the polyion, neutralising some of the charge and reducing the effective charge density to the critical value of $l_{B}$

\subsection{Surface-active agents}




\subsubsection{General}

Surfactants are characterised by the presence of two moieties in the same molecule, one polar and the other non-polar. The behaviour of surfactants in aqueous solution is determined by their tendency to move their hydrophobic part away from the solution and their hydrophilic part towards the solution. This dual tendency is responsible for adsorption of surfactants at interfaces and for the formation of such aggregates as micelles.

Surfactant molecules adsorb at the water/air interface with their hydrophobic groups away from the water and their hydrophilic groups in solution. A consequence of this is that some of the water molecules at the interface will be replaced by hydrocarbon or other non-polar groups. Since the interaction force between water molecules decreases, adsorption of surfactants at the interface results in a reduction in the surface tension of the solution.

\subsubsection{Micelle formation in aqueous solutions}

Above a certain concentration, the so-called critical micelle concentration ( $\mathrm{cmc})$, almost all of the added surfactant molecules are consumed in micelle formation, and the monomer concentration does not increase. Since only the surfactant monomers adsorb at the interface, the surface tension remains constant above the $\mathrm{cmc}$.

As mentioned above, surfactant molecules in aqueous solutions at concentrations above their cmc aggregate to form uniform sized structures referred to as micelles. Well-studied micelles like those of SDS have a near-spherical geometry over a wide range above the $\mathrm{cmc}$. Evidence also exists for the formation of cylindrical and other shapes in certain systems.

It has been found that surfactants with bulky hydrophilic groups and long, thin hydrophobic groups tend to form spherical/globular micelles in aqueous solutions. In contrast to this, surfactants with bulky hydrophobic groups and small hydrophilic groups tend to form lamellar or cylindrical micelles.

The shape of a micelle is related to its size. The size is normally expressed in terms of an aggregation number. Typical aggregation numbers obtained for commonly used surfactants varied from 30 to 90 . The size and aggregation number of surfactants change markedly with such variables as ionic strength and temperature. 
2.2.3 Factors affecting the $c m c$ and aggregation number

An increase in the length of the hydrocarbon chain decreases the $c m c$. Linear hydrocarbon surfactants tend to have much lower $\mathrm{cmc}$ than their branched-chain counterparts. The presence of a double bond in the chain increases the $\mathrm{cmc}$.

Hydrophilic group. In aqueous media, ionic surfactants have a much higher $c m c$ than nonionic surfactants with a corresponding hydrocarbon chain length. Correspondingly, as the number of ionising groups increases in a surfactant molecule the $\mathrm{cmc}$ increases. As the position of the ionic group changes from the terminal position to more central positions, the $\mathrm{cmc}$ increases.

Counterions adsorb at the ionic micelle surface and stabilise it; the extent of adsorption is dependent on the micelle charge density. The higher the adsorption of counterions, the lower the $c m c$. An increase in the valency of counterion decreases the $c m c$. An increase in hydrated radius, on the other hand, increases the $\mathrm{cmc}$.

Electrolytes have a significant effect on the $c m c$ of ionic surfactants (Corrin et al. 1947). An increase in the concentration of the electrolytes progressively contracts the electrical double layer around the micelle and in the process stabilizes the micelle. This is manifested as a reduction in $c m c$ with electrolyte concentration. Note, however, that an increase in the electrolyte level need not increase the amount of counterions bound in the Stern layer of the micelle (Lindman, 1984).

The process of micellisation itself is dependent on temperature in a complex way. For example, cooling of a micellar solution of SDS below $12{ }^{\circ} \mathrm{C}$, results in the precipitation of the surfactant. Here the concentration of the surfactant is equal to the $\mathrm{cmc}$. This temperature, referred to as the Krafft temperature or Krafft point, is the temperature at which the solubility of the surfactant equals the $c m c$, and above this temperature, the total solubility of the surfactant increases markedly because of the formation of micelles. Below this temperature, only surfactant monomers exist in solution and, therefore, the total solubility is drastically limited. The Krafft point of surfactants depends upon the chain length of the hydrophobe as 
well as the type, valency, and concentration of counterions. The Krafft point, in general, increases with alkyl chain length.

\subsubsection{Mixed micellisation}

In solutions containing mixtures of surfactants, the tendency to form aggregated structures can be substantially different from that in solutions containing only the pure surfactants. The tendency for components to distribute between a non-aggregated state and an aggregate may vary from component to component for mixtures. Therefore, the surfactant composition of a micelle may differ greatly from the composition of the non-aggregated surfactants.

\subsubsection{Modelling mixed surfactant systems}

In models of mixed micellisation, the Gibbs energy of mixed micelle formation is discussed in terms of several contributions.

1) Contact energy of the surfactant. When the hydrophobic moiety of the surfactant is transferred from aqueous environment into the liquid hydrocarbon-like interior core of the micelle, there is a favourable change in the contact energy, which depends on the hydrocarbon chain length but is mainly due to the creation of water/water contacts.

2) Conformational entropy. The transfer of the hydrophobic moiety of the surfactant into the micelles also implies an energetically favourable change in their conformational energy.

3) Aggregate core-water interfacial Gibbs energy. If the hydrophilic moiety is not very bulky, the formation of a micelle generates an interface between the hydrophobic core region and the surrounding aqueous solution.

4) Head group steric interactions. In the formation of micelles, the polar head groups of the surfactant molecules are crowded onto the aggregate surface. This generates steric repulsions between the head groups.

5) Head group ionic and dipole interactions. For ionic surfactants, repulsive electrostatic interactions arise at the micellar surface. For zwitterionic surfactants, one has to consider the interactions between the permanent dipoles of polar head groups. 
6) Gibbs energy of mixing of surfactant tails. This contribution accounts for the entropy and enthalpy of mixing of the surfactant tails of molecules $A$ and $B$ in the hydrophobic core of the micelle.

In the simplest approach, it is assumed that the only contribution to the Gibbs energy of micellisation from the mixing process is the entropy of mixing of surfactant tails. This ideal mixing model leads to a simple equation for the $c m c$ of $n$ different surfactants:

$$
\frac{1}{C_{m i x}}=\sum_{i=1}^{n} \frac{\alpha_{i}}{f_{i} C_{i}}
$$

where $C_{m i x}$ is the $c m c$ of the surfactant mixture, $a_{i}$ is the fraction of surfactant $i$ of the total amount of surfactant, $f_{i}$ is its activity coefficient and $C_{i}$ its $c m c$. In ideal mixed micelles, the activity coefficient $f_{i}$ is unity and so the surfactants do not interact with each other. Although few micellar systems behave ideally, this model is useful as a reference system in the description of reasons for non-idealities.

\section{2. 4. 2. Synergism in mixing of different surfactants}

Mixtures of different surfactant types often exhibit synergism in the formation of micelles, i.e. deviations from ideal mixing results in substantially lower $c m c$ s and interfacial tensions than would be expected based on the properties of the unmixed surfactants alone.

The most commonly used models of non-ideal mixing are based on the regular solution approximation. This assumes that the excess Gibbs energy of mixing consists only of an enthalpy term, while there are no excess entropy contributions. For a binary mixture the excess enthalpy of mixing per mole at the $c m c$ is defined by

$$
H^{E}=\beta x_{1}\left(1-x_{1}\right) R T=G^{E}
$$

where the parameter represents the molecular interaction between two surfactants.

In this model, the micellar mole fractions of a binary surfactant mixture can be calculated by the iterative solution of the following expression: 


$$
\frac{\alpha_{1} C_{2}^{*} f_{2}\left(1-x_{1}\right)}{\alpha_{2} C_{1}^{*} f_{1} x_{1}}=1
$$

where $C_{1}{ }^{*}$ and $C_{2}{ }^{*}$ are the $c m c$ s of the pure component and $x_{1}$ is the mole fraction of component 1 in the mixed micelle. The non-ideality is characterised by the net interaction parameter, which is related to the activity coefficients by:

$$
\begin{aligned}
& f_{1}=\exp \left\{\beta\left(1-x_{1}\right)^{2}\right\} \\
& f_{2}=\exp \left\{\beta\left(x_{1}\right)^{2}\right\}
\end{aligned}
$$

From the regular solution theory, it follows that in a binary surfactant system, can be calculated from $c m c$ s of the surfactant mixture and the $c m c$ s of the pure components according to:

$$
\beta=\frac{\ln \left[\alpha_{1} C_{M i x}^{*} /\left(x_{1} C_{1}^{*}\right)\right]}{\left(1-x_{1}\right)^{2}}=\frac{\ln \left[\alpha_{2} C_{M i x}^{*} /\left(\left(1-x_{1}\right) C_{2}^{*}\right)\right]}{x_{1}^{2}}
$$

where $C^{*}{ }_{m i x}$ is the $c m c$ of the surfactant mixture.

\subsection{Polymer-surfactant interactions}

\subsubsection{Driving forces}

The interactions responsible for association phenomena in polymer-surfactant systems are mainly:

1. Hydrophobic interactions between polymer and surfactant molecules. This kind of interaction will be particularly important for hydrophobically modified polymers.

2. Hydrophobic interactions between hydrophobic parts of surfactant molecules. 
3. Hydrophobic interactions between polymer molecules.

4. Electrostatic interactions between polymer molecules.

5. Electrostatic interactions between polymer and surfactant molecules; they may be repulsive or attractive, depending on whether the molecules have similar or opposite charges. 6. Electrostatic interactions between hydrophilic parts of surfactant molecules. These repulsive interactions are strongly unfavourable for surfactant micellisation.

For the different interactions mentioned, the main driving force of association in polymersurfactant systems in general comes from the hydrophobic interactions between surfactant molecules. For hydrophobic polymers, the hydrophobic interaction between polymer and surfactant alkyl chains, will contribute more or less strongly, depending on the system. Because of delicate energy balances, even quite small contributions from the other interactions can have dramatic influences on the self-assembly and induce important surfactant-polymer interactions. Ionic surfactant micellisation will, because of the electrostatic interactions, be particularly susceptible to changes.

There is obviously an interrelation between specificity and cooperativity such, that for a higher degree of specificity, cooperativity will be less important and smaller aggregates may form. The variation in driving forces will have its counterpart in the structural description. For many polyelectrolyte-ionic surfactant systems, there will be just a long-range effect on micellisation, and the polymers chains and the surfactant micelles may remain at some distance.

\subsubsection{Binding isotherms}

A characterisation of the surfactant binding to the polymer (at fixed polymer concentration) is to represented as a "binding isotherm" (Fig. 1). 


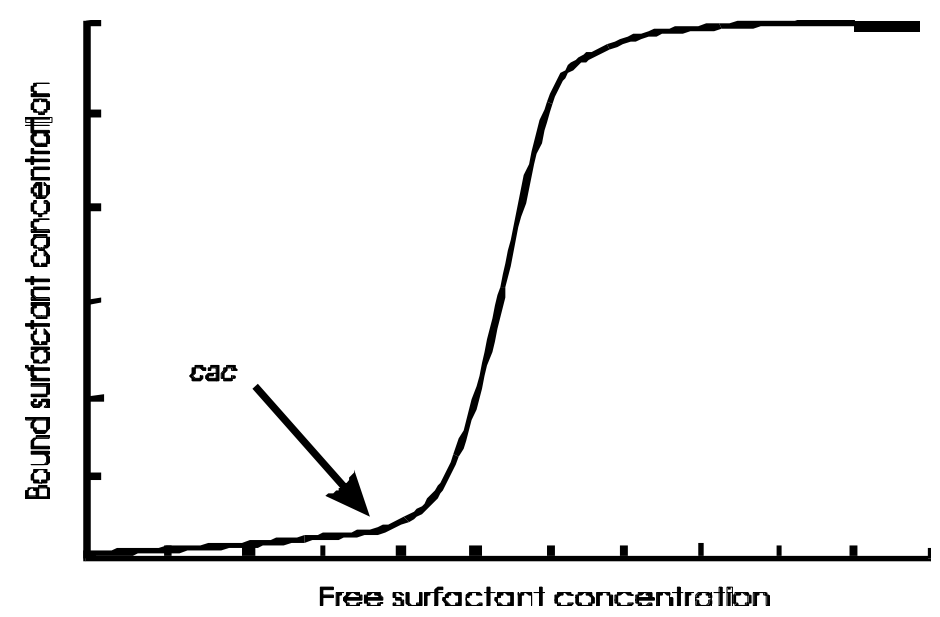

Figure 1. A typical binding isotherm for the binding of a surfactant to a polymer. The critical aggregation concentration, cac, is indicated.

The isotherm has a marked sigmoid shape, which is an indication of cooperative binding, and the onset of the surfactant binding often occurs at a certain, rather well defined, surfactant concentration. This concentration is called the critical aggregation (or association) concentration (cac). This notion, which was introduced by (Chu and Thomas, 1986), indicates that the surfactant molecules form aggregates upon interacting with the polymer chains. The levelling out of the binding isotherm at higher surfactant concentrations is due to saturation of the polymer with surfactant, and indicates the maximum amount of surfactant that can be bound per polymer unit. For many systems, this level is not reached due to phase separation, or is obscured by the formation of free micelles.

\subsubsection{Strength of interaction}

A description in terms of a $c a c$ value clearly presupposes a driving force similar to that of normal surfactant micellisation and a strongly cooperative binding. The free energy of micellisation can be written

$$
\Delta G_{m}^{o}=R T \ln c m c
$$

and the free energy of surfactant binding to polymer is analogously expressed as:

$$
\Delta G_{b}^{o}=R T \ln c a c
$$


Therefore, we can derive the free energy per mole surfactant for the reaction: free micelle $\int$ polymer-bound micelle, as

$$
\text { ) } G_{P S}^{o}=R T \ln (\mathrm{cac} / \mathrm{cmc})
$$

This quantity is a convenient measure of the strength of the interaction between the surfactant and the polymer. In particular, it is impossible to have a cac that is higher than the $c m c$ of the surfactant; in that case, the surfactant molecules would prefer to form free micelles instead of polymer-bound ones, and surfactant binding to the polymer would not occur. If the polymer preferentially interacts with a micelle, the free energy of the micelle, and thus the $\mathrm{cmc}$, should be lowered. On the other hand, while a lowering of the $c m c$ demonstrates an interaction, it is not necessarily an indication of a complex formation between the polymer and the surfactant. For example, addition of salt also reduces the $c m c$ and addition of polyelectrolyte of the same charge as the surfactant has a similar effect.

The strength of the interaction varies considerably between the different types of polymersurfactant systems. In particular, the interaction between a polyelectrolyte and an oppositely charged surfactant is strongly favoured, and a decrease of the cac relative to the $\mathrm{cmc}$ by several orders of magnitude is commonly observed.

\subsubsection{Interactions between polyelectrolytes and oppositely charged surfactants}

Systems of a polyelectrolyte and an oppositely charged surfactant in dilute solution have been extensively studied. Due to the strong attraction between two species, the interaction starts at very low surfactant concentrations and is difficult to study by most conventional techniques. Often, binding of an ionic surfactant to polyions starts at a concentration, which is several magnitudes lower than the $c m c$ in polymer-free solution. The surfactant binding is also highly cooperative in these systems, pointing to the contribution from interactions among the adsorbed surfactant molecules, and the formation of polymer-adsorbed micelles or micelle-like clusters.

There are some general features of relevance for systems of polyelectrolyte and oppositely charged surfactant. First, it should be noted that, due to general electrostatic interactions, polyelectrolytes give rise to an uneven distribution of ions in a solution. The concentration of 
counterions is strongly enhanced close to the polyelectrolyte and decays rapidly with an increased distance from it. The uneven distribution of counterions also applies to monomeric surfactant counterions, present in the polyelectrolyte solution. Thus, the concentration of surfactant ions is enhanced close to the polyelectrolyte in systems of polyelectrolyte and oppositely charged surfactant. The major reason for cooperative binding of surfactant molecules to an oppositely charged polyelectrolyte is the electrostatic stabilization of the surfactant micelles. For hydrophilic polyelectrolytes, such as polysaccharides, there is no driving force to make the polymer segments to penetrate into the hydrophobic interior of the micelle. The situation is thus very different from systems of an uncharged polymer, where forces other than pure electrostatic ones play a major role. The situation will be altered if the polyelectrolyte also contains hydrophobic moieties.

\subsubsection{Influence of the surfactant}

The length of the hydrocarbon tail of the surfactant is a crucial parameter for the interaction with a polyelectrolyte. An increasing difference between $\log (\mathrm{cmc})$ and $\log (\mathrm{cac})$ when surfactant chain length is increased indicates that the interaction between polymer and surfactant is increasing (Figure 2).

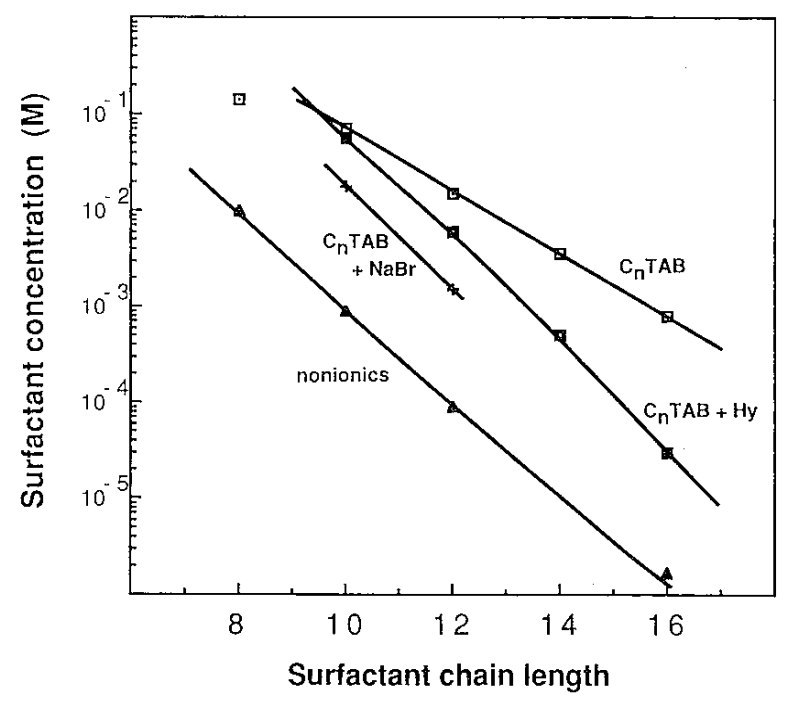

Figure 2. Cac values for $C_{n} T A B$ and $H y$ at $25^{\circ} C$ compared with the cmc values of the surfactant. Also, included are cmc values for these surfactants in the presence of $0.5 \mathrm{M} \mathrm{NaBr}$, and $\mathrm{cmc}$ values for non-ionic surfactants of the $C_{n}(E O)_{6}$ type. Ref. from (Thalberg and Lindman, 1989) 
This can be attributed to the uneven distribution of counterions between the bulk and the micellar surface, which is unfavourable for the formation of normal micelles and which is more pronounced for a longer surfactant; a lower $c m c$ gives a lower intermicellar concentration. The binding of an oppositely charged polyelectrolyte to the micelles entails a release of the ordinary counterions, and, therefore, leads to a larger increase in the entropy of the counterions for a surfactant of longer chain length (Thalberg and Lindman, 1989). For surfactants with less than a certain chain length (10 for the Hy- $\mathrm{C}_{n} \mathrm{TAB}$ system), there will be no binding to the polyelectrolyte. In this case, normal free micelles are favoured relative to the polyelectrolyte-bound ones.

A second way of conceiving the interaction is to consider the formation of polyelectrolytesurfactant aggregates with a relatively low charge. The surfactant chain length dependence for the formation of these aggregates will therefore be similar to the alkyl chain length dependence for the formation of non-ionic micelles.

\subsubsection{Influence of the polyelectrolyte}

The properties of the polyelectrolyte are also of major importance for the interaction with surfactants. One important parameter is the reduced linear charge density of the polyelectrolyte, . (Eq. 1). Cac data for different systems as a function of . are given in Figure 3. The trend is clearly that an increase in linear charge density gives rise to a stronger interaction. Apparently, other forces also play a role besides the pure electrostatic ones, and modulate the interaction between the polyelectrolyte and the surfactant micelles. Such contributions may arise from different types of charged groups or from the presence of hydrophobic moieties on the polyelectrolyte. In addition, the flexibility of the polymer backbone and the type of counterions present may influence the interaction. 


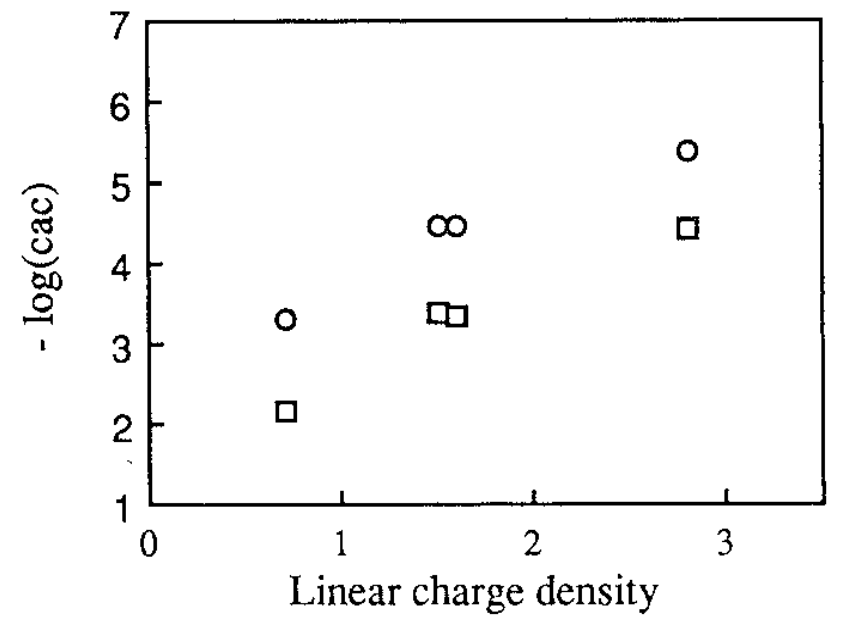

Figure 3. $\log (\mathrm{cac})$ for $C_{12} T A B(\mathrm{R})$ and $C_{14} T A B(\mathrm{~N})$, polyelectrolytes varying the reduced linear charge density, .. The polyelectrolytes are PA (polyacrylate) $(.=2.8)$, alginate $(.=1.5)$, and pectate (. = 0.6). Data is from (Hayakawa et al., 1991 and Thalberg et al., 1989).

The cooperativity in surfactant binding is also influenced by the charge density of the polyelectrolyte; the cooperativeness decreases rapidly with a reduced charge density of the polyelectrolyte, but a marked cooperativeness is also observed for the binding of $\mathrm{C}_{10} \mathrm{TAB}$ to Hy, which has a relatively low charge density (Thalberg and Lindman, 1989, Thalberg et al., 1990a, 1991a). Thus, the cooperativeness also depends on other features of the polyelectrolyte, such as the flexibility of the chains and hydrophobic character of the repeating units.

\subsubsection{Effect of salt}

The effect of salt on the polyelectrolyte-surfactant complexes is opposite to the influence of salt in micellar systems, where stabilization occurs manifested by a lowering of the $\mathrm{cmc}$. The effect of salt is twofold: 1) A reduction in the electrostatic interaction between polyelectrolyte and the surfactant, and 2) The stabilization of the surfactant aggregates. The first mechanism will dominate at low ionic strength, while at high ionic strength, the second mechanism will take over. A decrease in $c a c$ at high-salt concentrations, similar to the $c m c$ behaviour, can therefore be expected.

\section{4. Polyelectrolyte/surfactant complexes}

2. 4. 1. Phase behaviour of hydrophilic polymers with pure surfactant micelles

Hydrophilic non-ionic polymers are well soluble in water. There are no hydrophobic interactions between these polymers and surfactants. In most cases, the phase separation is 
segregative, but if the polymer and the surfactants are oppositely charged, associative phase separation of the complexes may occur. Adding a certain amount of salt will suppress the association and eventually lead to a segregative phase separation.

\section{4. 1. 1. Oppositely charged mixtures}

High-charged polymers differ strongly from the corresponding non-ionic species. There is a strong enrichment of counterions close to the polyelectrolyte and this uneven counterion distribution lowers the entropy markedly. For ionic surfactants, this entropic effect strongly counteracts micellisation. This is the major reason for the much higher $c m c$ of ionic surfactants compared to non-ionics (Evans et al., 1994). In the presence of electrolytes, micelles are stabilised, the higher is the valency of the oppositely charged ion. This polyelectrolyte induced lowering of the $c m c$ of oppositely charged surfactants is also referred to as a cooperative binding of the surfactant to the polyelectrolyte.

Another observation is that in oppositely charged mixtures associative phase separation occurs at quite low surfactant concentrations, often only slightly above the cac (Goddard (1986a, b and Goddard and Ananthapadamabhan, 1993). The concentrated phase could be a solid or a highly viscous gel-like solution. Addition of large amount of salt inhibits the phase separation and a redissolution of the complex phase takes place when an excess of surfactant is added.

The phase behaviour of oppositely charged surfactant and hydrophilic polyelectrolyte depends on the choices of polyelectrolyte, surfactant and simple salt. The minimum concentration of surfactant required for phase separation decreases strongly as its chain length increases, i.e. as cmc decreases. This observation supports the notion that associative phase separation involves the polyion and the self-associated surfactant. The redissolution concentration is less dependent on the surfactant chain length. An increase in the simple salt concentration leads to shrinkage of the two-phase area. The amount of salt needed to give redissolution, the critical electrolyte concentration (cec), increases with increasing surfactant chain length and is strongly dependent on the surfactant concentration. $\mathrm{Cec}$ also depends on the nature of the added salt, the anion in particular. Increasing the valency of a simple ion lowers counterion entropy effects and decreases the entropic gain of polyion-micelle association. This reduces the 
tendency to associative phase separation. The salt effects have been confirmed for mixtures of cationics with $\mathrm{NaPa}$ (sodium poly(acrylate)) by (Hansson and Almgren, 1994). They suggested that the increasing $c e c$ with increasing surfactant chain length could be attributed to a larger aggregation number of micelles. Tendency to association increases strongly with increasing charge density of the polyelectrolyte. This is nicely shown in a study of poly(acrylate-coacrylamide) (Ranganathan and Kwak, 1996) where the ionisation of the polymer was varied. The experiment of varying the charge density of the micelles by mixing in non-ionic surfactant has also been made ( $\mathrm{Li}$ and Dubin, 1995). As expected, reducing the micellar charge density or the charge density of the polymer markedly reduces the extent of the phase separation.

\section{4. 2. Structure of complexes}

\subsubsection{Conformation of surfactant molecules}

Polyelectrolyte/oppositely charged systems have been widely studied and especially the aggregation numbers of the associating surfactants have been determined. The general picture emerging from these studies is that micelle-like surfactant clusters bind to the polymer chains so that the charged segments are neutralised by the oppositely charged head groups of the surfactant. The majority of the investigations directed towards the structure of polyelectrolytesurfactant complexes have focussed on the properties of the surfactant aggregates; notably their aggregation numbers.

In particular, the use of fluorescence techniques has contributed to the establishment of this structure, but also different NMR techniques have been used. The first report of aggregation numbers in polyeletrolyte/surfactant system is of (Abuin and Scaiano, 1984). They studied the system of PSS and $\mathrm{C}_{12} \mathrm{TAB}$ and found that the surfactant forms small clusters of only up to 10 monomers absorbed into the polymer chains. (Zhen and Tung, 1992) studied extensively interactions of NaCMA (sodium carboxymethylamylose) with SDS. The cac was found to be $3 * 10^{-3} \mathrm{M}$ for SDS. Dynamic fluorescence-quenching measurements indicated that the aggregation number of associated micelles is smaller than for free micelles. $\left(\mathrm{N}_{\mathrm{agg}} \sim 47\right.$; $\left.\mathrm{NaCMA}=1.6 \mathrm{w} \%,[\mathrm{SDS}]=5^{*} 10^{-3} \mathrm{M}\right)$. For $\mathrm{C}_{16} \mathrm{TAB}$ the $\mathrm{N}_{\mathrm{agg}}$ ranges from 36 to 55 , as compared 147 for the free micelles. There have been many studies aimed at the determination of surfactant aggregation numbers in polyelectrolyte/surfactant systems. The results from these numerous studies can be summarised as follows. 
$\mathrm{N}_{\mathrm{agg}}$ has been found to be independent of the concentration of the bound surfactant, $\mathrm{C}_{\mathrm{b}}$ (Hansson and Almgren, 1994,1995). This behaviour has been illustrated by the studies of (Anthony and Zana, 1996). They studied binding of $\mathrm{C}_{12}$ TAC to PS1 (poly(maleic acid-comethyl vinyl ether) and PS4 (poly(maleic acid-co-butyl vinyl ether). The constancy of $\mathrm{N}_{\mathrm{agg}}$ indicates that the surfactant binding to polyions results in the formation of aggregates of constant composition and, thus, involving a constant length of polyion, in number increasing with $\mathrm{C}_{\mathrm{b}}$.

In the same studies was also found that $\mathrm{N}_{\mathrm{agg}}$ increases with " (degree of neutralization) for PS4 but decreases for PS1. This difference has been attributed to the fact that the side methyl groups of PS1 are short and contribute very little to the formation of the mixed aggregates. On the contrary, with, PS4, the side butyl chains interact hydrophobically with the surfactant alkyl chains and the aggregates are similar to mixed micelles of two surfactants. The latter form only small micelles and its mixed micellisation with a longer chain surfactant results in micelles of aggregation number smaller than for the longer surfactant. (Kiefer et al., 1992) also observed an increase of $\mathrm{N}_{\mathrm{agg}}$ with " in the system of PAA/C $\mathrm{C}_{14} \mathrm{TAB}$ in the presence of $10 \mathrm{mM} \mathrm{KBr}$.

The $\mathrm{N}_{\text {agg }}$ values for polyelectrolyte-bound aggregates increase with the surfactant chain length as in polymer-free systems (Almgren et al, 1992), but decrease with temperature (Hansson and Almgren, 1995) and are independent of the nature of the surfactant counterion (Hansson and Almgren, 1994) and of the added electrolyte (Hansson and Almgren, 1995). Thus, there is series of results in contrast with the behaviour of polymer-free surfactant solutions.

The bound aggregates have been shown to be relatively polydisperse with $F / N_{\text {agg }}$ values ranging between 0.3 and $0.7(\mathrm{~F}=$ standard deviation of the distribution) (Almgren et al. 1992). Almgren et al. have performed extensive determinations of $\mathrm{N}_{\mathrm{agg}}$ for variety of systems (NaPSS/C $\mathrm{C}_{\mathrm{n}} \mathrm{TAB}$ and $\mathrm{C}_{\mathrm{n}} \mathrm{TAC}$ (Almgren et al., 1992); NaPAA or NaPSS and $\mathrm{C}_{\mathrm{n}} \mathrm{TAB}$ (Hansson and Almgren, 1994); NaPVS or NaDXS and $\mathrm{C}_{12}$ TAB (Hansson and Almgren, 1995); NaHy and $\mathrm{C}_{10} \mathrm{TAB}$ or $\mathrm{C}_{12} \mathrm{TAB}$ (Thalberg et al., $1991 \mathrm{a}, \mathrm{d}$ ). The $\mathrm{N}_{\text {agg }}$ values were found to be close to those for polymer-free aggregates for PS1("=1)/C 12 TAC, NaPAA/C 12 TAB, NaHy/C 12 TAB and NA-CMA (sodium carboxyamylose)/C 16 TAB (Zhen and Tung, 1992). These polyelectrolytes are all highly hydrophilic. On the other hand, the $\mathrm{N}_{\mathrm{agg}}$ values in the PS4("=1)/ $\mathrm{C}_{12}$ TAC and NaPSS/ $\mathrm{C}_{12} \mathrm{TAB}$ or $\mathrm{C}_{12} \mathrm{TAC}$ were found to be smaller than those in the 
absence of polyelectrolyte. Both PS4("=1) and NaPSS behave like hydrophilic polyelectrolytes. Nevertheless, they show an effect of hydrophobicity arising from the presence of butyl chains and phenylene groups. A partial penetration of the polymer chains in the micelles result in lower $\mathrm{N}_{\text {agg }}$ values. Finally, the $\mathrm{N}_{\text {agg }}$ values in the $\mathrm{NaPVS} / \mathrm{C}_{12} \mathrm{TAB}$ and $\mathrm{NaDXS} / \mathrm{C}_{12} \mathrm{TAB}$ systems were found to be larges than for pure $\mathrm{C}_{12} \mathrm{TAB}$ micelles. This was interpreted as due to the special nature of sulphate group (Hansson and Almgren, 1995), as similarly the nature of counterion can affect micelle aggregation number.

In some polyelectrolyte/surfactant systems, such as $\mathrm{NaPAA} / \mathrm{C}_{12} \mathrm{TAB}$ or $\mathrm{C}_{12} \mathrm{TAC}$ systems, phase separation upon addition of salt yielded two liquid phases: dilute phase and a concentrated one (Hansson and Almren, 1994). The values of $\mathrm{N}_{\mathrm{agg}}$ in the two phases differ only slightly. The difference can be up to $15 \%$.

\section{4. 2. 2. Conformation of the polyelectrolyte}

Information of this topic is traditionally mainly obtained from viscosity measurements on dilute polymer solutions. Recently scattering studies with SANS and SAXS have provided further information. A decrease in the dimensions of the polyelectrolyte coils was observed for $\mathrm{NaPA}-\mathrm{C}_{\mathrm{n}} \mathrm{TAB}$ solutions (Thalberg et al., $1991 \mathrm{c}$ ). Certainly, coiling of the chains can be expected both for electrostatic reasons, as polyelectrolyte chains in pure water are extended, and for topological reasons, i.e., in order to create a large contact area between polymer chain and the micelle. (Herslöf and Sundelöf, 1992) studied the viscosity in dilute solution for $\mathrm{NaHy}-\mathrm{C}_{16} \mathrm{TAB}$ system after addition of salt $(\mathrm{NaBr})$. A reduction in the viscosity was found as compared to the surfactant-free system, but only below a certain salt concentration. Apparently, the polyelectrolyte-surfactant interaction is fully screened out at high salt concentration. Below this concentration, the viscosity decreased rapidly with a reduced salt concentration until the phase separation limit was reached.

Recently, Monte Carlo simulations have been applied to investigate complex formation between micelles and hydrophilic polyelectrolytes (Wallin and Linse, 1995). The scope of these studies was to study how different system parameters as flexibility and linear charge density of the polymer, and the chain length of the surfactant affect the cac/cmc ratio. The conformation of polyelectrolyte-surfactant complexes was found to depend on the flexibility of the polymer chains. A flexible polyelectrolyte chain can easily wrap around the micelle and the 
micellar charge is neutralised by polyelectrolyte charges located close to the micelle, contributing to lower $c a c$ values. However, when the bare persistence length is of the order of the micellar dimension, the polyelectrolyte is wrapped less tightly around the micelle and obviously, the possibility of the polymer to replacing small counterions of the micelle is diminished. However, the results with relatively stiff CS show that this is not a general rule.

\section{4. 3. Native starch-surfactant complexes}

It is very well known that amylose exists in three different helical conformations in aqueous solutions. These polymorphs are named A-, B- and V-forms. The A- and B- forms comprise parallel-packed, left-handed double helices. The A- and B-forms can be considered as extended helices with, unlike the V-form, no hydrogen bonding between consecutive turns of the helices. The V-form occurs only in the presence of some complex-forming ligand. It has for a long time been known that amylose forms helical inclusion complexes with a variety of organic compounds such as lipids (Snape et al., 1998), carboxyl acids (Codet et al., 1993) and different surfactants (Yamamoto et al., 1983). The helical coil formed by the amylose/lipid inclusion complex in aqueous solution has been demonstrated to comprise from 6 to 8 repeating glucose units per helical turn. Amylose takes so-called V-form conformation when forming the inclusion complexes. The $\mathrm{V}$ amylose is a generic name for crystalline amyloses, obtained as single helices co-crystallized with compounds such as water, iodine, DMSO, alcohols or fatty acids. In the V-form, a single chain of amylose forms a helix with a relatively large cavity. The central cavities of the V-forms have a pitch of about $8 \AA$ per turn. A detailed X-ray conformational analysis of the hydrated helix with a guest molecule inside has been reported by (Rappenecker et al., 1981). (Yamamoto et al., 1983) found that the binding of sodium alkyl sulphates to amylose is cooperative when the DP of polymer is big enough, and that its cooperativeness depends on the carbon chain length of the surfactant. Also, the outer branches of the amylopectin molecule are assumed to form the same kind of inclusion complexes with suitable lipids (Kubik et al, 1995, Thomasik et al., 1995, Eliasson et al., 1995).

2. 4. 4. Polyelectrolyte-surfactant complexes with high range order

2. 4. 4. 1. In organic solvents and in the solid state 
Polyelectrolyte-surfactant complexes have known for some years to form ordered complexes in organic solvents and in the solid state. For a review, see (Ober and Wegner, 1997). (Antonietti et al., 1994) have observed that complexes that precipitate out when aqueous solution of NaPSS and alkyltrimethylammonium bromides are mixed together can be redissolved in organic solvents such as dimethylformamide, tetrahydrofuran or ethanol, where they behave like polyelectrolytes. Cast films of some of these complexes showed highly ordered mesophases of the lamellar type (Antonietti et al., 1995). Such structures appear to be of a general character since they were observed with several polyion/surfactant ion complexes: copolymers of 2-acrylamido-2-methyl-1-propanesulphonic acid with N-octadecylacrylamide, at different ratios of the two monomers with $\mathrm{C}_{16} \mathrm{TAB}$ (Antonietti et al. 1996), and poly(dimethyldiallylammonium chloride)/natural lipid (Antonietti et al. 1998).

(Kabanov et al., 1994, 1995) investigated complexes of polycations and polyanions with the anionic surfactant AOT (sodium diethylhexylsulfosuccinate) in organic solvents in the presence of water. The complexation of polyelectrolytes of the ionomer type (partially sulfonated polystyrene) by AOT in m-xylene resulted in a partial disruption of the aggregates formed by the ionomer (Kabanov et al., 1994). Poly(N-ethyl-4-vinylpyridinium bromide) was found to be soluble in octane and hexane in the presence of AOT (Kabanov et al., 1995) and in the presence of water pools formed by AOT, where it adopted a rather compact conformation (Kabanov et al., 1994). At low water content, the authors proposed a comb-like model for the complex.

\section{4. 4. 2. In aqueous solutions}

In the first reported study, as expected, (Harada and Nozakura, 1984) described electron microscope (TEM) studies of polyelectrolyte complexes made from poly(vinyl sulphate), PVS, with $\mathrm{C}_{16} \mathrm{TAB}$. By mixing dilute aqueous solutions of the potassium salt of PVS with $\mathrm{C}_{16} \mathrm{TAB}$, precipitates were formed which showed maximum turbidity when a 1:1 mixture was prepared. The precipitates possessed complex lamellar structures that were measured by X-ray diffraction and found to be of thickness 50-60 $\AA$, the length of two surfactant molecules. Similar layered structures were reported for the complex made from ionene-3,4 and SDS. 
Osada et al. made extensive studies of $\mathrm{x}, \mathrm{y}$-ionene/SDS and PVS or PAMPS (poly[2(acrylamido)-2-methylpropanesulphonic acid]/n-alkylpyridinium surfactants systems (Chen et al., 1998 and Kim et al., 1999). The results showed that n-alkylpyridinium surfactants form lamellar mesophases in the complexes of NaPSS, but hexagonal close-packed of spherical micelles with PAMPS. They assumed that the reason for these different structures might be the more rigid chains of NaPSS, which may inhibit the folding of polymer chains around the micelles.

Kabanov et al. studied the NaPA-gel/alkyltrimethylammonium bromide complexes (Khandurina et al., $1994 \mathrm{a}, \mathrm{b}, \mathrm{c}$ ). The complexes were found to have lamellar structure when the chain length of the surfactant was long enough (>14). The NaPA complexes with $\mathrm{C}_{14} \mathrm{TAB}$ and $\mathrm{C}_{12} \mathrm{TAB}$ were amorphous without any long-range order. Benjamin $\mathrm{Chu}$ and his co-workers have extensively studied ordered polyelectrolyte/surfactant structures. They studied poly(diallylmethylammonium chloride, PDADMAC) gels with different sodium alkyl sulphates (Sokolov et al., 1996, 1998) and found that these complexes have structure of hexagonal close-packed of cylindrical micelles with SDS, STS, and SHS. The SDS complex was found to have a cubic structure. The structure was dependent on surfactant chain length and temperature, thus SOS or SHS complexes do not have any ordered structure at room temperature, but at -5EC, PDADMAC/SOS the complex has a cubic structure. Recent studies (Zhou et al., 1999) on the complexes of PMAA and (PMAA/NIPAM) systems showed a clear dependence of the complex structure formed on chain length of the surfactant. With surfactants of different chain lengths, PAA forms different structures $\left(\mathrm{C}_{8} \mathrm{TAB}\right.$, micellar; $\mathrm{C}_{10} \mathrm{TAB}$, $\mathrm{C}_{12} \mathrm{TAB}, \mathrm{C}_{14} \mathrm{TAB}$ and $\mathrm{C}_{16} \mathrm{TAB}$ cubic Pm3n). PMAA/NIPAM-complexes are with: $\mathrm{C}_{18} \mathrm{TAB}$ cubic, $\mathrm{C}_{14} \mathrm{TAB}$ and $\mathrm{C}_{16} \mathrm{TAB}$ face-centred cubic close packing of spheres, $\mathrm{C}_{12} \mathrm{TAB}$ hexagonal close packing of spheres. PMAA/NIPAM is more hydrophobic than PMAA, and the hydrophobicity has a clear effect on the structure of polyelectrolyte/surfactant complexes. In the same studies, PSS/surfactant complexes were also compared with PMAA/surfactant complexes. PSS has a more rigid and stiffer chain than PMAA. The structure of PSS/C 14 ${ }_{18} \mathrm{TAB}$ complexes was a 2D hexagonal close packing of cylinders. Thus, the structure of PSS and PMAA complexes is quite different. It was assumed that this difference is due to the rigid chain of PSS that makes it difficult to wrap the polymer chain around spherical micelles. 
Very recently (Ilekti et al., 1999) published studies about $\mathrm{NaPA} / \mathrm{C}_{16} \mathrm{TAB}$ system. They produced a phase diagram that shows the structure of $\mathrm{NaPA} / \mathrm{C}_{16} \mathrm{TAB}$ complexes as a function of NaPA and $\mathrm{C}_{16} \mathrm{TAB}$ concentrations. The structure of the complex was found to chance with increasing concentration of complex phase from micellar to cubic and hexagonal. At higher temperatures and at high concentrations lamellar structures can also formed. These phase transitions were also compared with the phase transitions of pure surfactant in the same conditions, and it was found that the existence of polyelectrolyte can lead to different structures.

There are also some studies about the effect of adding polyelectrolyte to the liquid crystalline phase of some surfactants. (Kosmella et al., 1996 and Ruppelt et al., 1997) found that the intercalation of sodium polyacrylate into mesophases formed by alkyltrimethylammonium bromides induced some disordering, but did not substantially affect the phase structures.

These studies can be summarised as follows: 1) Alkyl chain length of the surfactant affects the formation and transition of highly ordered structures inside polyelectrolyte/surfactant complexes. The longer the chain length, the stronger are the hydrophobic interactions between surfactant and polymer chains and the smaller the volume ratio of polar-to-apolar moieties of surfactant, both of which determine the self-assembly behaviour of the surfactant inside the complexes. 2) Both electrostatic and hydrophobic interactions are very important for inducing the highly ordered self-assembly of surfactant molecules. The longer the surfactant chain length, the lower the charge density and the weaker is the hydrophobicity of polyelectrolyte chains are required to form ordered structures. 3) The charge density of the polyelectrolyte is an important parameter determing the structures formed. When the charge density of polyelectrolyte chains decreases the structures of the resulting complexes become less ordered. Both the aggregation number and the radius of the micelles inside the complexes decrease with decreasing charge density of the polymer. 4) The flexibility of polyelectrolyte chains affects the shape of structural elements formed by the self-assembly of polymer-bound surfactant molecules. The surfactant molecules bound by stiff polymer chains prefer to form the structural element of cylinders, while those bound by more flexible polymer chains can form spheres. 5) Temperature has also a clear effect on the stability of the ordered structures. At higher temperatures ordered structures with lower stability are formed, and above certain temperatures, the formation of highly ordered structures inside the complexes can be totally prevented. 


\section{5. Basics of the research methods used in this study}

\section{5. 1. Surface tension}

There is a simple relationship between a change in the surface tension and the surface excess of surface-active agent. For a binary system the relation can be written:

$$
d\left(=-{ }^{\prime}{ }^{(1)} d: 2\right.
$$

where, ' ${ }^{(1)}$ is the excess surface concentration of component 2 , and $:_{2}$ is the chemical potential of component 2 . This is so called Gibbs adsorption equation.

It was (Jones, 1967), who studied the properties of mixed poly(ethylene oxide) (PEO)/sodium dodecyl sulphate (SDS) systems and first formalised the concept, in a system of fixed polymer concentration and increasing amounts of surfactant, using two critical concentrations, viz. $T_{1}$ and $T_{2}$, of the surfactant (Figure 6). When an increasing amount of surfactant is added, the concentration of surfactant on the water/air interface increases. This is seen as a reduction in surface tension. $T_{1}$ represent the concentration at which the cooperative interaction between surfactant and polymer first occurs. The surface concentration of the surfactant does not increase, because all added surfactant associates with the polymer. This results in a flat part in the surface tension curve. $T_{2}^{\prime}$ is the concentration at which the polymer becomes saturated by the surfactant and the surface tension starts to decrease again. At $T_{2}$, the surfactant concentration reaches the $\mathrm{cmc}$; and all added surfactant forms micelles. The concentration of surfactant monomers at the surface does not increase any more and the surface tension remains nearly constant. Jones illustrated the simplicity of the surface tension method when applied to a mixture of a highly surface-active species, the surfactant, and a weakly surface-active species, the polymer. If one makes the reasonable assumption that the surface tension is a sensor of the free surfactant in solution, a method to monitor the concentration changes of noncomplexified surfactant in the mixed systems is thus provided. 


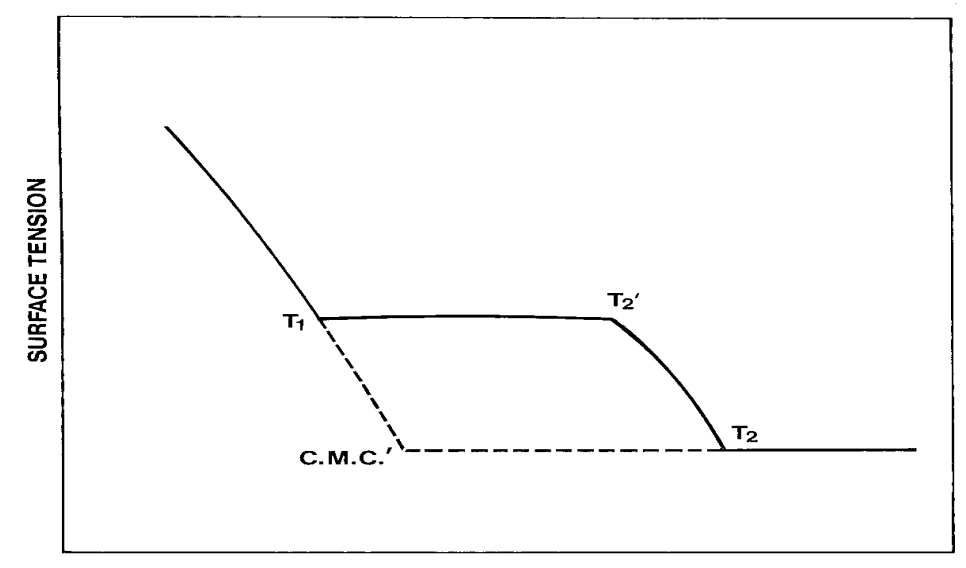

LOG CONCENTRATION

Figure 4. Diagrammatic surface tension/concentration plot of a surfactant in the presence of a complexing polymer. From (Goddard and Anabthapadmanadhan, 1993).

A system which has been widely studied by the surface tension method is poly (vinyl pyrrolidone) (PVP)/SDS as illustrated in the works of (Lange, 1971) and (Murata et al., 1973). Another system which is much studied by surface tension method is the system of Polymer JR (cationic hydroxyethyl cellulose derivate)/SDS, studied by (Goddard et al., 1976). They detected: 1) A synergistic lowering of the surface tension at very low surfactant concentration, implying the formation of a highly surface active complex; 2) The persistence of a low surface tension even in the zone of high precipitation where most of the originally added SDS is out of the solution; 3) Eventual coincidence with the surface tension curve of the polymer-free surfactant system in the micellar region. The observed phenomena can be explained in terms of the diagrams shown in Figure 5, representing progressive uptake of surfactant by the polymer. 


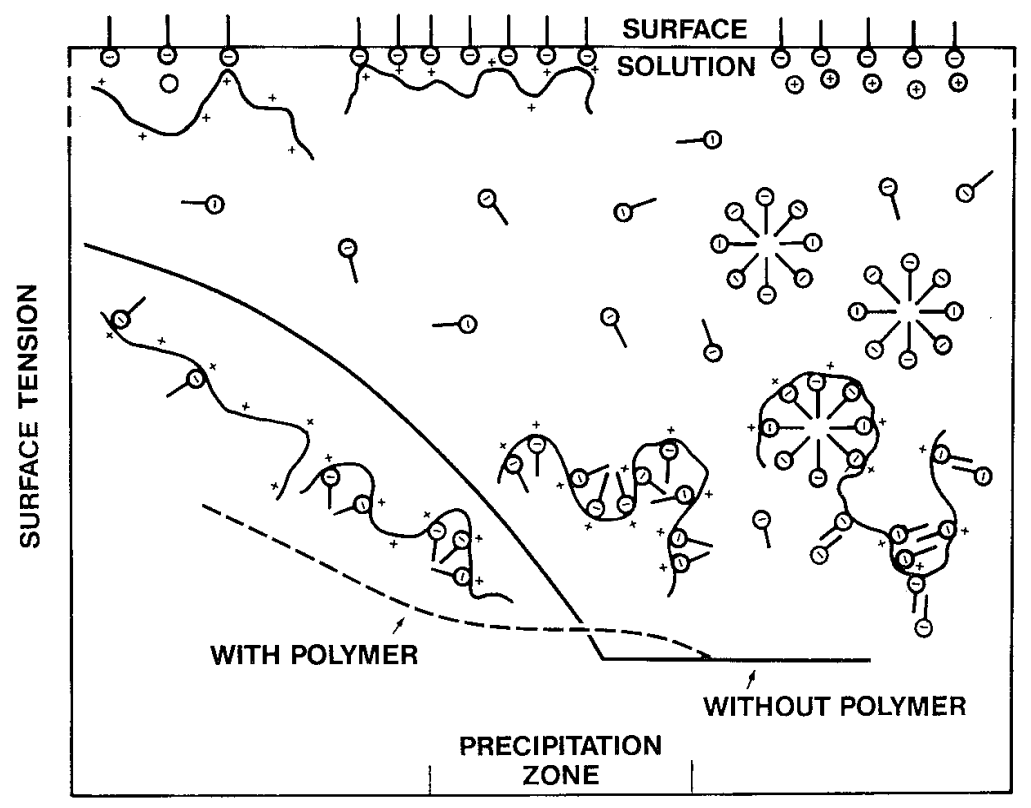

CONCENTRATION SURFACTANT

Figure 5. Conditions in bulk and surface of a solution containing a polycation (fixed concentration) and anionic surfactant. Full line is the hypothetical surface tension/concentration curve of the surfactant alone; dotted line is that of the mixture with polycation. Simple countercations are depicted only in surface zone. From (Goddard and Anabthapadmanadhan, 1993).

\section{5. 2. Solution viscosity and rheology}

One of the most significant aspects of polymer-surfactant systems from a practical point of view is that of rheology control and viscosity enhancement. The studies can be grouped into three categories: 1) Cross-linking of hydrophobe-modified polymers by surfactant; 2) Polyelectrolytes interacting with ionic surfactants; 3) Using the temperature-dependent solvation of non-ionic polymers to obtain a thermal gelation with ionic surfactants. The following representation will mainly concentrate on the second case i.e. polyelectrolyte-ionic surfactant systems.

Dilute solution viscosity is usually measured in capillary viscosimeters of the Ostwald-Fenske or Ubbelohde type. The measuring of reduced viscosity or viscosity number is widely used as a tool for analysis in polymer/surfactant systems.

The reduced viscosity values are calculated from the efflux times using: 


$$
0_{\text {red }}=\left(\left(t-t_{o}\right) / t\right) / C
$$

where $t$ is the measured efflux time, $t_{o}$ is the efflux time of the pure solvent (water) and $c$ is the weight concentration of the polymer.

The interplay of the polymer charging, related conformational changes, and viscosity increases is well recognised. In systems of uncharged polymer and ionic surfactant, many studies have been carried out using viscosity measurements. Jones reported a steady increase in the relative viscosity of PEO solution on adding increasing amounts of SDS (Jones, 1967). A clear change in viscosity occurred at a concentration $T_{2}$, but no slope change was present near $T_{1}$. In the same way, (Lange, 1971) observed an increase in viscosity of PVP on addition of SDS. In this case, the effect was recorded as an increase in specific viscosity that occurred in the vicinity of $\mathrm{T}_{1}$ for this system. These results clearly imply a change in polymer conformation, viz. an expansion of the polymer coils, on association with the charged surfactant, as reported also by Nagaran and Kalpakci of PEO/SDS systems (Nagaran et al., 1982), and by (Tadros, 1974) for PVAc/DDBS systems, respectively. A systematic investigation of the variation of the viscosity of PEO solutions, covering a range of molecular weight and concentration, as a function of added SDS concentration, has been described by (Francois et al., 1985). Their plots of reduced viscosity show a sudden increase at a concentration $\left(\mathrm{T}_{1}\right)$ of SDS, which was independent of the polymer concentration, and a levelling off at a concentration $\left(\mathrm{T}_{2}\right)$, which increased with polymer concentration (Figure 6). 


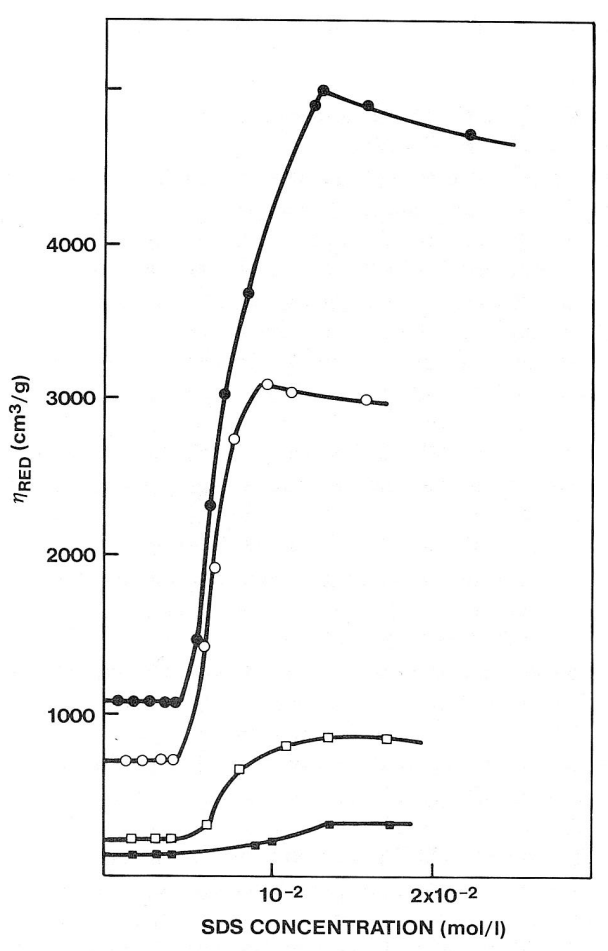

Figure 6. Reduced viscosity of poly(ethylene oxide) as a function of SDS concentration: (!)

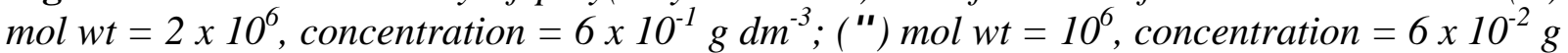
$\mathrm{dm}^{-3}$; (Q) $\mathrm{mol} w \mathrm{wt}=2 \times 10^{5}$, concentration $=5 \times 10^{-1} \mathrm{~g} \mathrm{dm^{-3 }}$; (\#) $\mathrm{mol} w t=7 \times 10^{4}$, concentration $=5 \times 10^{-1} \mathrm{~g} \mathrm{dm}^{-3}$. (Fancois et al., 1985)

Several references may be found in the literature to the compaction of polyelectrolytes on binding of oppositely charged surfactants (Musabekov et al., 1983). Viscosity measurements have been carried out by (Abuin and Scaiano, 1984) on mixtures of PSS of $\mathrm{M}_{\mathrm{w}}$ of 130000 and DTAB. When added to solution of $0.36 \mathrm{w} \%$ PSS, DTAB in the pre-precipitation zone brought about a progressive reduction in the viscosity of the solutions. For example, $5.8 \mathrm{mM}$ DTAB was found to drop the reduced viscosity of PSS by a factor of ten, far exceeding the reduction effected by addition of the simple analog "surfactant", tetradecyltrimethylammonium bromide. The explanation offered was that coiling of the flexible "vinyl" backboned polyelectrolyte occurs around small clusters of the surfactant, which form under these conditions. Similar effects have been obtained by (Bekturov et al., 1984), who studied the viscosity characteristics of a series of amphoteric polymers on addition of anionic (SDS) or cationic (CTAB) surfactants to their solutions.

(Goddard et al., 1982) and (Leung and Goddard, 1985) have reported viscosimetric and rheological studies on two cationic polymers, viz. a cationic cellulosic (Polymer JR) and an acrylamide/\$-methacryloxyethyltrimethylammonium chloride copolymer (Reten, Hercules) 
within a range of polymer and added SDS concentrations. Considerable differences in behaviour between the two polymers were found. At the $1 \%$ Reten level, no change was detectable in viscosity at all levels of added SDS but, by contrast, the viscosity of $1 \%$ Polymer JR solutions increased over 200-fold (Figure 7).

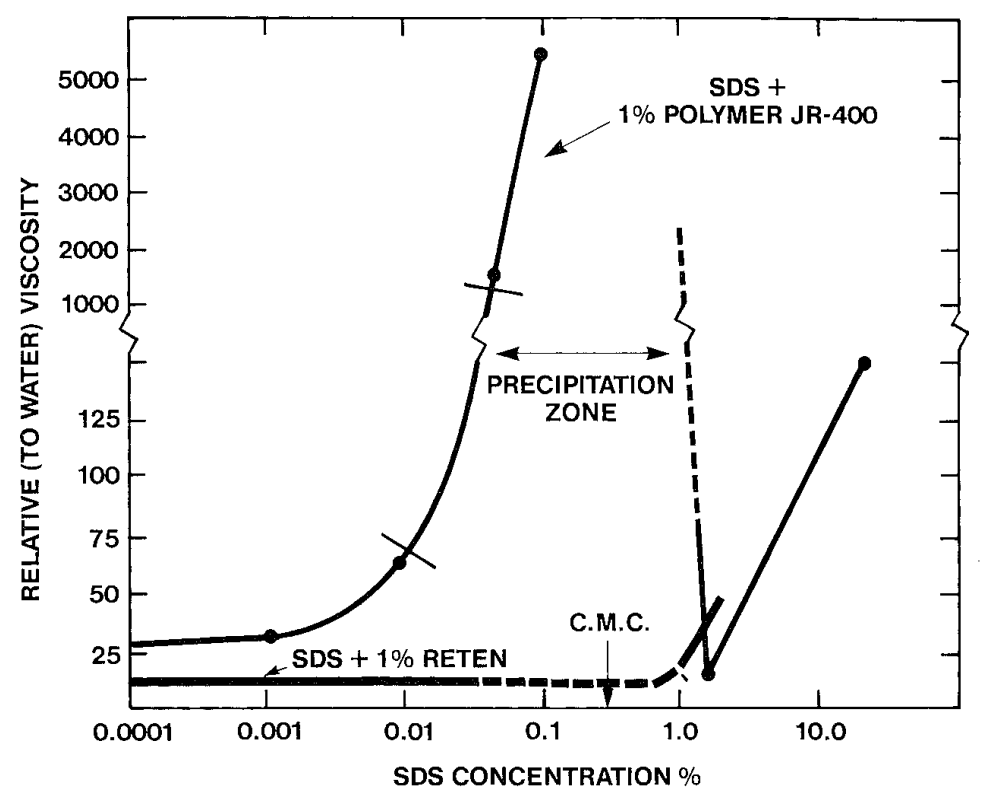

Figure 7. Relative viscosity of $1 \%$ Polymer JR400 and 1\% Reten 220 as a function of SDS concentration. From (Goddard and Leung, 1982).

Under the same conditions, the viscosity of $0.1 \%$ Polymer JR solutions decreased somewhat. These results suggested little change in conformation accompanied the binding of SDS to Reten but that intermolecular association was promoted in the solutions of the cationic cellulose at higher concentration and intramolecular association at the lower concentrations. The results of viscosity measurements were interpreted so that the low-viscosity form is a somewhat tangled necklace structure of SDS micelles and polymer chains, and the higherviscosity form is a more open structure with double layer of adsorbed SDS molecules.

A careful rheological characterisation of the JR-400/SDS gels showed that the elastic properties dominate over the viscous ones even down to very low frequencies in oscillatory measurements. The strength of these gels increases with increasing charge density of the polymer. The structure of the complexes is a stiff network i.e. the polymer molecules are linked together via surfactant aggregates forming a network structure. 
(Thalberg, 1990b) has studied the rheological properties of hyalouran/polyacrylate- $\mathrm{C}_{\mathrm{n}} \mathrm{TAB}$ complexes (Figure 8). The complexes were also found to be transparent gels. The rheological characterisation of these gels shows that the loss modulus dominates at low frequencies and the elastic modulus at high, but indicates no fundamental difference to concentrated solutions of the polymer alone. From the time-resolved fluorescence quenching and NMR self-diffusion studies, a clear picture of the structure of these complexes arises. It is an entangled polyelectrolyte solution with the surfactant micelles placed in the meshes of the network.

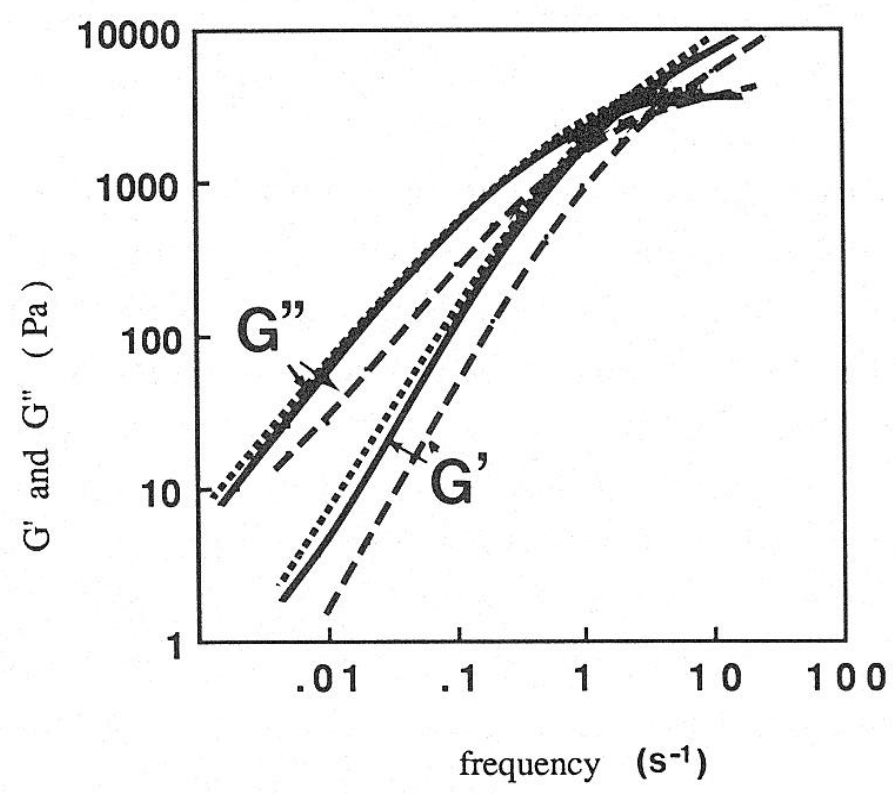

Figure 8. Storage $\left(G^{\prime}\right)$ and loss $\left(G^{\prime}\right.$ ') moduli as a function of oscillation frequency. Dashed lines NaHy (12w\%), full lines NaHy $(12 w \%)+C_{14} T A B(25 w \%)$, dotted lines concentrated complex phase of $\mathrm{NaHy}$ and $\mathrm{C}_{14} \mathrm{TAB}$.

Thus, what is the reason for these differences and hence the rheological properties of these two polylectrolyte-surfactant complexes? The most obvious reason is the stiffness of the polymer chain. Cellulose chains are very rigid which makes them very hard to wrap around the associating surfactant aggregates. Otherwise, the associated micelles can easily act as links between different polymer chains. Hyalouronic and polyacrylate chains are more flexible and are able to wrap around the micelles. In this case, the formation of intermolecular bridges via surfactant micelles between separate polyelectrolyte molecules is very difficult. Another important parameter favouring network formation is, of course the charge density of the 
polyelectrolyte.

\section{5. 3. Dynamic light scattering (DLS)}

Dynamic light scattering has become an important tool in the study of polymer solutions over the last decades. The rationale for this is that it provides data on the molecular mobility and diffusion coeffients of macromolecules, which are of increasing conceptual and practical importance. This data can also be interpreted directly in terms of the size and configuration of the polymer molecule.

It is possible to record the scattering intensity from tiny scattering volumes in time intervals as short as $50 \mathrm{~ns}$. This enables the following of the motion of macromolecules in solution. This is achieved by the construction of a time correlation function (Pecora, 1975).

$$
G_{2}(t)=\langle i(0) i(t)\rangle
$$

where the scattering intensity at time zero is compared with that at a delayed time $t \cdot 10^{-6} \mathrm{~s}-$ $10^{-3} \mathrm{~s}$. This intensity correlation function is difficult to interpret, but can, under most conditions be converted into a correlation function $g_{1}(t)$ of the scattered electric field so that

$$
G_{2}(t)=A+\left(B g_{1}(t)\right)^{2}
$$

with

$$
g_{1}(t)=S(q, t) / S(q)
$$

where $S(q, t)$ is the dynamic structure factor, which is given by

$$
S(q, t)=33<\exp \left(i q^{*} r_{j}(0)-r_{k}(t)^{*}\right)>
$$

Here, $q$ is the magnitude of scattering vector

$$
q=(4 \mathrm{~B} / 8) \sin 2 / 2
$$


and $r_{j}(0)$ is the position of the $j^{t h}$ scattering element at time zero, while $r_{k}(t)$ is that of the $k^{\text {th }}$ element at time t. $S(q)$ is time independent and is the static structure factor

$$
S(q)=<33 \exp \left(i q^{*} r_{j}(0)-r_{k}(0)^{*}>\right.
$$

In most cases, there is a very limited knowledge of the required space-time distribution functions, and only the simplest cases are known. One example is that of small particles with a narrow weight distribution, and for mono-dispersal, large, hard sphere. Then we can write

$$
g_{1}(t)=B \exp \left(-^{\prime} t\right)
$$

with $B$ a constant close to unity and a decay constant ' , that is related to the translational diffusion coefficient $D_{T}$ by the equation

$$
'=D_{T} q^{2}
$$

In most cases, simple single exponential behaviour is not observed, and deviations from the straight line of $\log g_{1}(t)$ against $\mathrm{t}$ are apparent. Once $D_{T}$ has been determined, the hydrodynamic radius, $\mathrm{R}_{\mathrm{H}}$, can be calculated from $D_{T}$ using the Stokes-Einstein equation (Einstein, 1956):

$$
D_{T}=k T / 6 \mathrm{BO} R_{H}
$$

where $k$ is Boltzmann's constant, $T$ is the absolute temperature and 0 is the viscosity.

A polydispersed system of spherically symmetric clusters will have a first-order correlation function with contributions from all sizes (Schmitz, 1990).

$$
g_{1}(t)=\mathrm{I}_{o}^{4}\left(F\left(R_{e f f}\right) \exp \left[-\left(q^{2} D_{e f f} t\right)\right] d R_{e f f}\right.
$$

where the weighting factor $F(R)$ is the intensity of light scattered by clusters of size $R$. The effective diffusion coefficient in this equation equals the translational diffusion coefficient for 
$q R<1$. When $q R>>1$ the rotational diffusion coefficient contributes to the decay of correlation function. The intensity distribution function can be related to the number distribution function, $N(R)$, via

$$
F(R)=B^{\prime \prime 2}(R) S(q R) N(R)
$$

where $B$ is a normalization factor and " $(R)$ and $S(q R)$ are the polarisability and form factor for clusters of size $R$ measured at a wave vector $q$.

Substitution of Eq. 24 into Equations 20 and 21, and assuming that $D_{\text {eff }}=k T / 6 \mathrm{~B} 0 R_{\text {eff }}$ yield a Laplace transform relationship between the measured second-order correlation function and the intensity distribution

$$
\left(g_{2}(t)-1\right)^{1 / 2}=\mathrm{I}_{o}^{4} F\left(R_{e f f}\right) \exp \left[-\left(q^{2} k T / 6 \mathrm{~B} 0\right) R_{e f f}^{-1} t\right] d R_{e f f}+*
$$

where $*$ is a constant background contribution to $g^{(1)}(t)$ due to scattering from large impurities or dust.

There are many procedures developed to obtain the intensity distribution of clusters from the measured time correlation function. One of the most frequently used is Contin (Provencer, 1982 and Ju, 1992), which is developed by Provencer. The program uses a constrained Laplace transform to find the smoothest non-negative intensity distribution consistent with the data. A grid of line-widths is laid out with equal spacing in $\log \left({ }^{\prime}\right)$ and a preliminary non-smoothed solution is sought. Thereafter, a penalizing function (regulariser) is added to the model and additional solutions are sought with increasing weight of the regulariser. For each solution, a statistic, the 'probability to rejection', is calculated via comparison of the sum of squared residuals for this degree of regularisation and that obtained in the preliminary calculation. The regulariser used is based on the sum of the second derivates of $G\left({ }^{\prime}\right)$ with respect to ' . The solution for which the 'probability to rejection' is closest to 0.5 is recommended as the 'chosen solution'. All fitting is done to the $\mathrm{g}_{1}$ values, which are calculated from the $g_{2}-1$ values obtained from a homodyne experiment.

Polyelectrolyte systems have specific properties that make the interpretation of the light scattering data difficult (Nicolai, 1989). The polyelectrolytes have small counterions surrounding each polyion, which screens the surface charge of the polyion. This long-range effect is to reduce the apparent charge on the polyion and thereby reduce the magnitude of the 
direct polyion-polyion interactions. The role of the small ions can be classified as passive or active, depending on whether they affect the equilibrium or dynamic properties of the polyion. As passive components, the small ions are assumed to instantaneously relax to their equilibrium distribution, and therefore they act to screen the interactions between the polyions. The second effect is an indirect coupling of the polyions. That is, the dynamics of the polyions become statistically coupled by way of overlapping ion clouds. As active components, the small-ion dynamics can have a significant influence on the dynamics of the polyions.

Especially at very low ionic-strength solutions, polyions are found to diffuse at the same rate as their counterions. Decreasing the ionic strength decrease the observable diffusion rate of the polyions by increasing the repulsion between the charged segments. It has also another effect by expanding the counterion layer and thus increasing the apparent friction factor and so decrease the diffusion rate. The intensity of light-scattering by a charged particle is also reduced relative to the neutral particle. This makes ligh-scattering experiments of polyelectrolytes in dilute solutions even more difficult. When the added electrolyte concentration is lower than the equivalent polyelectrolyte concentration, the intensity correlation functions of polyelectrolyte solutions are no longer of the unimodal type but display a bimodal behaviour, with two characteristic decay rates termed fast and slow. Both a slow and a fast decay rate are found for rod-like and flexible polyelectrolyte molecules such as DNA (Wang et al., 1991) and NaPSS (Sedlak et al., 1992). It has been demonstrated for quaternised poly(2-vinylpyridine) (Förster et al., 1990) that for polyelectrolyte solutions without added salt the intensity correlation functions are unimodal at very low polyelectrolyte concentrations and bimodal at the higher ones.

For polyelectrolyte solutions containing an abundant amount of salt, the apparent cooperative diffusion coefficients $\left(\mathrm{D}_{\mathrm{app}}\right)$ determined from the correlation functions are discussed in analogy to those observed in solutions of uncharged macromolecules. In these systems, two polyelectrolyte concentration regitimes can be separated. In the dilute regitime, in which the polyelectrolyte coils are well separated and behave like independent entities, the cooperative diffusion coefficient depends on the size of the individual coils. In the semi-dilute regitime, the polyelectrolyte chains form a transient physical network and the cooperative diffusion coefficient depends on the size of this network. Experimental evidence for this behaviour has been given by (Koene et al., 1983 a, b, c) for solutions of poly(styrenesulphonate) (NaPSS) and (Smits et al., 1993) for solutions of linear poly(ethyleneimine) chloride (LPEI). Due to 
these difficulties, light-scattering techniques have seldom been employed to elucidate changes in systems of polymers and surfactants. Dubin and co-workers (Dubin et al. 1988, 1990) have described light-scattering measurements on a negatively charged surfactant in solution with a neutral polymer, and on the complex formed between mixed micelles and an oppositely charged polyelectrolyte. There is also an article by (Fundin et al., 1994) where polymer/surfactant interactions in a system of sodium poly(styrenesulfonate) and CTAB are studied by light-scattering measurements.

\section{5. 4. Small Angle X-ray Scattering (SAXS) and Small Angle Neutron Scattering (SANS)}

Small angle scattering arising from inhomogeneities in the refractive index within a material coherently scatter radiation in a forward direction. The intensity distribution as a function of angle is dependent on the shape, the size, the concentration, and the smoothness of the inhomogeneities. Small angle scattering data can be analysed directly by curve fitting and by comparison to theoretical models. Small angle scattering is used to elucidate microstructural information in amorphous non-heterogeneous materials on length scales ranging from a few ångströms to a few micrometers.

2. 5. 4. 1. Basics of the theory of small angle scattering

We assume a plane monochromatic wave $A_{o} \exp \left(i \boldsymbol{k}_{o} \boldsymbol{r}\right)$ incident at the point scattering centre 0 , which generates a secondary spherical wave. Then, at some observation point, the resulting wave is given by

$$
A_{o} \exp \left(i \mathbf{k}_{\mathrm{o}} \mathbf{r}\right)+\left(A_{o} b / r\right) \exp (i \mathbf{k r})
$$

where $\boldsymbol{k}_{\boldsymbol{o}}$ and $\boldsymbol{k}$ are the incident and scattering wave vectors with $\boldsymbol{*}_{\boldsymbol{o}}{ }^{*}=\boldsymbol{k}^{*}=2 \mathrm{~B} / 8,8$ denoting the wavelength, $A_{o}$ and $\left(A_{o} b / r\right)$ are the scattering amplitudes of the two waves, and $r$ is the vector which determines an observation point $L$ corresponding to the scattering centre 0 . The stronger is the interaction between the incident wave and the point centre 0 , the greater the constant $b$. This quantity has the dimension of length and is called the "scattering length", or "the scattering amplitude", of the centre.

We consider the scattering of a plane monochromatic wave of X-rays or neutrons by a real 
object consisting of an accumulation of nuclei and electrons - a group of point scatterers. The scattering ability of the object can be characterised by the scattering density function $\mathrm{n}(\boldsymbol{r})$. In the case of X-ray scattering, $n(\boldsymbol{r})$ represents the distribution of the electric charge density, while in neutron scattering this function represent the nuclear and spin density distribution. The wave interacts with all the nuclei and electrons, which become the sources of secondary waves. The superposition of these waves gives the first approximation to the scattering waves. The use of the first approximation is appropriate for weakly scattering centres or fields only (Landau and Lifshitz, 1985). The wave $\mathrm{R}(\boldsymbol{r})$ scattered by field $\mathrm{n}(\boldsymbol{r})$ is sought as a solution of the wave equation.

$$
\left.[)+k_{0}^{2}+\varangle(\mathbf{r})\right] \mathrm{R}(\mathbf{r})=0
$$

where $k_{0}=*_{k_{0}} *$ represents the wave number for the incident wave in free space, ) is the Laplace operator, and < is the parameter specifying the strength of the interaction with a potential field. The solution of this equation is sought as power series in $<$ To the first approximation and $*_{\boldsymbol{r}}-\boldsymbol{r}{ }^{*}$ is very large compared the scattering area i.e. the case of Fraunhofer diffraction the scattering vector is in the form

$$
\psi^{(0)}(r)+\psi^{(1)}(r)=A_{0} \exp \left(i k_{0} r\right)+\frac{A_{0} \vee}{4 \pi} \frac{\exp \left(i k_{0} r\right)}{r} \int \varphi\left(r^{\prime}\right) \exp (i s r) d r^{\prime}
$$

where $s=k-k_{0}$ represents the scattering vector and $*_{s} *=(4 \mathrm{~B} \sin 2) / 8,22$ being the scattering angle.

Comparison of Equations 26 and 28 shows that, for the potential field $\mathrm{n}(r)$, the function

$$
f(s)=\frac{v}{4 \pi} \int \varphi(r) \exp (i s r) d r
$$

plays the same role as that of factor $b$ in the case of the point centre. Thus, $f(s)$ is the amplitude of elastic scattering by the field $n(\boldsymbol{r})$. This expression is a first Born approximation, in other words, the single-scattering approximation where it is assumed that there is no interaction between separate particles. This expression represents a Fourier transform. Solution of the 
inverse problem - calculation of $\mathrm{n}(\boldsymbol{r})$ using the known function $f(s)$ - is given by inverse Fourier transform

$$
\varphi(r)=\frac{1}{2 \pi^{2} v} \int f(r) \exp (-i s r) d s
$$

In an experiment we cannot measure the scattering amplitude, but only the flow of scattering energy or the number of scattered particles, proportional to the square of scattering amplitude:

$$
\frac{d W}{d \Omega}=\frac{A_{o}^{2}}{r^{2}}|f(s)|^{2}=\frac{A_{0}^{2}}{r^{2}} I(s)
$$

where $S$ represents a solid angle. The function $I(s)$ is scattering intensity (term used in X-ray scattering) or scattering cross section (for neutron scattering).

One significant feature of an object is the total scattering intensity (or total scattering cross section), which is obtained by integrating Equation (31) over all the angles:

$$
\sigma=\int_{\Omega} \frac{I(s)}{r^{2}} d \Omega
$$

The main problem in the structure analysis of matter is the reconstruction of the scattering density distribution according to the measured function $I(s)$.

\section{5. 4. 2. Scattering by simple objects}

The relationships for Fourier integrals are very useful when calculating scattering amplitudes involving objects of simple shape:

Rectangular parallelepiped. For homogenous palallelepiped (scattering density $\mathrm{n}_{0}$ ) with edges $a, b$ and $c$ we can write:

$$
\varphi(r)=\varphi(x, y, z)=\varphi_{0} \Pi(x, a) \Pi(y, b) \Pi(z, c)
$$


where function $A$ is a Fourier image. The scattering amplitude is the given by

$$
f(q)=\varphi_{0} V \delta(a, X) \delta(b, X) \delta(c, Z)
$$

where $V=a b c$ represent the parallelepiped volume and $*(u, v)=\sin (\mathrm{B} u v) / \mathrm{B} u v$. This general relationship relates the linear characteristics of real and reciprocal spaces and represents the general definition of the resolution limits of any diffraction experiment. If $a, b, c 64$ we obtain:

$$
f_{\infty}(q)=\varphi \delta(X) \delta(Y) \delta(Z)
$$

i.e., the whole scattering is concentrated at point $q=0$. Thus, the scattering intensity of a parallelepiped is given by the relationship

$$
I(q)=\varphi_{0}^{2} V^{2} \delta^{2}(a, X) \delta^{2}(b, Y) \delta^{2}(c, Z)
$$

Homogenous thin plate. This case is obtained from previous one when $c 60$. Thus, if $S=a b$ (the area of plate) and $a, b 64$ (infinitely thin plate) one obtains

$$
f(q) \rightarrow \varphi_{o} \delta(X) \delta(Y)
$$

i.e. scattering amplitude differs from zero only along the straight line $X=0, Y=0$.

Homogenous thin rod. This object is also a particular case of homogenous parallelepiped when a, $b 60$, so

$$
\begin{aligned}
& \varphi(r)=\varphi_{0} \delta(x) \delta(y) \Pi(z, c) \\
& f(q)=\varphi_{0} c \delta(c, Z)
\end{aligned}
$$

if $c 64$ (infinitely long rod)

$$
f(q)=\varphi_{0} \delta(Z)
$$


i.e. the whole scattering is concentrated in the plane $Z=0$.

Spherically symmetric body. When $\mathrm{n}(\boldsymbol{r})=\mathrm{n}(r)\left(r={ }^{*} *\right)$, then using spherical coordinates one may write

$$
f(s)=4 \pi \int_{0}^{\infty} \varphi(r) \frac{\sin (s r)}{s r} r^{2} d r
$$

where we recall that $s=2 \mathrm{~B} q=4 \mathrm{~B} \sin 2 / 8$. It is clear that for spherically symmetric bodies, the scattering patterns also have spherical symmetry. For scattering by a homogeneous solid sphere

$$
\varphi(r)=\Pi(r-R)=1, r \leq R ; 0, r>R
$$

and one may obtain the following important relationship by direct calculation:

$$
f(s)=3(s R)^{-3}[\sin (s R)-s R \cos (s R)]=\theta(s R)
$$

where $f(0)=1$ (normalised scattering amplitude).

There are several good textbooks published on the theory of SAXS and SANS where the theory is discussed in more detail (Glatter and Kratky, 1992, Brumberger, 1995, Feigin and Svergun, 1987).

\section{5. 4. 3. Structure analyses of ionic micelles with SANS}

Charged micelles can be modelled as a population of particles that interact through a screened Coulomb potential. For a population of slightly anisotropic or polydispersed particles of number density $n$, the differential cross sections of neutron scattering per unit volume, $d \mathrm{G}(q) / d \mathrm{~S}$, can be written as (Feigin and Svergun, 1987)

$$
\frac{d \Sigma(q)}{d \Omega}=n\left\{<|P(q)|>^{2} S(q)+\left[<|P(q)|>^{2}-<|P(q)|>^{2}\right]\right\}
$$


where the scattering vector $q$ is defined by

$$
q=\frac{4 \pi \sin \theta}{\lambda}
$$

and 22 is the scattering angle and 8 is the wavelength. The form factor $P^{2}(q)$, which expresses the scattering cross-section of a single spherical particle of radius $r$, is

$$
P(q)=\int\left(\rho(r)-\rho_{s}\right) \exp (i q r) d v
$$

$\mathrm{D}(r)$ is the scattering length density of the particle and $\mathrm{D}$ is the scattering length density of the solvent. The structure factor $S(q)$ is given by

$$
S(q)=1+\frac{1}{V} \int[g(r)-1] \exp (i q r) d v
$$

where $g(r)$ is the interparticle correlation function and $V$ is the volume of solution per particle. The micelles are assumed to be monodisperse, spherical or prolate, core-and-shell ellipsoids of volume $V_{2}$ with semiaxes $a, b, b(a / b=()$. The volume of the core, which consists of hydrocarbon chains, is $V_{l}$ and its scattering length density is $D_{l}$. The volume of the shell, which contains the polar head groups, is $V_{2}-V_{l}$ and its scattering length density is $\mathrm{D}_{2}$. Then the single particle scattering function is given by:

$$
P(q)=\int_{0}^{\pi / 2}\left[V_{1}\left(\rho_{1}-\rho_{s}\right) F\left(q, R_{1}\right)+V_{2}\left(\rho_{2}-\rho_{s}\right) F\left(q, R_{2}\right)\right] \sin \beta d \beta
$$

where

$$
F(q, R)=3 \frac{\sin X-X \cos X}{X^{3}}
$$

with

$$
X=q R \sqrt{\sin ^{2} \beta+\gamma^{2} \cos ^{2} \beta}
$$


Let the mean aggregation number of micelles to be $N_{a}$. The mean volume of the core, $V_{l}$, can then be calculated from molecular group volumes. For sodium alkanoate micelles for example:

$$
V_{1}=N_{a}\left[v\left(\mathrm{CH}_{3}\right)+(<n>-1) v\left(\mathrm{CH}_{2}\right]\right.
$$

while the volume of the shell, $V_{2}$, is given by

$$
V_{2}-V_{1}=N_{a}\left\{v\left(C O O^{-}\right)+\Phi_{H G} v\left(D_{2} O\right)+(1-\alpha)\left[v\left(N a^{+}\right)+\Phi_{N a} v\left(D_{2} O\right]\right\}\right.
$$

where

$-\langle n\rangle$ is the average number of carbon atoms in the hydrocarbon chains of the micelle

- $\left.\left.\left.4 \mathrm{CH}_{2}\right), \varangle \mathrm{CH}_{3}\right), 4 \mathrm{COO}^{-}\right)$are the volumes of, respectively, methylene, methyl and head groups in the surfactant

- $\left.4 \mathrm{Na}^{+}\right)$and $\left.4 \mathrm{D}_{2} \mathrm{O}\right)$ are the volumes of sodium ions and solvent molecules bound to the surfactant

- $\mathbf{j}_{H G}$ and $\mathbf{j} N a$ are the hydration numbers of the head groups and of the sodium ion, respectively

- " is the degree of dissociation of the surfactant molecules in micelle

The second term of Equation (44) can be calculated using the decoupling approximation of (Kotlarchyk and Chen, 1983), i.e. it is assumed that there is no correlation between interparticle separation and particle size, and no correlation between inter-particle distances and particle orientation. This assumption is reasonable for dilute solutions of charged micelles if ( is not much bigger than one. The rescaled mean spherical approximation (RMSA) by (Hansen and Hayter, 1982) can be used to calculate the structure factor for dilute dispersions of charged colloidal particles:

$$
S(q)=\frac{1}{1-24 \eta G\left(q, R_{H S}, \eta_{H S}, Z_{e f f}\right)}
$$


where 0 is volume fraction of particles. The exact expression of $G\left(q, R_{H S}, 0_{H S}, Z_{e f f}\right)$ the function of effective hard sphere radius, hard sphere volume fraction and effective charge can be found in (Hayter and Penfold, 1981) and (Hansen and Hayter, 1982).

If the interparticle interactions are assumed to be due to interactions between the diffuse double layer surrounding the micelles, then the interaction potential at distance $r$ from the centre of a spherical micelle of mean diameter $\langle d\rangle$ is given by

$$
V(r)=\frac{\pi \varepsilon \varepsilon_{0} d^{2} \psi_{0}^{2} \exp [-\kappa(r-<d>)]}{r}
$$

where $\mathrm{R}_{0}$ is the surface potential, $g_{0}$ is the permeability of vacuum, $g$ is the dielectric constant of the solution between micelles, 6 is the Debye-Hückel inverse screening length, determined by the ionic strength of the solution. The surface potential is related to the charge, $z$, on the micelle, to a good approximation by (Verwey and Overbeek, 1948).

$$
\psi_{0}=\frac{z}{<d>\pi \varepsilon \varepsilon_{0}(2+\kappa<d>)}
$$

The average diameter, aggregation number $\left(N_{a}\right)$, axial ratio $\left(()\right.$, average number of charges $\left(e_{0}\right)$ and degree of dissociation (") of the micelles can be obtained by fitting procedure.

\section{5. 4. 4. Determination of molecular structure of liquid crystals by SAXS}

The method is based directly on analysing the diffraction patterns. Braggs condition says that

$$
n 8=2 d \sin 2
$$

where 8 is the wave length of X-ray source, $d$ is the layer spacing, 2 is the scattering angle and $n$ is an integer. The scattering vector, $q$, can determined as

$$
q=d^{-1}=2 \sin 2 / 8
$$


In the small angle X-ray measurements the scattering intensity is measured as a function of scattering vector, $q$. The structure of periodic continuous well-ordered structure normally has 2 to 3 diffraction peaks. These can be used to determine the structure. When the three diffraction maxima associated with the spacing of structure are $d_{1}, d_{2}$ and $d_{3}$, the ratio between the maxima d1/d2/d3 shows the ordering: $1 / 2$ / 4 lamellar, $1 / \% / \%$ hexagonal, $\% / \%$ / \% cubic etc. (Hahn, 1985).

Thus, the structure of liquid crystalline mesophases can be easily determined from the characteristic spacing ratios of the diffraction peaks. The lattice parameter $a$, the micelle radius $r$, the micellar separation $h$, and the surface area per surfactant ion $A$ can be calculated from the values of spacing $q_{1}$, volume fraction of the surfactant ions $\mathrm{N}_{s}$, the mass per surfactant ion $M$, and its density D. For example, in the case of hexagonal mesophase of surfactant

$$
\begin{aligned}
& a=\left(\frac{2}{\sqrt{3}}\right)\left(\frac{2 \pi}{q_{1}}\right) \\
& R=a\left[\phi_{s} \frac{\sqrt{3}}{2 \pi}\right]^{1 / 2} \\
& A=\frac{2 M}{R \rho} \\
& h=a-2 R
\end{aligned}
$$

Possible difficulties in determining of structure parameters arise from the second and higher order reflections.

\section{EXPERIMENTAL}

\subsection{Materials}

\subsubsection{Cationic starch}


Starch is a polymeric carbohydrate consisting of anhydroglucose units linked together primary through "-D-(164) glucosidic bonds. Starch is a heterogeneous material consisting, at the extremes, of two major types of polymers - amylose and amylopectin.

Amylose is essentially linear polymer in which the anhydroglucose units are predominantly linked through "-D-(164) glucosidic bonds. It may contain about 200 to 2000 anhydroglucose units.

Amylopectin is a branched polymer containing, in addition to anhydroglucose units linked together as in amylose through "-D-(164) glucosidic bonds, periodic branches at the carbon-6 position. Each branch contains about 20 to 30 anhydroglucose units. A schematic diagram is shown in Figure 9. Light-scattering measurements indicate molecular weights in the millions. Depending on botanic source of the starch the amylopectin/amylose ratio can vary from 1 to near 0.3 . Potato starch, which was used in this work, contains about $79 \%$ amylopectin and $21 \%$ amylose.

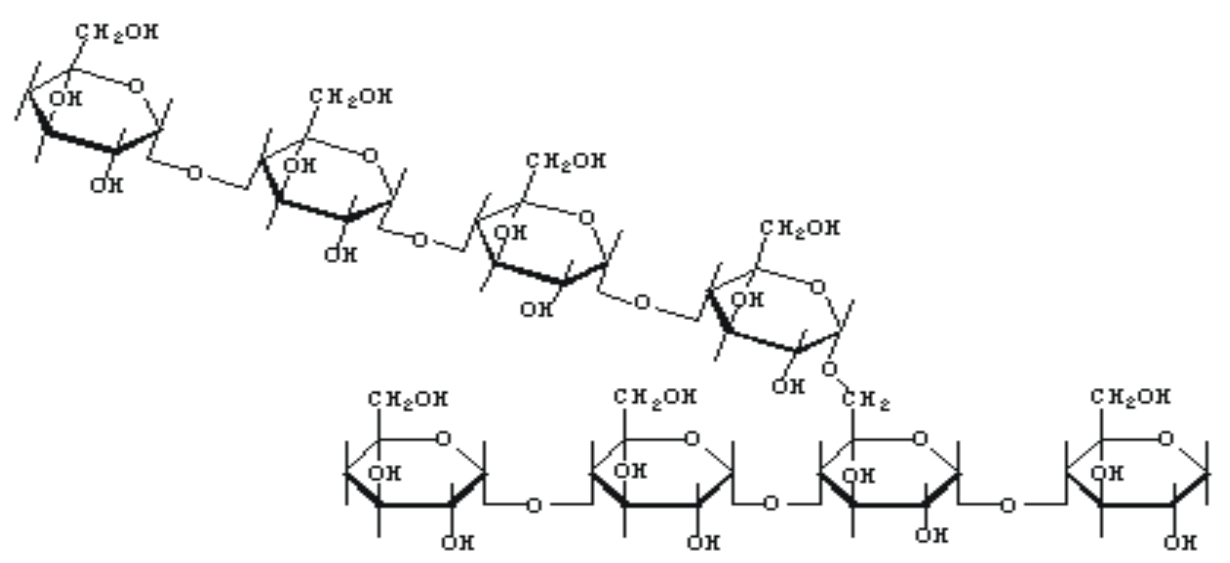

Figure 9. A schematic diagram of the structure of amylopectin, where monoglucose units are linked together via 1,4-" -glycisidic bindings.

The starch, used in these studies, was a quaternary substituated (2-hydroxy-3trimethylammoniumpropyl starch) (Fig.10 and Fig. 11) delivered from Raisio Chemicals, Raisio, Finland. The potato starch was oxidised by sodium hypoclorite before cationisation, thus it was depolymerised to a considerably degree and so it was not possible to separate the amylose and amylopectin fractions in the GPC chromatograms. 


\section{Starch $-\mathrm{O}-\underset{\mathrm{O}}{\mathrm{CH}_{2}} \mathrm{CHCH}_{2} \mathrm{~N}^{+}\left(\mathrm{CH}_{3}\right) \mathrm{Cl}^{-}$}

Figure 10. Quaternary ammonium-group as cationic substituent at cationic starch.

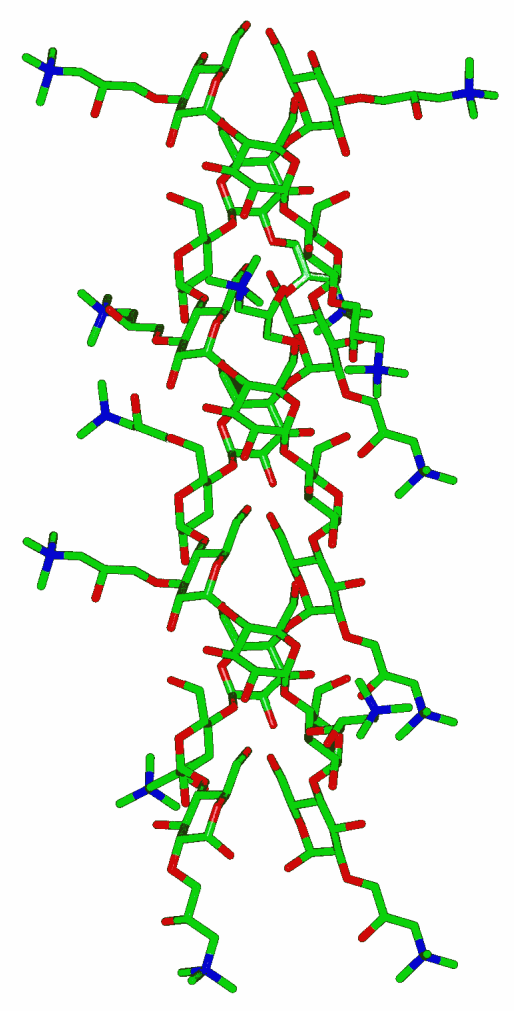

Figure 11. A rod model of helix-like structure of cationic amylose molecule. Green sticks represent the carbon-carbon bindings, the red ones the bindings including oxygen, and the blue ones the quaternary ammonium - carbon bindings in cationic group.

\subsubsection{Surfactants}

Surfactants were sodium dodecyl sulphate (SDS) and different alkanoates with hydrocarbon chain length from $\mathrm{C}_{8}$ to $\mathrm{C}_{22: 1}$. The alkanoates were potassium octanoate, $\mathrm{C}_{7} \mathrm{H}_{15} \mathrm{COOK}$, (KOct) 
and dodecanoate, $\mathrm{C}_{11} \mathrm{H}_{23} \mathrm{COOK}$, (KDod), sodium octanoate, $\mathrm{C}_{7} \mathrm{H}_{15} \mathrm{COONa}$, (NaOct), decanoate, $\mathrm{C}_{9} \mathrm{H}_{19} \mathrm{COONa}(\mathrm{NaDe})$, dodecanoate $\mathrm{C}_{11} \mathrm{H}_{23} \mathrm{COONa}$ (NaDod), palmitate (sodium hexadecanoate), $\quad \mathrm{C}_{15} \mathrm{H}_{31} \mathrm{COONa}, \quad(\mathrm{NaPal}), \quad$ oleate (sodium cis-9-octadecenoate), $\mathrm{C}_{9} \mathrm{H}_{18}=\mathrm{C}_{8} \mathrm{H}_{15} \mathrm{COONa}$, $(\mathrm{NaOl})$ and erucate (sodium cis-13-docosenoate), $\mathrm{C}_{9} \mathrm{H}_{18}=\mathrm{C}_{12} \mathrm{H}_{23} \mathrm{COONa}$, $(\mathrm{NaEr})$.

Sodium dodecyl sulphate (SDS) was analytical grade from Fluka Ag. The surface tension of solutions prepared from the sample as delivered showed a minimum at concentrations just below the $c m c$, indicating that a trace amount of alcoholic impurities was present in the sample. The sample was therefore recrystallised 3 times from ethanol. This procedure removed the impurities causing the minimum. All the alkanoates and alkenoates were synthesized by neutralising the corresponding solution of carboxylic acid (Fluga Ag, purum) in ethanol (20\% solution) with alkyl hydroxide. The soap was recrystallised 3 times from acetone.

The deuterated surfactants were synthesized in the same way as the protonated surfactants from deuterated carboxylic acids $\left(\mathrm{C}_{7} \mathrm{D}_{15} \mathrm{COOH} ; 98 \% \mathrm{D}, \mathrm{C}_{9} \mathrm{D}_{19} \mathrm{COOH} ; 98 \% \mathrm{D}\right.$ and $\mathrm{C}_{15} \mathrm{D}_{31} \mathrm{COOH}$; 98\%D). The deuterated acids were neutralised with $\mathrm{NaOD}$ in $\mathrm{C}_{2} \mathrm{H}_{5} \mathrm{OD}$. All deuterated chemicals were from Medical Isotopes Inc., USA. The purity of all surfactants was verified by measurements of surface tensions. The critical micellisation concentrations $(\mathrm{cmc})$ of the surfactants (SDS: $8.25 \mathrm{mmol} \mathrm{dm}^{-3}$; KOct: $345.0 \mathrm{mmol} \mathrm{dm}^{-3}$; KDod: $16.0 \mathrm{mmol} \mathrm{dm}{ }^{-3}$, NaOct: $398.0 \mathrm{mmol} \mathrm{dm}{ }^{-3}$, NaDe: $110.0 \mathrm{mmol} \mathrm{dm}{ }^{-3}$, NaDod: $27.0 \mathrm{mmol} \mathrm{dm}^{-3}$, NaPal: $1.62 \mathrm{mmol} \mathrm{dm}{ }^{-3}$, NaOl: $0.90 \mathrm{mmol} \mathrm{dm}{ }^{-3}$ and NaEr: $0.07 \mathrm{mmol} \mathrm{dm}^{-3}$ determined from the surface tensions are in good agreement with those reported in literature (Mukerjee and Mysels, 1971).

\subsection{Methods}

\subsubsection{Surface tension measurements}

Surface tension measurements were performed using a ring tensiometer (KSV Instruments Sigma 70). This instrument is computer controlled and makes it possible to make several measurements on the same sample automatically by lowering and rising the vessel containing the solution, thereby passing the ring repeatedly through the weight maximum. The correction factors calculated by (Huh and Mason, 1975) were used to calculate surface tensions. All 
measurements were performed at $25^{\circ} \mathrm{C}$. All solutions were prepared at least $24 \mathrm{~h}$ before measurements. The solubility of starch was ensured by heating the starch solution in an autoclave for $30 \mathrm{~min}$ at $120^{\circ} \mathrm{C}$. In order to avoid the formation of very surface-active acid/soap complexes, the alkanoate solutions were made slightly alkaline before measurements $(\mathrm{pH}=$ 10.5 ) by adding sodium or potassium hydroxide. All the vessels were cleaned using aqua regia, ion-exchanged water and distilled water. The ring and the measuring vessel were treated with a gas flame after measurements. The samples were allowed to stabilize $30 \mathrm{~min}$ in the measuring vessel before measurement. In spite of these precautions, the reproducibility between measurements on the same sample was only of the order $\pm 0.5 \mathrm{mNm}^{-1}$. This uncertainly is at least partly due to the adsorption of starch on the ring and the ring becoming hydrophobic due to adsorption of surfactants. These problems are well known weaknesses of the ring method. The reproducibility between measurements on different samples was about $\pm 1.0 \mathrm{mNm}^{-1}$. This uncertainly is obviously due to the very low concentrations of starch used $(0.001-0.1$ weight $\%)$.

\subsubsection{Viscosity measurements}

Viscosities were determined with an Ubbelohde capillary viscometer system (Schott-Geräte, AVS 350). This instrument is computer controlled and makes it possible to take several measurements on the same sample automatically by repeating filling of the capillary followed by recording of the flow time. The correction factors of Hagenbach were used to calculate the real efflux times. The viscometer was immersed in a bath thermostat to $(298.15 \pm 0.01) \mathrm{K}$. All solutions were prepared at least $24 \mathrm{~h}$ before measurements were made. The solubility of starch was ensured by heating the starch solutions in an autoclave for $30 \mathrm{~min}$ at $120^{\circ} \mathrm{C}$. All starch samples were also filtered through a $3.0: \mathrm{m}$ pore size membrane to remove dust and other macroscopic impurities. The viscosimeter was cleaned using aqua regia, ion-exchanged water and distilled water. The samples were allowed to equilibrate $10 \mathrm{~min}$ in the thermostat set before measuring. The reproducibility between measurements of efflux times on the same sample was normally of the order $\pm 0.01 \mathrm{~s}$. The only difficulties were found at certain surfactant/CS ratios when the aggregates formed very mobile flocs, which tended to stick in the capillary. Another problem, which occurred at some surfactant concentrations, was the formation of bubbles in the samples. This could partly be avoided by draining the capillary fully between measurements. 
The results of the viscosity measurements are presented as reduced viscosity values, calculated from the efflux times (Equation 13)

\subsubsection{Dynamic light scattering}

A Coulter Instruments N4MD light-scattering instrument was used. The light source is a $4 \mathrm{~mW}$ He-Ne laser operating at $632.8 \mathrm{~nm}$. For detection fibre optics are used with the possibility of using 6 different scattering angles $\left(15.2-90^{\circ}\right)$. The correlator-unit has 80 quasi logarithmicallyspaced channels, spanning a time scale of 3072 equivalent linearly-spaced channels. Samples were prepared the same way as for viscosity measurements. To avoid dust, all starch samples were also filtered trough a membrane with $3.0: \mathrm{m}$ pore size. The temperature of the sample chamber was adjusted to $(298.15 \pm 0.2) \mathrm{K}$. All measurements were performed at scattering angle of $90^{\circ}$ in order to minimize the scattering from macroscopical impurities. The particle size distributions were calculated by Contin (Prowencer, 1982 and Ju et al. 1992) analysis program. The reproducibility of the mean particle sizes was of the order $\pm 2 \%$ and the particle size distributions no better than $\pm 10 \%$.

The difficulties observed were due to the very high polydispersity of the samples, which makes analysis of the scattering function very difficult. The starch particles also tend to aggregate, resulting in the formation of particles with high scattering power. This combined with the fact that using He-Ne laser, the lower range of the detection is about $3 \mathrm{~nm}$, led to a situation in which micelles were not detectable.

\subsubsection{Electrophoretic mobility}

Electrophoretic mobility (zeta potential) measurements were performed by Coulter Electronics, DELSA 440, Doppler electrophoretic light-scattering analyser. This instrument makes measurements at four angles simultaneously $\left(7.5^{\circ}, 15^{\circ}, 22.5^{\circ}\right.$ and $\left.30^{\circ}\right)$. The system has four separate detectors and four independent 256-channel analysers. The light source of this instrument is a He-Ne laser operating at $632.8 \mathrm{~nm}$. The measurements were performed at constant ionic strength and the sample preparation was carried out in the same way as in the viscosity and DLS measurements. 


\subsubsection{Phase equilibrium determination}

Weighed amounts of starch solution, surfactant and distilled water were added to tightly closed test tubes. The tubes were equilibrated by continuously turning over at a fixed temperature for 7 days. The tubes were then centrifuged for $30 \mathrm{~min}$ at $1600 \mathrm{~g}$ in order to separate the solution and the complex phases. After centrifugation, the samples were again allowed to equilibrate at the same temperature for 7 days. The gel and the solution phases were separated by careful decantation of the solution. The viscosity of the solution was low, so that separation in this way was relatively easy. The composition of the both phases was analysed by determining the dry content of the complex phase by weighing and the amount of the nitrogen by Kjeldahl analysis (Bradstreet, 1965). The amount of the surfactant was calculated as the difference between the amount of starch and the total amount of dry matter in the samples.

The composition of the supernatant phase was analysed by determining the CS concentration by ordinary spectrophotometric methods used commonly to determine the total carbohydrate content. The amount of surfactant in the supernatant phase was determined by a standard gas chromatographic method of carboxylic acid analysis. CS has $\mathrm{Cl}^{-}$as a counter ion and, the surfactants $\mathrm{Na}^{+}$or $\mathrm{K}^{+}$, thus there is also $\mathrm{NaCl}$ or $\mathrm{KCl}$ forming due to the association. The electrolyte concentration of separate phases was not determined, but total salt content is, of course, directly dependent on the amount of associated surfactant.

\subsubsection{Rheology}

A Bohlin VOR rheometer (Bohlin Reologi, Lund, Sweden) with cone-and-plate geometry was used. The cone angle was $1^{\circ}$ and the diameter of the cone was $30 \mathrm{~mm}$. Steady-state measurements were made of the shear stress $\left(^{*}\right)$ or viscosity $(()$ of the sample as a function of shear rate $\left((\mathrm{t})\right.$ at $25^{\circ} \mathrm{C}$. The level of the strain was adjusted so that all oscillatory measurements were made within the linear viscoelastic regime. In dynamic measurement the storage $\left(G^{\prime}\right)$, loss $\left(G^{\prime}\right)$ and complex moduli $\left(G^{*}\right)$ were measured as a function of oscillation frequency. The samples were sealed with a layer of silicone oil to avoid evaporation of solvent during the measurement.

\subsubsection{Small angle X-ray scattering (SAXS)}


Small angle X-ray scattering (SAXS) measurements were performed using $\mathrm{Cu} \mathrm{K"}(8=1.542 \AA)$ radiation monochromatised with a Ni-filter and a totally reflecting glass block (Huber smallangle chamber 701). The intensity curves were measured using a linear position sensitive detector (Mbraun OED-50M). The fine focus $(0.4 \times 10 \mathrm{~mm}) \mathrm{X}$-ray tube is placed in a pointfocus position. The beam is reduced in the vertical direction with a $1 \mathrm{~mm}$ slit in front of the sample and a triangular slit in front of the detector. The $k$-range was from $0.03 \AA^{-1}$ to $0.65 \AA^{-1}$. The instrumental broadening function has fwhm $0.01 \AA^{-1}$ in the horizontal direction and $0.08 \AA^{-}$ ${ }^{1}$ in the vertical direction. Thus, the geometry is considered as point-like and, apart from the detector height and response profile, no further corrections are applied to the presented data. The background scattering was measured separately and subtracted from the intensity curves.

A small amount (ca. $20 \mathrm{mg}$ ) of the complex phase was sealed in a steel ring between $13: \mathrm{m}$ poly(imide) windows to prevent dehumidification and pressed against a hot plate (LINKAM TP93 hot stage), which has a small aperture for X-ray beam.

\subsubsection{Small angle neutron scattering (SANS)}

Small-angle neutron scattering experiments were carried out with two instruments. The first was the SANS1 instrument at the FRG1 research reactor of GKSS, Geesthacht, Germany (Shuhrmann et al., 1995). The range of scattering vectors $\left(0.008<q<0.25 \AA^{-1}\right)$ was covered by three combinations of neutron wavelengths $(8.5 \AA)$ and sample-to-detector distances $(0.7-7$ $\mathrm{m})$. The wavelength resolution was $10 \%$ (fwhm). Figure 12 shows a schematic picture of the instrumental set-up of the instrument.

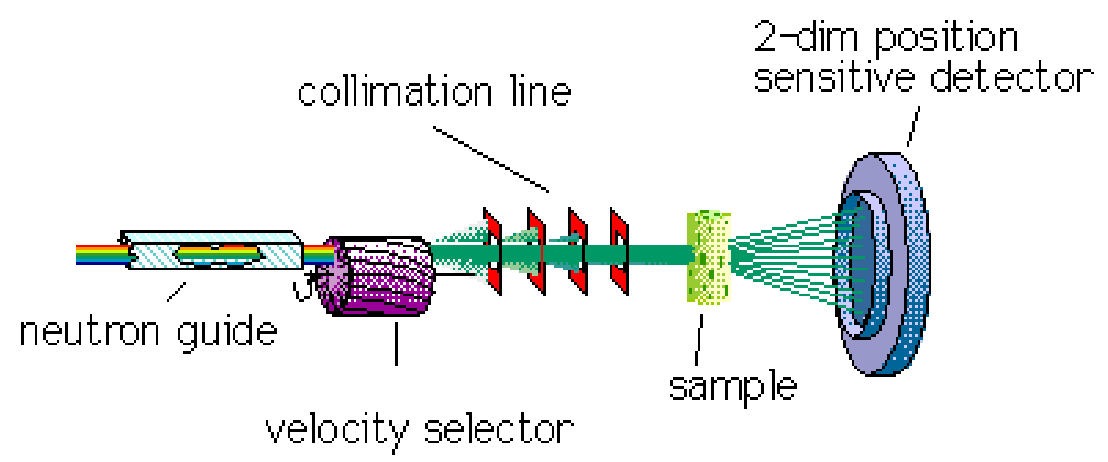

Figure 12. A schematic view of the instrumental set-up at SANS-1 at FGR-1 research reactor, GKSS. 
The second SANS instrument was the "MURN" time-of-flight small-angle neutron scattering spectrometer on the IBR-2 pulsed reactor at the Frank Laboratory of Neutron Physics, Joint Institute for Nuclear Research, Dubna, Russia (Ostanevich, 1988). The scattering vector range was $0.01<q<0.4 \AA^{-1}$, the sample-to-detector distance was $6 \mathrm{~m}$, and the neutron wavelength interval was 0.7 - $5 \AA$. The intensities, which were recorded by a one-dimensional detector, were corrected for background by subtracting the scattering of the solvent. Vanadium standard was used for calibration. The samples were kept at $70 \pm 1{ }^{\circ} \mathrm{C}$ in quartz cells with a path length of $1.5 \mathrm{~mm}$.

The raw spectra were corrected for backgrounds from the solvent, sample cell, and other sources by conventional procedures (Cotton, 1991). The two-dimensional isotropic scattering spectra were azimuthally averaged, converted to an absolute scale, and corrected for detector efficiency by dividing by the incoherent scattering spectra of pure water (Wignall and Bates, 1986), which was measured with a 1-mm-path-length quartz cell.

The data analysis included smearing appropriate to the different instrumental set-ups. For each instrumental setting, the scattering curves were smeared by the appropriate resolution function. The calculated scattering intensity was fitted to the experimental results by means of leastsquares methods (Pedersen et al., 1990). The parameters in the models were optimized by conventional least-squares analysis and the errors of parameter estimates were calculated by conventional methods (Bevington, 1969).

\subsection{Results and discussions}

\subsubsection{Critical association concentrations}

\subsubsection{Effect of the chain length of the surfactant}

The $c a c$-point ( $\log c a c$ ) decreases with increasing chain length (Fig. 13). That is also seen as an increase in the surfactant concentration, required to cause a drop in viscosity (Fig. II. 2) or a decrease in the mean particle size of the starch (Fig. II. 8). The more favourable interaction between surfactant and CS, as the surfactant chain increases, indicates, as in many other 
systems, that the critical association involves cooperative interactions between hydrocarbon chains. With Koct, neither the viscosity measurement, nor the particle sizes or surface tension measurements indicate any marked interactions between CS and surfactant. Thus, it seems that there is a minimum alkyl chain length of the surfactant required for the association to take place. For surfactants shorter than this minimum, the formation of free micelles is energetically preferred to those bound to CS.

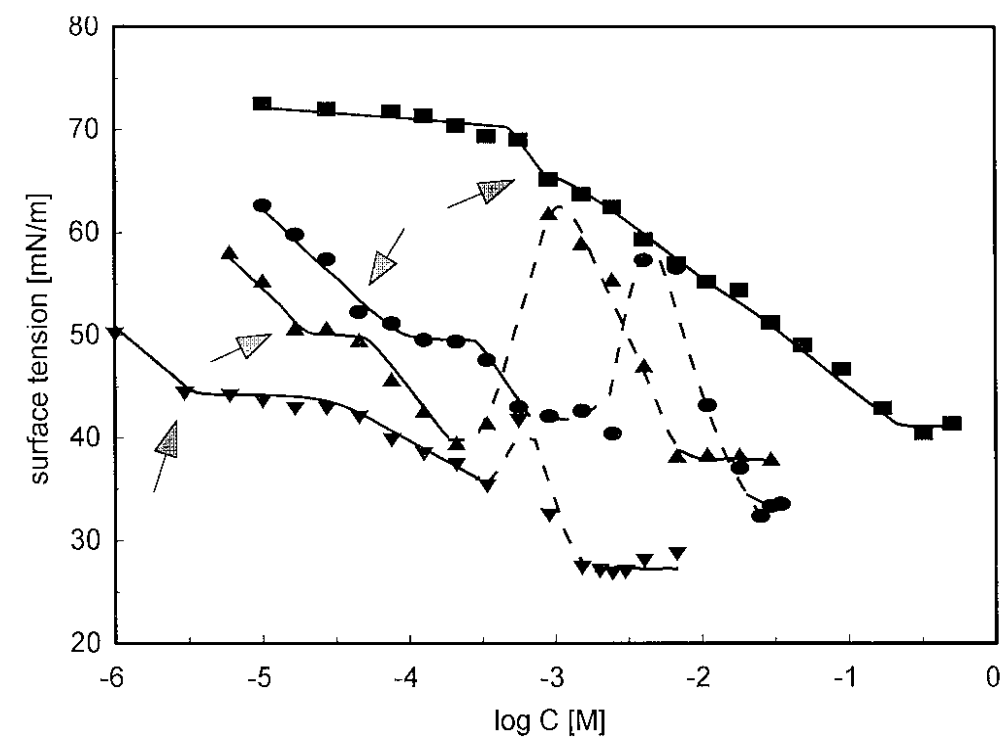

Figure 13. Surface tensions of CS/surfactant - solutions with different chain lengths having surfactants: KDod (é ), KOct (ê), NaOl (ñ ) and SDS (ï ). CS (N4) concentration was 0.01 w\%. Cac-points are indicated with arrows. The two-phase area is indicated by dashed lines.

\subsubsection{Effect of the charge density of the polymer}

The difference in the degree of substitution of CS samples has a clear effect on the cac determined by surface tension method (Fig. I. 2). The results are consistent with the notion that electrostatic interaction between CS and the surfactant increases when the linear charge density of the polymer increases. The charge density, i.e. the degree of the substitution of the CS, also has a clear effect on the viscosity of the samples. The electrostatic repulsion between charged segments increases the dimensions of the polymer molecules and leads more open conformation, which is seen from the DLS data of very low concentrations of surfactant or surfactant-free CS solutions (Fig. II. 8). 
As the surfactant concentration increases, the viscosity drops. In Figure 14, it can be seen that the lower the charge density, the lower is also the surfactant concentration that leads to a drop in the viscosity. The cacs determined from surface tensions are clearly lower than the concentrations at which the viscosity drops. Thus, this drop does not directly indicate the critical association concentration, i.e. minimum surfactant concentration required for polymer/surfactant interactions to take place. Actually, the viscosity of the solution is not a very sensitive method for detecting this kind of phenomena. It shows only what is happening with the CS particles, but is quite insensitive to the small molecule association. Thus, it seems reasonable to assume that the association of surfactants takes place first by association as counterions, which is seen on the surface tension curves (I). At somewhat higher surfactant concentrations extensive cooperative association takes place between surfactant and polymer molecules. A rapid drop in viscosity is expected when the association has reached an extent required to cause intramolecular bridging by surfactant micelles.

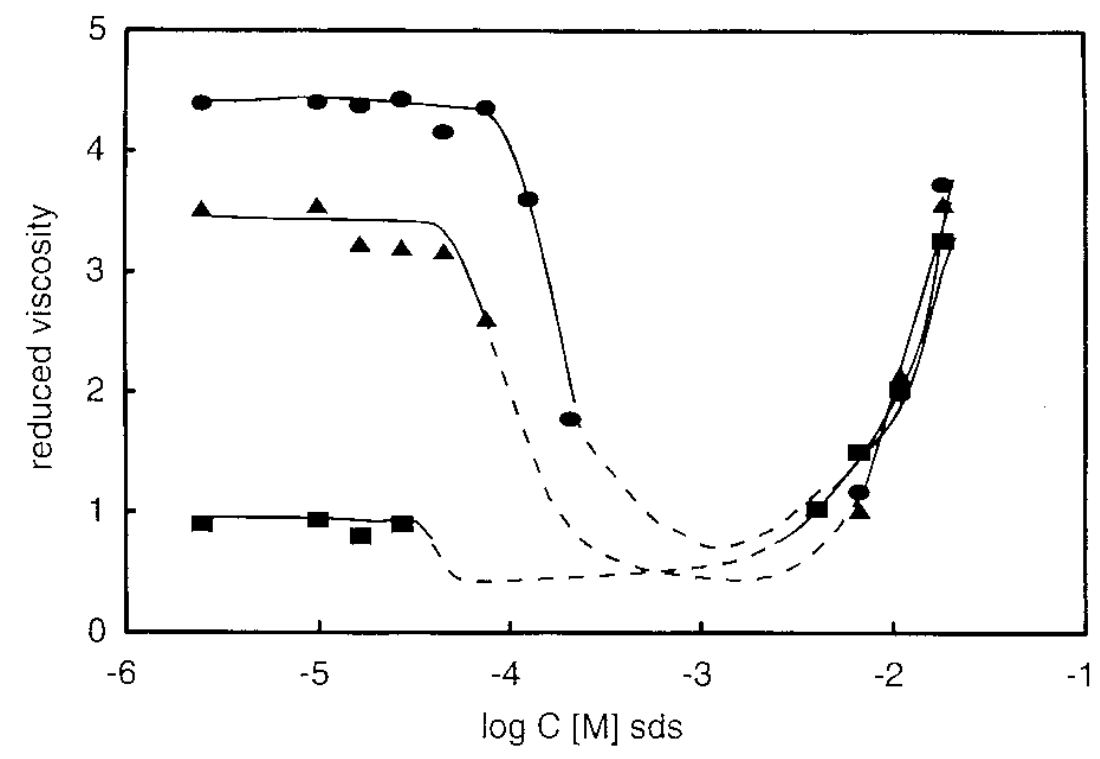

Figure 14. Reduced viscosity of CS/SDS - solutions. CS having different of degrees of substitution: 0.097 (\#), 0.415 (ï ) and 0.772 (é ). CS concentration was held constant at 0.01 $w \%$ and ionic strength $(1.3 \mathrm{mM})$. The two-phase area is indicated by dashed lines.

The electrophoretic mobilities give exactly the same information as the viscosities. The net charge of the particles begins to decrease at the same surfactant concentration as the viscosity drops (II). The size distributions (Fig. II, 10a-d) show that aggregation first takes place 
between small starch fragments and surfactant. When the charge of the CS/surfactant aggregates is almost neutral, intermolecular binding takes place and phase separation occurs.

\subsubsection{Effect of the polyelectrolyte concentration}

The polymer concentration has a large effect on the viscosity of polyelectrolyte solutions. The counterion concentration is an important parameter affecting the repulsion between charged segments on the polymer and, accordingly, the conformation of the polyelectrolyte and the viscosity of the polyelectrolyte solutions. Normally, the viscosity of polymer solution decreases when solution is diluted, but with polyelectrolytes, the situation is quite complex, because diluting the solution leads also to decreasing counterion concentration. The lower counterion concentration results in a more open polyelectrolyte conformation, thus increasing viscosity. However, as seen in Figure II, 4 the viscosity drops down at lower surfactant (SDS) concentrations the lower is the CS concentration. This stresses the importance of charge neutralization for the interactions and, above all, for the phase behaviour of CS/surfactant systems.

From surface tension measurements, it can be seen that the effect of the polymer concentration on the cac appears to be very small. The main effect is the same as that of increasing the ionic strength of the solution by adding a corresponding amount of simple electrolyte (I).

\subsubsection{Effect of electrolyte concentrations}

Adding a simple electrolyte to the solution has three effects. The main effect is screening of the electrostatic interaction between the polymer and the surfactant. Salt also affects the micelle formation because it screens the electrostatic repulsion between negative-charged head groups of the surfactant molecules, thus favouring the micelle formation. As already pointed out, the conformation of the polyelectrolyte salt is strongly affected because the repulsion between the charged units is screened. Hence, normally, in dilute concentrations adding salt increases cac a little, but at higher concentrations, the effect favouring micellisation dominates. At higher concentrations, adding salt also reduces the association and redissolution. 
From Figure 15, where different amounts of salt $(\mathrm{KCl})$ are added to CS/KDod - system, it can be observed that the association between surfactant micelles and CS coils takes place at lower concentrations when more salt is added.

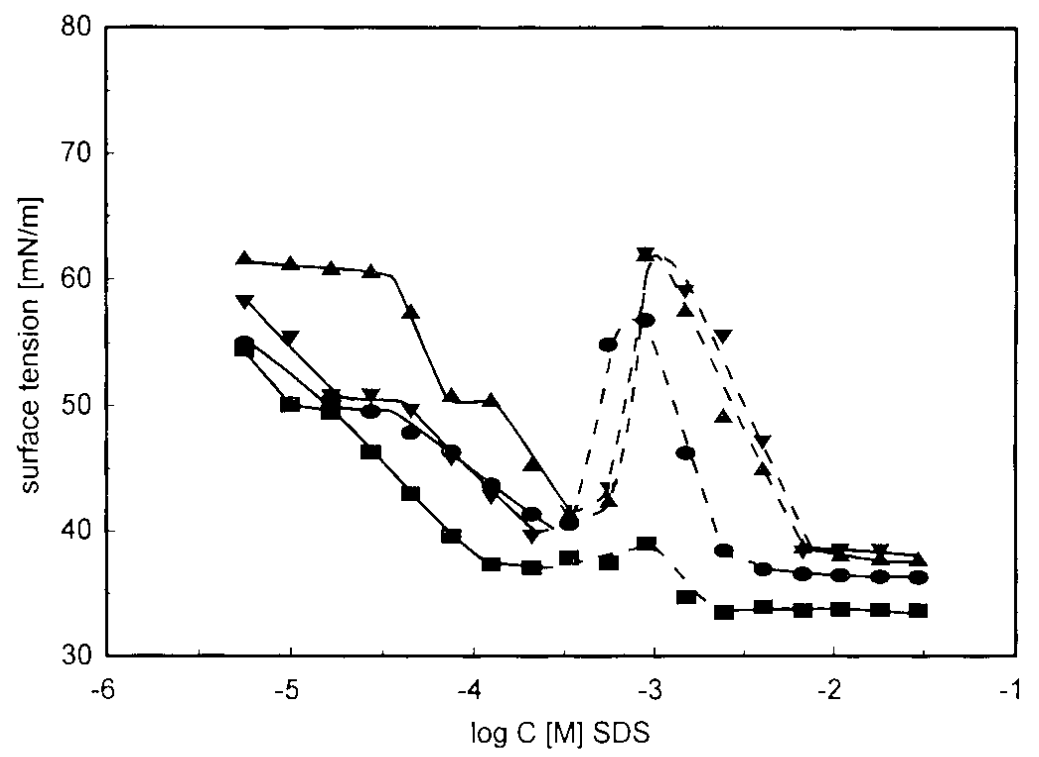

Figure 15. Surface tensions of $0.01 \mathrm{w} \% \mathrm{CS} / \mathrm{SDS}$ solutions at different concentrations of added

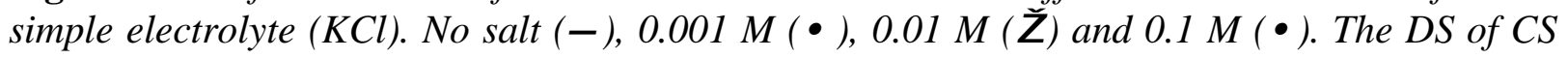
was 0.772 . The two-phase area is indicated by dashed lines.

The same effect is also seen in the viscosity curves (II, 7a and b). The other effect that is detectable, is that the difference between $c a c$ and intermolecular association (formation of gellike complex) increases with increasing electrolyte concentration (II) so also does the difference between $c a c$ and $c m c$.

\subsubsection{Phase separation and redissolution}

Phase separation is a general feature of systems of a polyelectrolyte and oppositely charged surfactant. Surfactant binding to an oppositely charged polyelectrolyte leads to reduction of the charge and hydrophilicity of the complex. Hence, phase separation is expected. The phase separation can be visually observed because the solution becomes turbid after a certain amount surfactant is added. However, the two-phase area can also be detected very sensitively in surface tension measurements, as a jump in the surface tension curve. The jump occurs because measurements are made on systems where the polyelectrolyte concentration is constant and surfactant is added; thus the investigation line crosses the tie-lines in the two-phase area so that 
the chemical potential of the components in the equilibrium phases changes as the total surfactant concentration increases. Because the surfactant is strongly bound to the polyelectrolyte, the chemical potential of the surfactant on the "surfactant-rich" side of the twophase area is lower than on the "CS-rich" side. Thus, the surfactant concentration at air/water interface decreases and surface tension increases. As excess surfactant is added, the surface tension decreases until it reaches the value that is characteristic for the pure surfactant solution above micellisation concentration (I).

It has been well proven by (Lindman et al., 1992) that phase separation is due to the aggregation of surfactant micelles and polymer coils. The results from CS/surfactant systems show that phase separation does not occur at the theoretical charge neutralisation point. Actually, it takes place when an excess of surfactant has been added and also well below the cmc of pure surfactant. When the viscosity of the solution decreases, the association between the polymer and surfactant takes place, so that the CS molecules loop around the surfactant micelles. This is clearly observed from all viscosity curves.

The viscosity rise at onset of gelation is not as big as is typical for EHEC (de Gennes, 1990, and Karlström et al., 1990) and other hydrophobic polymers (Magny et al., 1994 and Effing et al., 1994). When the gel-like complex phase is formed, because there are no hydrophobic segments on CS there is essentially no strong intermolecular network formed when the surfactant binds to the polymer. The binding is primary intramolecular. When the complex is almost neutral the complex phase forms by intermolecular association of polymer/surfactant complex.

The complex phase has very high viscosity indicating the interactions to be strong. It has also such a high degree of hydrophobia that it does not stay in the solution but separates rapidly and completely so that the viscosity of the solution drops to a minimum.

The polymer charge density has a significant effect on the phase separation. The higher the degree of substitution of $\mathrm{CS}$, the higher surfactant concentration is required to cause phase separation. Also, a certain minimum charge density on the CS is required for phase separation or other detectable interaction between polymer and surfactant to occur. With CS, sample N1, $\mathrm{DS}=0.0014$, no phase separation or other interactions were observed. 
The polymer concentration has the same kind of effect. The lower the polymer concentration, the less surfactant is needed to separate out the complex phase. Phase separation occurs clearly even in CS solution as dilute as $0.001 \mathrm{w} \%$. At high CS concentrations, phase separation occurs but it is no longer easy to detect it from surface tension curve (I). Thus, it can be assumed that both charge neutralisation and hydrocarbon chain length plays important roles in phase separation phenomena.

Increasing the surfactant chain length decreases the phase separation concentration i.e. the occurrence of phase separation runs parallel to the $c a c$ and is clearly related to cooperative interactions between the hydrocarbon chains of the surfactant. Thus, a certain minimum alkyl chain length of the surfactant is needed for the interaction. In accordance with these notions, the binding with KOct is very weak. The reason is that at the $c m c$ of short chain surfactant is high (KOct: $450 \mathrm{mM}$ ). This implies that at the $\mathrm{cmc}$ the total ionic strength of the solution is high so that, because of the weakened polycation/surfactant interactions, it is energetically more favourable to form pure surfactant micelles than polymer bound ones.

The electrolyte concentration does not affect the phase separation as markedly as in many systems of anionic polyelectrolyte and cationic surfactants (Hayakawa et al., 1983 a, b and Thalberg et al. 1991 e). Increased electrolyte concentration has a common effect in screening all electrostatic interactions. The lower boundary of phase separation decreases a little when a small amount $(1-10 \mathrm{mM})$ electrolyte is added. Higher added electrolyte concentrations $(0.1$ M), when added, clearly decrease the size of the two-phase area in the surface tension curve, but phase separation still occurs (Fig. 15 b). Thus, the interactions between CS and anionic surfactants appear to be stronger than they are in most systems of oppositely charged polyelectrolytes and surfactants. It can also be assumed that the nature of the interactions is highly cooperative due to hydrophobic interactions between alkyl chains.

\subsubsection{Dissolution of CS-surfactant precipitates}

It is commonly assumed that the dissolution of polyelectrolyte/surfactant complexes may take place by two different mechanisms. One is that redissolution results from decreased binding of the surfactant. Normally, a surfactant concentration much higher than $c m c$ is needed if redissolution is to take place by that mechanism. Another mechanism is that the increase of 
surfactant binding to such an extent that the complexes become net negatively charged, as has been reported, for example, by (Goddard and Hannan, 1976) for cationic cellulose JR400. The electrophoretic mobility of CS/surfactant aggregates was measured. The results (Fig. 16) unambiguously show that the charge of the complexes becomes negative when sufficient amount surfactant is added; at the same time redissolution takes place.

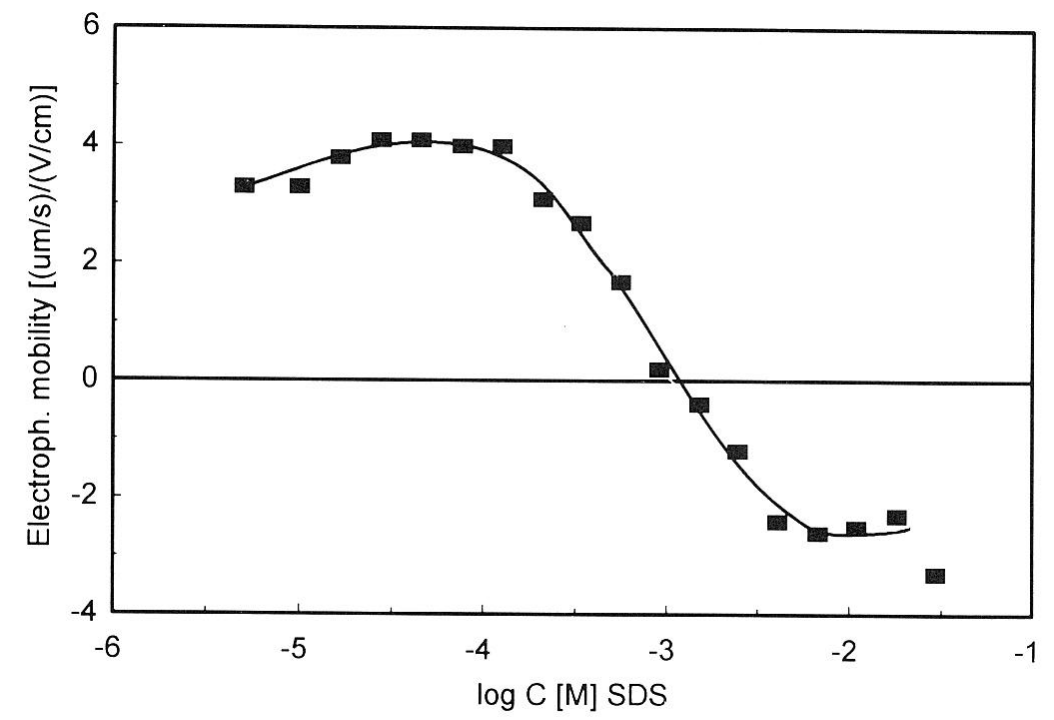

Figure 16. Electrophoretic mobility of CS/SDS particles at 298.15 K. CS (DS=0.772) concentration was $0.01 \mathrm{w} \%$.

Another fact that speaks in favour of the charge reversal mechanism is the concentration at which redissolution takes place. At low CS concentrations, it always takes place when the surfactant concentration - the $c m c$ of the pure surfactant. In the redissolution the biggest aggregates break apart, but the smallest starch fragments are left in the form of aggregates as is seen in Fig. II. 10 d. If much more surfactant than required for redissolution is added, some reaggregation takes place (II).

The charge density has some effect on the redissolution. With the highly substituted CS ( $\mathrm{DS}=0.772$ ), the redissolution is no longer complete. This is also a well-known behaviour of systems redissolving by charge reversal mechanism (Goddard and Hannan, 1977).

\subsubsection{Phase diagrams}

Three-component phase diagrams were determined for systems SDS/CS/water, KDod/CS/water and $\mathrm{NaOl} / \mathrm{CS} /$ water. The effect of adding electrolyte to these systems was also investigated. 
Actually, a four-component phase diagram would be needed to for a proper description of these systems, but the assumption that $\mathrm{NaCl}$ (or $\mathrm{KCl}$ ) forms one component with the water (aqueous solution) gives a good approximation to this type of systems. The appearance of the gel-like phase is similar to that which (Thalberg et al., 1991 b) have found in their work with PDADMAC and anionic surfactants. Although the complex nature of the cationic starch makes quantitative calculations difficult, it is obvious that the systems behave qualitatively in the same way as those described by Thalberg et al. using the modified Flory-Huggins theory of the behaviour of polymers in solution (Flory, 1969) as a model (Thalberg et al., 1990b). The surfactant has to be handled as another polyelectrolyte. This is a strong evidence for the assumption that association in CS/anionic surfactant systems happens between polyelectrolyte and associated surfactant monomers. Using this model to describe the interactions between the components leads to the following observations: the complex phase has low water content so it has high hydrophobicity, and the interaction between CS and surfactants has to be very strong when compared to systems of anionic polyelectrolytes and cationic surfactants. A general observation is that the interactions in systems of cationic polyelectrolytes/anionic surfactants interactions are stronger than in the opposite case. It has not been fully explained why it is so, but it is possible that the normally smaller head group of anionic surfactants compared with cationic surfactants plays a role. In the sample containing little more than a theoretical charge equivalent surfactant and CS, the precipitate was stiff and gel-like. When there was more or less surfactant present the precipitates were very loose.

There seems to be some differences between SDS and KDod acting as surfactants. They have both the same length of hydrocarbon chain, but the anionic group (and also the counterion) is different. The complex phase in the CS/KDod/water (Fig.17 a) system has a clearly higher water content than in the CS/SDS/water (Fig.17 b) system, indicating that the interactions between CS and SDS to be stronger than in CS/KDod system. The difference may be due the difference in interaction between substituent group in CS and anionic groups in SDS/KDod or to the difference in counterions $(\mathrm{Na} / \mathrm{K})$. 

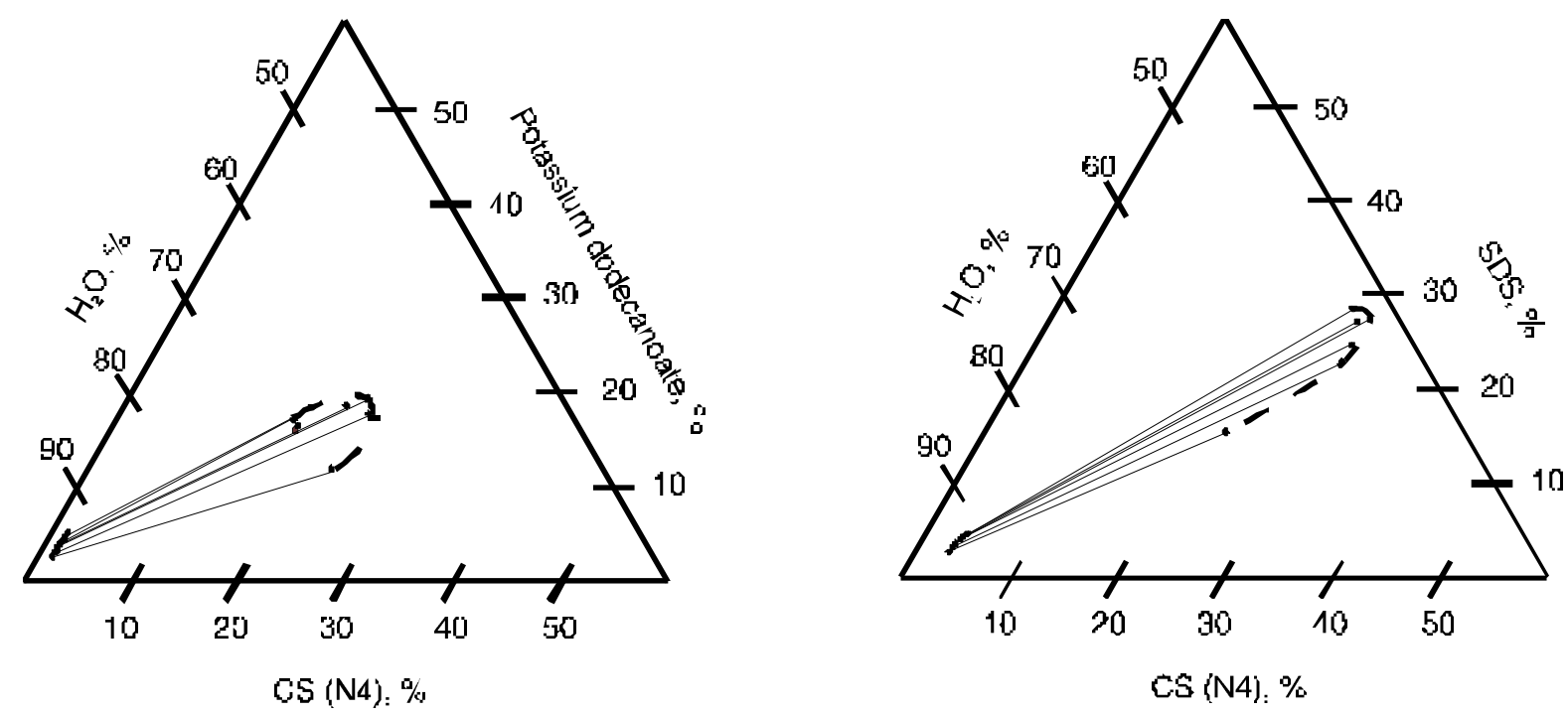

Figures 17a \& b. Partial phase diagrams of systems $C S(D S=0.772) /$ Potassium dodecanoate/water (left) and $C S(D S=0.772) / S D S /$ water (right). The lower points indicate the experimental compositions investigated; the upper dots represent analyses of the separated complex phase.

The length of the hydrocarbon chain of the surfactant seems to have a clear effect on the phase behaviour. When comparing the phase diagrams of systems containing KDod (Fig. 17 a) or $\mathrm{NaOl}$ (Fig. 18) as a surfactant there is a clear difference between the water content of the complex phase, indicating a stronger interaction with the longer chain surfactant.

The effect of added electrolyte on the consistency of the complex phase is quit weak. Comparing the phase diagram for the system of SDS/CS/0.1M NaCl (Fig. I.15), with the phase diagram of the same system without any added electrolyte, it is seen that the salt addition does not induce any significant changes in phase behaviour. This also shows quite clearly that the association behaviour of CS and anionic surfactants is highly cooperative in its nature. 


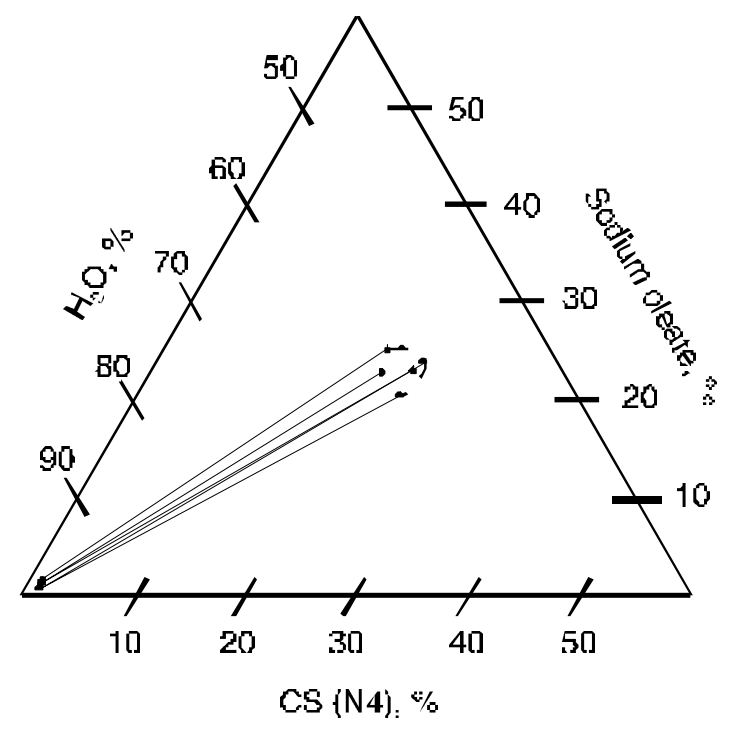

Figure 18. Partial phase diagram of the system $C S(D S=0.772) /$ sodium oleate/water at 25 " $C$. The lower points indicate the experimental compositions investigated; the upper dots represent analyses of separated complex phase.

\subsubsection{Interactions between CS and mixed anionic surfactants}

\subsubsection{Micellisation in surfactant mixtures}

Gibbs energy of formation of mixed micelles. In models of mixed micellisation (Holland and Rubingh, 1992) the Gibbs energy of mixed micelle formation is discussed in terms of several contributions (see page 6.)

In the simplest approach, it is assumed that the only contribution to the Gibbs energy of micellisation from the mixing process is the entropy of mixing of the surfactant tails. This ideal mixing model leads to a simple equation for the $c m c$ of $n$ different surfactants (Eq. 3) where mixed $c m c$ depends only on the $c m c$ s and molar ratios of the surfactants because, in ideal mixed micelles, the activity coefficient $f_{i}$ is unity. Although few micellar systems behave ideally, this model is useful as a reference system in the description of reasons for non-idealities.

Synergism in mixing of different surfactants. Mixtures of different surfactant types often exhibit synergism in the formation of micelles, i.e. deviations from ideal mixing result, in substantially 
lower $c m c$ s and interfacial tensions than would be expected based on the properties of the unmixed surfactants alone.

The most commonly used models of non-ideal mixing are based on regular solution approximation. This assumes that the excess Gibbs energy of mixing consists only of an enthalpy term, while there are no excess entropy contributions. In this model, micellar mole fractions of a binary surfactant mixture can be calculated by iterative solution provided by expression (Eq. 5). The non-ideality is characterised by a net interaction parameter $\$$, which is related to the activity coefficient (Eq. 6 and 7). From the regular solution theory it follows that in a binary surfactant system, $\$$ can be calculated from the $c m c$ s of the surfactant mixtures and the $c m c$ s of the pure components as presented in Eq. 8 .

Mixing two surfactants with same ionic head group. For binary surfactant mixtures with the same end group, a major contribution to the excess Gibbs energy of non-ideality of mixing will be due to the volume difference between the hydrophobic tails of the two surfactants. In addition to the effect on the entropy of mixing, which can be accounted for by replacing the mole fractions by volume fractions, at any given aggregation number, the mean area per molecule on the surface of the mixed micelle will be a non-linear function of the micelle composition. Another source of non-ideality is that the ionic interactions at the micelle surface will be modified. However, as a first approximation it can be assumed that the polar head group interactions will not be markedly different from those in the single surfactant micelles as long as the ionic strength is kept constant. The consequences of significant variation in the hydrophobic chain length are interesting. (Shinoda, 1954) showed that in potassium tetradecanoate/potassium octanoate mixtures the less hydrophobic potassium octanoate is almost completely excluded from the micelles.

The results confirm that micelle formation in mixed sodium alkanoate systems is close to ideal. Figure 19 shows the $c m c$ s for $\mathrm{NaOl} / \mathrm{NaDe}$ mixtures predicted by Equation (3). The system shows only a slightly negatively deviation from the ideal mixing. 


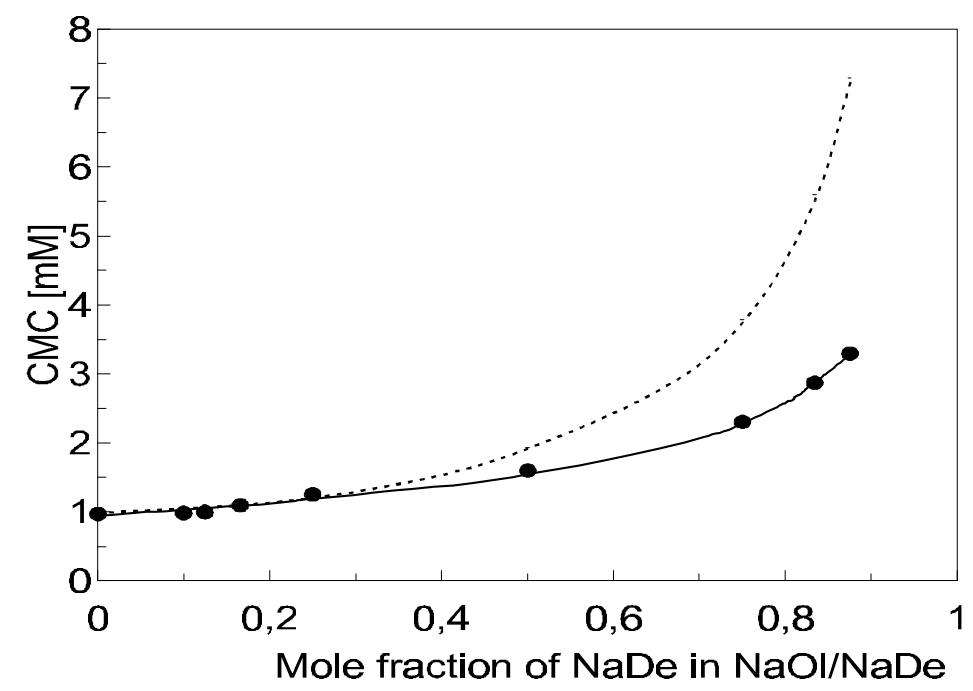

Figure 19. The $\mathrm{cmc}$ of sodium oleate $(\mathrm{NaOl}) /$ sodium decanoate $(\mathrm{NaDe})$ aqueous solution as a function of mole fraction of the short-chain surfactant (NaDe). - - - Ideal solution, (é )experimental results.

Figure IV. 9 shows the $c m c$ s of the same $\mathrm{NaOl} / \mathrm{NaDe}$ mixtures, but now the volume differences of the hydrocarbon chains of the two surfactants has been taken into account by using approximate volume fractions instead of mole fractions. The deviation from the theoretical curve, considering the free volume differences, is positive. Thus, while the calculation slightly overestimates the lowering effect of the longer-chain surfactant on the $\mathrm{cmc}$, taking the volume differences into account definitely improves the agreement between the theoretical and experimental values.

Figure 20 shows the calculated values of the interaction parameter, $\$$. The parameter, $\$$ is negative but quite small over the whole volume fraction range of $\mathrm{NaDe}$. Thus, it can be asserted that the chain volume differences between $\mathrm{NaOl}$ and $\mathrm{NaDe}$ are sufficient to almost completely explain the deviations of the surfactant mixture in the micelles from ideality. 


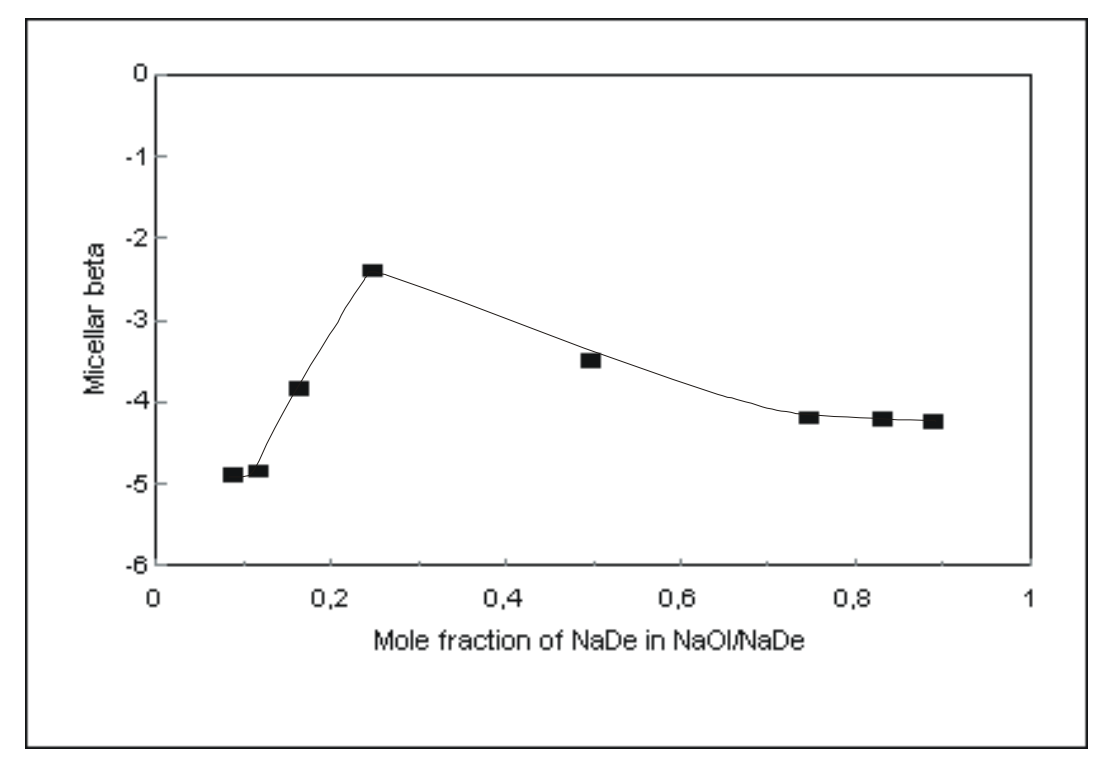

Figure 20. The unideal interaction parameter, $\$_{2}$, in NaOl/NaDe solutions as a function of the mole fraction of $\mathrm{NaDe}$.

\section{3. 4. 2. Critical association concentrations}

Association with cationic starch. It has been shown in articles I and II that the main interactions controlling the CS/surfactant association are the hydrophobic interactions between the hydrocarbon chains of the surfactant, and the electrostatic interactions between the cationic starch and the anionic micelles. When another surfactant is added to the system the packing conditions of the monomers in the micellar aggregates are changed. Changing the mean size of the hydrophobic tail changes the balance between hydrophobic interactions and electrostatic repulsion.

When the fraction of the less hydrophobic surfactant increases the cac also increases. This is analogous to the behaviour of single surfactant/CS systems, in which the cac increases when the surfactant chain length decreases. This association is reflected in the dependence of surface tension and viscosity on surfactant concentration as a break point in the surface tension, and in a sudden drop in the reduced viscosity.

In the CS/mixed surfactant systems, the cac markedly increases when the chain length of the second surfactant decreases in the order $\mathrm{NaDod}<\mathrm{NaDe}<\mathrm{NaOct}$ (Figures IV. 4 and IV. 5). The mean degree of hydrophobia of the surfactant mixture is the lower the shorter is the chain 
length of the second surfactant and, in analogy with the formation of free mixed micelles, micellisation on the polymer chains occurs at higher concentrations (table IV.2).

Polymer/surfactant mixture interaction parameter. Polymer/surfactant association can be described as a polymer induced micellisation. Thus, there is an obvious analogy between mixed micelle formation in pure surfactant systems and the cooperative formation of surfactant aggregates on the polymer in systems of surfactant mixtures and polymers. To illustrate this, we assume that the electrostatic interactions are independent of micellar composition, and that mixed micelle formation on the polycation is completely analogous to the formation of free micelles, except that the presence of the polycation strongly reduces the repulsive electrostatic interactions between the ionic end groups. Then a specific interaction parameter, $\$_{c a c}$, can be calculated for the mixed micelles associated with the polymer. In an analogy to the interaction parameter for free mixed micelles, by substituting the $c a c$ s for the $c m c$ in Eq. (8):

$$
\beta_{C A C}=\frac{\ln \left[\alpha_{1} C A C_{m i x}^{*} /\left(x_{1} C A C_{1}^{*}\right)\right]}{\left(1-x_{1}\right)^{2}}=\frac{\left\{\alpha_{2} C A C_{m i x}^{*} /\left[\left(1-x_{1}\right) C A C_{2}^{*}\right]\right\}}{x_{1}^{2}}
$$

where $C A C_{1}{ }^{*}$ is the $c a c$ with surfactant $1, C A C_{2}{ }^{*}$ is the $c a c$ with surfactant 2 and $C A C_{M i c}{ }^{*}$ is the $c a c$ in the surfactant mixture. The values of the specific interaction parameter, $\$$ cac , can be used as a measure of the synergism in polymer/binary surfactant systems. Figure 21 shows $\$_{c a c}$, as a function of mole fraction of $\mathrm{NaDe}$ in $\mathrm{CS} / \mathrm{NaOl} / \mathrm{NaDe}$ system. There is no synergism observed in polymer/surfactant association in the sense that there would be a maximum in the value of $\$$ cac The interaction parameter is small and changes from negative to positive when the fraction of the short-chain component increases. Thus, mixed micelle formation with the polymer also seems to result in the formation of a remarkably ideal surfactant mixture in the micelles. 


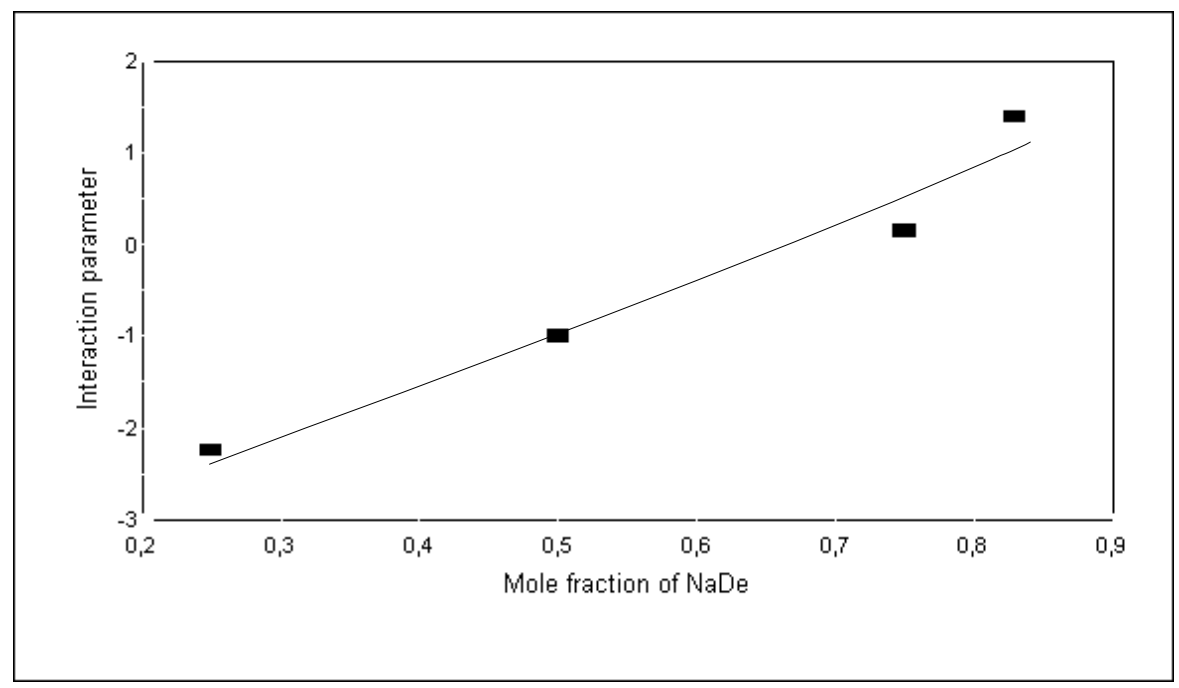

Figure 21. Calculated polymer/binary surfactant interaction parameter, $\$_{c a c}$, as a function of the mole fraction of NaDe of the total surfactant concentration in CS (DS=0.80, $\mathrm{C}=0.01 \mathrm{w} \%) / \mathrm{NaDe} / \mathrm{NaOl}$ system.

\subsubsection{Phase separation and redissolution}

Phase separation is a general feature of aqueous systems of a polyelectrolyte and an oppositely charged surfactant. The binding of surfactant results in a complex with a lower charge and which is less hydrophilic than the initial polymer. When the amounts of the polymer and surfactant are roughly charge-equivalent, the complex precipitates as a water-rich gel. It is by now well established that phase separation is due to the association of surfactant micelles (in this case more precisely mixed micelles) with polymer coils. As is reported in article I, phase separation takes place at surfactant concentration somewhat higher than the theoretical chargeneutralization point but well below the $c m c$ of the pure surfactant. When an excess of surfactant is added the complex partly redissolves. The redissolution takes place trough a charge reversal mechanism (Hayakawa et al., 1991).

Figures IV. 3-5 show that the higher the mole fraction of the short-chain component in the surfactant mixture, the higher the concentration at which phase separation takes place. This is not surprising, because when the concentration of short-chain surfactant increases, the fraction of this surfactant in the micelles increases and, hence, their hydrophobicity decreases. As expected, redissolution also takes place at higher concentrations when the fraction of the shortchain surfactant increases. 
At a given bulk composition, the chain length of the shorter surfactant does not affect the surfactant/polymer ratio in the complex phase, but the maximum water content of the complex increases strongly when the chain length of the shorter chain surfactant decreases (Figure 22). This indicates reduced hydrophilicity of the complex. Redissolution of the complexes takes place at somewhat lower surfactant concentrations when the chain length of the shorter surfactant decreases.

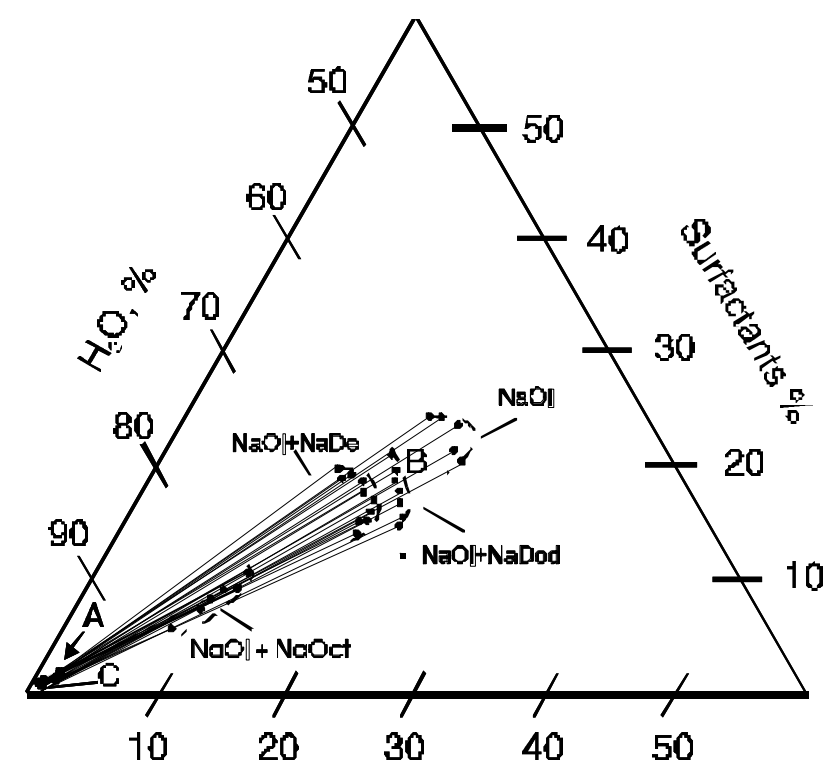

Figure 22. Partial phase diagram of the system of CS $(D S=0.80) / \mathrm{NaOl} /$ second surfactant/water at $25^{\circ} \mathrm{C}$. The surfactant mixtures are: NaOl; NaOl/NaDod; NaOl/NaDe and NaOl/NaOct. The molar ratio of the surfactants in mixtures is 1:1. Points A indicate the compositions prepared in experiments; points $B$ gives analysed compositions of the complex phase and points $C$ represent the compositions of the supernatant aqueous phase.

\section{3. 4. 4. Surfactant ratio in CS/mixed micelle complex phase}

The molar ratio of the surfactants in mixed micelles depends on their chain length difference. For a given bulk composition, free mixed micelles contain less short-chain surfactant, the larger the chain length difference. The same behaviour is observed in the CS/surfactant complexes. (Figure IV. 8). Thus, the fraction of $\mathrm{NaDe}$ in $\mathrm{CS} / \mathrm{NaOl}+\mathrm{NaDe}$, when the molar ratio of surfactant monomers is 1 to 1 , is only about 0.003 . In systems of $\mathrm{CS} / \mathrm{NaOl}+\mathrm{NaDod}$ where the chain length difference of the surfactants is not so large, the corresponding fraction of the 
NaDod is about 0.009. This again stresses that the association between CS and surfactants can be understood as a polycation enhanced micellisation of the surfactants on the polymer.

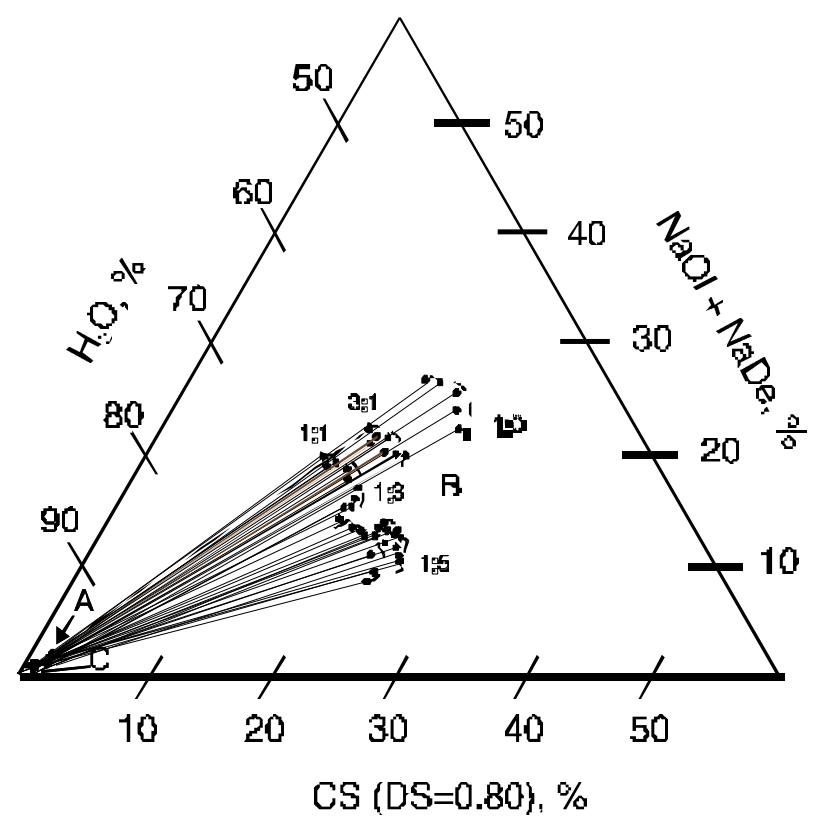

Figure 23. Partial phase diagram of the system of CS $(\mathrm{DS}=0.80) / \mathrm{NaOl} / \mathrm{NaDe} /$ water at $25^{\circ} \mathrm{C}$. The ratios of $\mathrm{NaOl} / \mathrm{NaDe}$ are 3:1, 1:0, 1:1, 1:3 and 1:5. Points $A$ indicate the compositions prepared in experiments; points $B$ gives analysed compositions of the complex phase and points $C$ represent the compositions of the supernatant aqueous phase.

A consequence of this behaviour is that the bulk molar ratio of the surfactants does not strongly effect on the molar ratio of the surfactants in the aggregate phase. When the bulk fraction of the short-chain surfactant varies from 0.2 to 0.9 , its fraction in complex phase stays almost constant at 0.01 . This implies that the long-chain surfactant $(\mathrm{NaOl})$ associates almost totally with cationic starch when the fraction of the short-chain surfactant is high enough.

\subsubsection{Structure of CS-surfactant systems in dilute solutions}

Figure 23 shows the differential cross section of SANS from solutions of single surfactants in heavy water as well as the curves fitted using Eq. (48). Note that the concentration is different for the different surfactants. Different concentrations were used because the $c m c$ s of the used surfactants ranged from $\sim 1$ to $\sim 450 \mathrm{mmol} \mathrm{dm}^{-3}$, and the solubility of the surfactant with the longest hydrocarbon chain length is quite low at reasonable temperatures. The intensity increases and the position of the interference maximum shifts to lower $q$ values when the alkyl 
chain length increases. This shows that larger micelles are formed by surfactants with longer alkyl chains, as is well known from earlier studies (Chevalier and Zemb, 1990). Agreement between theoretical curves and experimental data is excellent, i.e. the model of micelles as prolate rotational ellipsoids or spheres interacting through a screened Coulomb potential satisfactorily describes the experimental data. The small deviation at the lowest $q$ range is probably due to the limitations of instrumental resolution.

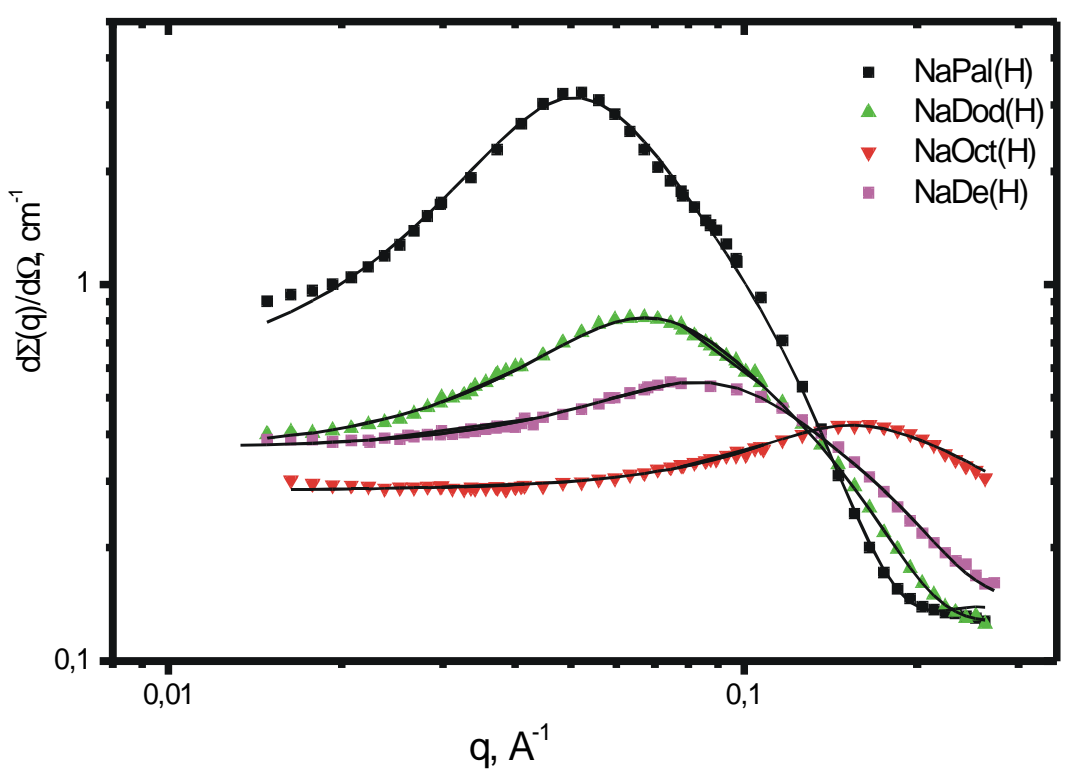

Figure 23. The differential cross-section of SANS from solutions of single surfactants (nondeuterated) in heavy water as well as the curves fitted using Eq. (48). Surfactant concentrations: NaPal and NaDod, $100 \mathrm{mM}$; NaDe, $200 \mathrm{mM}$; NaOct, $600 \mathrm{mM} . \mathrm{T}=343 \mathrm{~K}$.

Parameters obtained by the fitting procedure are given in Table VI. 2. When the chain of the surfactant becomes shorter, the average diameter of the micelles decreases from $71 \pm 2 \AA$ (NaPal) to $31 \pm 5 \AA$ (NaOct) with simultaneous change in the micellar shape from prolate ellipsoid with axis ratio $2.0 \pm 0.2$ ( NaPal) to spherical ( $\mathrm{NaDe}$ and $\mathrm{NaOct})$. The degree of dissociation increases in the order $\mathrm{NaPal}<\mathrm{NaDod}<\mathrm{NaDe}$, which agrees with many other observations of the dependence of $\alpha$ on chain length. The lower value of $\alpha$ for NaOct is probably due to experimental inaccuracy.

\subsubsection{Mixed micelles}


Solutions of $\mathrm{NaPal}(\mathrm{H}) / \mathrm{NaDod}(\mathrm{H}), \mathrm{NaPal}(\mathrm{H}) / \mathrm{NaDe}(\mathrm{H})$ and $\mathrm{NaPal}(\mathrm{H}) / \mathrm{NaOct}(\mathrm{H})$ in heavy water were investigated (molar ratio 1:1, $(\mathrm{H})$ indicates hydrogen-based surfactants). The results were compared to measurements on similar solutions of $\mathrm{NaPal}(\mathrm{D}) / \mathrm{NaDod}(\mathrm{H}), \mathrm{NaPal}(\mathrm{D}) / \mathrm{NaDe}(\mathrm{H})$ and $\mathrm{NaPal}(\mathrm{D}) / \mathrm{NaOct}(\mathrm{H})$, where (D) denotes deuteration. The total surfactant concentration was $100 \mathrm{mM}$, which is above the $c m c$ of pure NaPal and NaDod but below the $c m c$ of $\mathrm{NaDe}$ and $\mathrm{NaOct}$. The results are shown in Figures $24 \mathrm{a}$ and $\mathrm{b}$.

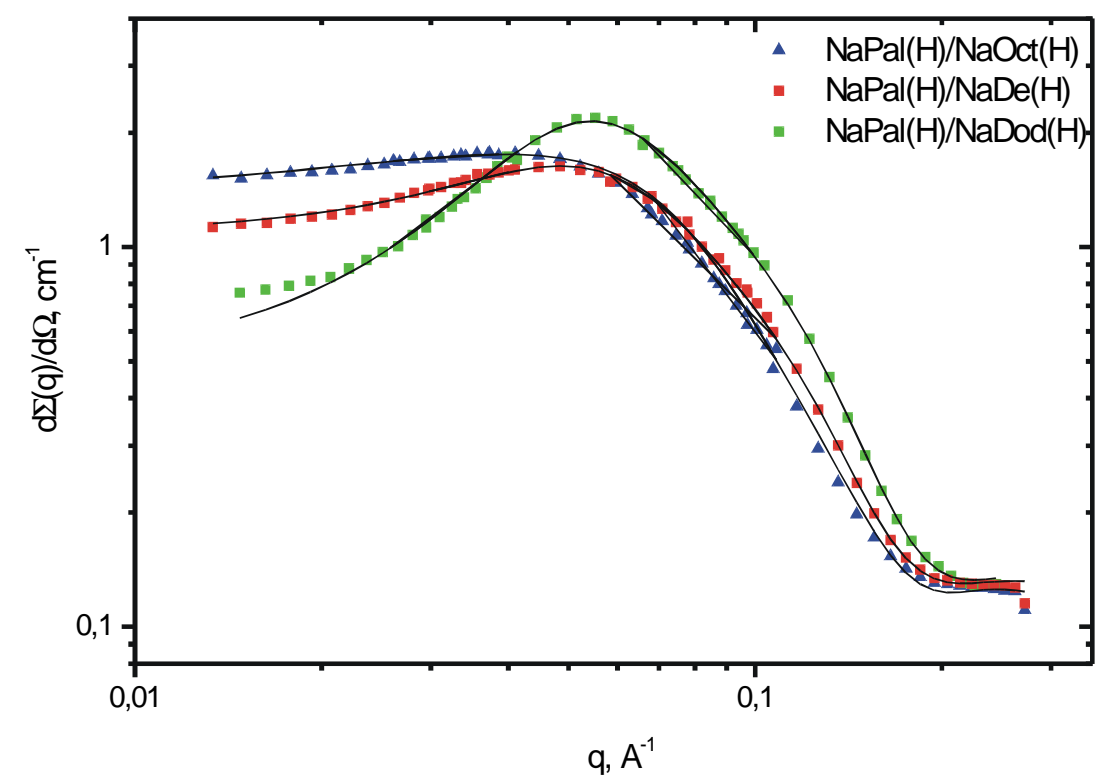

Figure 24.a. The differential cross section of SANS from solutions of 1:1 surfactant mixtures (non-deuterated) in heavy water as well as curves fitted using Eq. (53). Total surfactant concentrations $100 \mathrm{mM}$.

Marked formation of mixed micelles is detected only in the NaPal/NaDod system. Due to the large difference in the scattering-length densities, the ratio of the scattering intensity from $\mathrm{NaPal}(\mathrm{H})$ in $\mathrm{D}_{2} \mathrm{O}$ to the scattering from $\mathrm{NaPal}(\mathrm{D})$ in $\mathrm{D}_{2} \mathrm{O}$ is $\sim 100$. It is not possible to detect pure $\mathrm{NaPal}(\mathrm{D})$ micelles in $\mathrm{D}_{2} \mathrm{O}$ by SANS. $q$ at the interference maximum is equal for $\mathrm{NaPal}(\mathrm{H}) / \mathrm{NaDod}(\mathrm{H})$ and $\mathrm{NaPal}(\mathrm{D}) / \mathrm{NaDod}(\mathrm{H})$, which indicates that the concentration of micelles is the same in both solutions.

From the intensity ratio of $\mathrm{NaPal}(\mathrm{D}) / \mathrm{NaDod}(\mathrm{H})$ to $\mathrm{NaPal}(\mathrm{H}) / \mathrm{NaDod}(\mathrm{H})$ it can be estimated (Holland and Rubingh, 1992) that the fraction of NaPal molecules in the mixed micelle is $0.49 \pm$ 0.02. Thus, the composition of the mixed micelles is the same as the total composition of surfactant in solution. This result confirms that at concentrations much higher than the $c m c$ of a surfactant mixture, the composition of the micelles is approximately the same as the total composition of the surfactant in solution (Pedersen et al., 1990). 


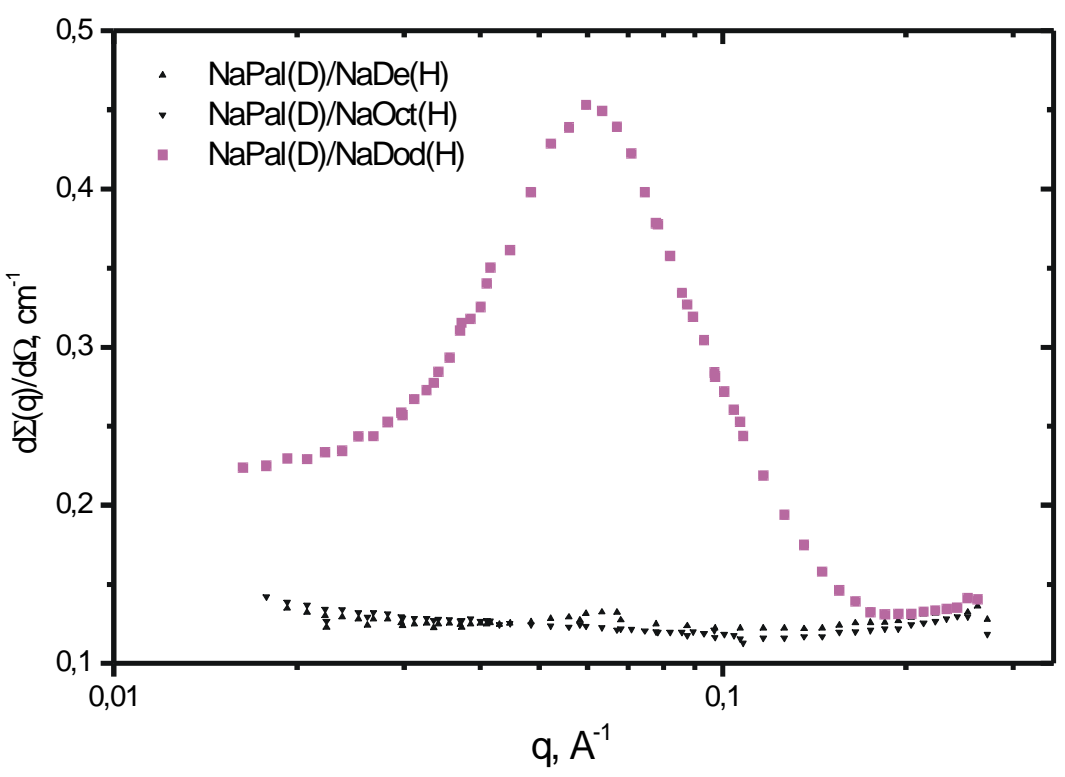

Figure 24.b. The differential cross-section of SANS from solutions of 1:1 mixtures of deuterated NaPal and non-deuterated surfactants with shorter chains in heavy water as well as curves fitted using Eq. (53). Total surfactant concentrations $100 \mathrm{mM}$.

There is no sign of scattering in the curves for $\mathrm{NaPal}(\mathrm{D}) / \mathrm{NaOct}(\mathrm{H})$ and $\mathrm{NaPal}(\mathrm{D}) / \mathrm{NaDe}(\mathrm{H})$ (Figure 24b). This indicates that in $100 \mathrm{mM} \mathrm{NaPal} / \mathrm{NaDe}$ and $100 \mathrm{mM} \mathrm{NaPal} / \mathrm{NaOct}$ only pure $\mathrm{NaPal}$ micelles are formed. However, the size and interactions of the micelles are affected different ways by $\mathrm{NaDe}$ and $\mathrm{NaOct}$.

Addition of NaDe increases the effective micellar charge. The effect leads to significant intermicellar interactions, as shown by the maximum in the scattering intensity. Thus, it seems that $\mathrm{NaDe}$ is to some extent incorporated in the micelles. Presumably, because the total concentration is below the $c m c$ of pure NaDe, very small amounts of the surfactant will be incorporated so that the micelles cannot be detected by SANS when the NaPal contrast is low.

In the model calculations (drawn lines in Fig. 24a) it was assumed that the micelles in $\mathrm{NaPal}(\mathrm{H}) / \mathrm{NaDe}(\mathrm{H})$ and $\mathrm{NaPal}(\mathrm{H}) / \mathrm{NaOct}(\mathrm{H})$ contain only $\mathrm{NaPal}$ and that the $\mathrm{NaPal} / \mathrm{NaDod}$ ratio in the micelles is 1:1, i.e. the same as in bulk solution. The agreement between theoretical curves and experimental data is as good as for single surfactant solutions. The dimensions of the mixed $\mathrm{NaPal}(\mathrm{H}) / \mathrm{NaDod}(\mathrm{H})$ micelles in 1:1 solutions lie between those of pure $\mathrm{NaPal}$ and pure NaDod (Table VI.3). The surface charge is closer to the charge of pure NaDod micelles.

When the same fitting procedure was applied to the data for $\mathrm{NaPal}(\mathrm{D}) / \mathrm{NaDod}(\mathrm{H}), N_{a}$ turned out to be about $10 \%$ smaller than for $\mathrm{NaPal}(\mathrm{H}) / \mathrm{NaDod}(\mathrm{H})$. The degree of dissociation is about the same and the axial ratio is only 1.0. A possible explanation is that the NaPal and NaDod 
molecules are not evenly distributed in the prolate micelles. The radius of the cylindrical part of the prolate micelle is clearly closer to the chain length of the NaDod than to that of NaPal. This implies that the middle part of the micelle must be enriched in NaDod, with NaPal located close to the end caps. Accordingly, SANS detects an aggregate with lower axial ratio in the $\mathrm{NaPal}(\mathrm{D}) / \mathrm{NaDod}(\mathrm{H})$ system, where only $\mathrm{NaDod}$ contributes to the scattering. It is not quite clear why this situation should be energetically favourable, as the mean radius of curvature is smaller in end caps, and the bending energy of a mixed surfactant bilayer should be lower, the higher the fraction of short-chain surfactant (Barneveld et al., 1992, 1994).

The results from fitting the theoretical curves to the results from $\mathrm{NaPal}(\mathrm{H}) / \mathrm{NaDe}(\mathrm{H})$ and $\mathrm{NaPal}(\mathrm{H}) / \mathrm{NaOct}(\mathrm{H})$ solutions confirm that the effects of $\mathrm{NaDe}$ and $\mathrm{NaOct}$ on $\mathrm{NaPal}$ micelles are different.

The micelles in the $\mathrm{NaPal} / \mathrm{NaOct}$ solution are larger than the micelles in $\mathrm{NaPal} / \mathrm{NaDe}$ or $\mathrm{NaPal}$ solutions (Tables VI.2 and 3). The micelles consist almost entirely of NaPal because the concentration of NaOct is far below its $\mathrm{cmc}$, so that it should have little tendency to participate in micelle formation. Thus, the effect of NaOct is mainly that of a simple electrolyte that promotes micelle formation by better screening of electrostatic repulsion The result is that the micelles formed by NaPal grow slightly, the degree of dissociation increases slightly, intermicellar interactions decrease, and the interference maximum almost disappears.

The micellar aggregation number in $100 \mathrm{mM} \mathrm{NaPal} / \mathrm{NaDe}$ is lower than in $100 \mathrm{mM}$ pure NaPal, while the degree of dissociation increases. The smaller size could be due to the lower concentration of $\mathrm{NaPal}$ in the mixed system, but the higher charge and the fact that NaDe does not affect micellar properties in the same way as NaOct indicate that $\mathrm{NaDe}$ does participate in some way in micelle formation, although this cannot be directly detected by SANS. $50 \mathrm{mM}$ $\mathrm{NaDe}$ is much closer to its $\mathrm{cmc}$ than $50 \mathrm{mM} \mathrm{NaOct,} \mathrm{so} \mathrm{this} \mathrm{conclusion} \mathrm{does} \mathrm{not} \mathrm{seem}$ unreasonable.

Figure 25 shows the dependence of the aggregation number of mixed NaPal/NaDod micelles on the mean chain length of the surfactant at constant surfactant concentration (100 mM). 


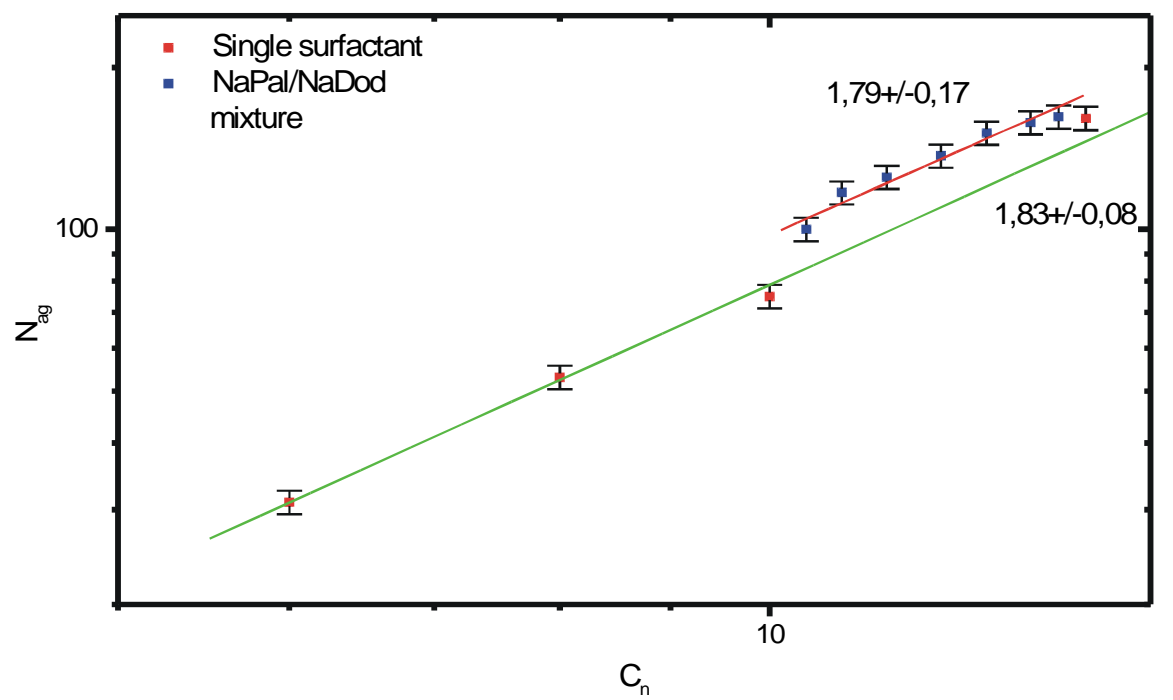

Figure 25. The mean aggregation number of mixed NaPal/NaDod micelles as a function of the mean chain length of the surfactant. The carbon number means the number of $\mathrm{CH}_{2}$-units in the alkyl chain. As shown are the aggregation numbers of pure single chain surfactants.

In the evaluation of aggregation numbers it was assumed that the composition of the micelles is the same as the bulk composition of surfactant. The size of both the mixed micelles and the single surfactants grow at about the same rate when the alkyl chain length increases. However, the values of $N_{a}$ of the mixed micelles are slightly larger than those inferred from the values of single surfactants by linear interpolation. Indeed, some synergism in the formation of mixed micelles by surfactants with different alkyl chain is to be expected (Barneveld et al., 1994), as is also shown by the values of the $c m c$ s for mixtures (table VI.1).

\subsubsection{CS/surfactant complexes}

The cationised and depolymerised starch used in these studies has $M_{w}<10^{6}$ which implies that it consists of molecules of the same size as native potato amylose. It carries a high charge due to the quaternary ammonium substituents. This strengthens the interaction between CS and oppositely charged surfactants. The conventional theory of polymer-surfactant association assumes that the surfactant monomers are associating with the polymer chain as micelles or micelle-like aggregates. As will be shown below, the helical conformation of the cationic starch seems to result in a different type of structure, i.e. the formation of inclusion complexes.

Figure VI.4 shows SANS from a $0.8 \%$ solution of $\mathrm{CS}$ in $\mathrm{D}_{2} \mathrm{O}$ to which has been added different concentrations of surfactant $(\mathrm{NaPal})$. The compositions were chosen so that the scattering from CS could be separated from the scattering of CS/surfactant aggregates. Thus, CS Isurfactant 
aggregates should be seen with the combination $\mathrm{CS} / \mathrm{NaPal}(\mathrm{H}) / \mathrm{D}_{2} \mathrm{O}$ while only $\mathrm{CS}$ should be seen with the combination $\mathrm{CS} /$ surfactant(D)/ $\mathrm{D}_{2} \mathrm{O}$. NaPal was chosen because it forms the largest micelles of the investigated surfactants.

The differential scattering intensities immediately indicate that two processes take place in the system as the surfactant concentration increases: on the one hand the formation and growth of CS/surfactant complexes and, on the other hand, changes in the conformation of CS. The scattering at low values of $q$ suggests that the overall size of the aggregates is larger compared with the experimental interval of scattering vectors so that the scattering cross sections observed are essentially due to intra-particle interactions. For this reason, and because the CS neutralizes the charge of the surfactant aggregates, inter-particle interactions were neglected in the analysis of scattering data.

In Figure VI.5 is shown differential SANS cross-sections for CS/NaPal(H), $\mathrm{CS} / \mathrm{NaPal}(\mathrm{H}) / \mathrm{NaDod}(\mathrm{H})$ and $\mathrm{CS} / \mathrm{NaDod}(\mathrm{H})$ mixtures. The concentration of $\mathrm{CS}$ and the total surfactant concentration is the same in all systems $(0.8 \%$ and $8 \mathrm{mM}$, respectively). The surfactant concentration is much higher than the critical association concentration of $\mathrm{CS} / \mathrm{NaPal}$ ( $c a c \sim 0.01 \mathrm{mM}$ ) so that significant amounts of CS/surfactant complexes should be present. The scattering intensities indicate that the largest complexes occur in the $\mathrm{CS} / \mathrm{NaPal}$ system and that the difference between $\mathrm{CS} / \mathrm{NaPal}$ and $\mathrm{CS} / \mathrm{NaPal} / \mathrm{NaDod}$ complexes is smaller than the difference between $\mathrm{CS} / \mathrm{NaPal} / \mathrm{NaDod}$ and $\mathrm{CS} / \mathrm{NaDod}$ complexes. Apparently, $\mathrm{NaPal}$ is enriched in the complex phase formed by the $\mathrm{NaPal} / \mathrm{NaDod}$ mixtures.

When interparticle interactions are neglected the differential scattering cross-section can be written as

$$
\frac{d \sum(q)}{d \Omega}=\frac{d \sum(0)}{d \Omega} p^{2}(q)+B_{i n c}
$$

where $d \Sigma(0) / d \Omega$ is the scattering at "zero" angle and $B_{i n c}$ is the incoherent scattering background. $d \Sigma(0) / d \Omega$ is related to the concentration of particles $\left(N_{v}\right)$, volume $(V)$ and the scattering length densities by 


$$
\frac{d \sum(0)}{d \Omega}=N_{v}\left(<\rho>-\rho_{s}\right)^{2} V^{2}
$$

where $\langle\rho\rangle$ is the average scattering length density of the particle. The slope of a log/log plot of the differential cross-section vs. $q$ is close to -1 , which indicates that elongated structures are formed. Therefore, in the analysis of experimental data, it is assumed that the aggregates are cylindrical. In this case the single-particle scattering function $P^{2}(q)$ is equal to $\left\langle F^{2}(g)\right\rangle$ where $F(q)$, the form factor of a cylinder is written as

$$
F(q)=\int_{0}^{\pi / 2}\left[\frac{\sin (q L / 2 \cos \beta) 2 J_{1}(q R \sin \beta}{(q L / 2 \cos \beta)(q R \sin \beta)}\right]^{2} \sin \beta d \beta
$$

where $L$ is the length and $R$ is the radius of the cylinder, $J_{1}$ is the first-order Bessel function, and $\beta$ is the angle between the $q$ vector and the axis of the cylinder.

Four parameters were varied in the fitting of theoretical differential cross-sections to experimental results: $R, L, d \Sigma(0) / d \Omega$ and $B_{\text {inc }}$. The model describes the experimental data for $q$ values larger than $0.01 \AA^{-1}$. The deviations at lower $q$ could be due to the presence of some much larger particles (possibly some incompletely dissolved CS). Parameters obtained by the fitting procedure are given in table VI. 4.

The first row in table VI.1 shows that pure CS in solution exhibits local cylindrical behaviour (radius of cylinder $30 \AA$ ). Probably the pure CS solution contains cylinder-like aggregates in the size-range investigated. The cylinder length is $\sim 600 \AA$ In the $q$ interval used it is difficult to estimate this parameter more accurately, but this is a very reasonable value for starch with the molecular weight of the CS, assuming that it has a helical conformation.

The radius of the cylindrical CS-surfactant aggregates decreases when the surfactant concentration increases. This result is expected, because when surfactant associates with the CS, the repulsion between cationic substituent-groups decreases so that the conformation of the CS molecules becomes more compact. Hydrophobic interactions between the surfactant chains may also contribute. 
When deuterated surfactants are used (Figure VI.6), the intensities are only about a tenth of the intensities, recorded for protonated surfactants. They fall in the same range as for cationic starch without surfactant (Figures VI.2 and 4). However, the dependence of the scattering crosssection on $q^{-1}$ still indicates the formation of cylindrical aggregates. This confirms that fraction of surfactants in the CS/surfactant complexes is high. The scattering of mixed $\mathrm{NaDod}(\mathrm{D}) / \mathrm{NaPal}(\mathrm{D}) / \mathrm{CS}$ aggregates is lower than for pure $\mathrm{NaDod}(\mathrm{D}) / \mathrm{CS}$ or $\mathrm{NaPal}(\mathrm{D}) / \mathrm{CS}$. The reason for this is not clear; it may be an experimental artefact.

In the analysis of surfactant systems, the length of cylinder was kept constant and only its radius was adjusted. The cylinder radius of deuterated complexes is larger than the radius obtained for fully protonated complexes. This suggests that the surfactant molecules are inside the CS/surfactant complexes with the CS molecules forming some kind of shell around the surfactant aggregates. Due to the low intensity of scattering from CS, the contribution of CS shell scattering is less visible in $\mathrm{CS} / \mathrm{NaPal}(\mathrm{H}), \mathrm{CS} / \mathrm{NaDod}(\mathrm{H})$ and $\mathrm{CS} / \mathrm{NaPal}(\mathrm{H}) / \mathrm{NaDod}(\mathrm{H})$ mixtures.

The application of a homogeneous model (homogeneous cylinder) gives different "effective radii" - smaller for mixtures contained protonated surfactants and larger for mixtures with deuterated surfactants.

An explanation for the large difference in intensities between scattering from pure CS and CS containing protonated aggregates is that the hydrogen atoms $(\mathrm{H})$ in $\mathrm{CS}$ are extensively substituted by $\mathrm{D}$ from the solvent.

The scattering at "zero" angle $(d \mathrm{E}(0) / d \mathrm{~S})$ obtained from fitting the parameters can be used to determine the volume fraction of surfactant in CS/surfactant complexes. This is possible because we also investigated mixtures of protonated and deuterated surfactants. The volume fraction $\left(\mathrm{V}_{\mathrm{F}}\right)$ of substituted surfactant is given by

$$
V_{F}=\frac{\left(\sqrt{R_{s}}-1\right)\left(\rho_{\operatorname{surfactan} t(H)}-\rho_{s}\right)}{\left(\rho_{C S}-\rho_{\operatorname{surfactan} t(H)}\right)-\sqrt{R_{s}}\left(\rho_{\operatorname{surfac} \tan t(D)}-\rho_{C S}\right)}
$$

where 


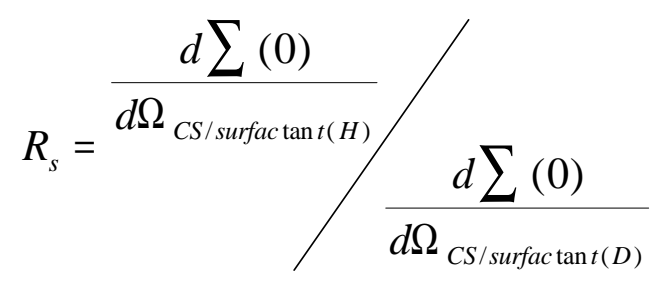

and $\rho$ denotes scattering length densities of CS, solvent $\left(\mathrm{D}_{2} \mathrm{O}\right)$ and protonated and deuterated surfactant. The volume fraction of surfactant is shown in Table VI.4. As expected, it increases with increasing surfactant concentration. The high volume fraction in $8 \mathrm{mM}$ solution supports the assumption that aggregates at this concentration have low charge so that inter-particle interactions (structure factors) can be neglected.

The single-particle scattering function of a two-shell cylinder $P^{2}(q)$ can be expressed in the same way as for a two-shell prolate ellipsoid (Eq. 48), using the form factor of a cylinder (Eq. 65). The calculated dimensions of aggregates of $\mathrm{CS}$ and NaPal are: length of aggregate $\sim 630$ $\AA$, radius of the outer cylinder (formed by CS) $\sim 35.5 \AA$ and radius of the inner cylinder (formed by NaPal) $\sim 3.3 \AA$. While the values of the dimensions and aggregation numbers are relatively uncertain, there is no doubt that the results indicate that $\mathrm{NaPal}$, in contrast to many other complexes of oppositely charged polymer-surfactant complexes, does not form spherical or ellipsoidal micellar aggregates when associating with CS. In Table VI.5 are shown some properties of other CS/surfactant complexes analysed by using this shell-core model. When the hydrocarbon chain length of the surfactant decreases (from $\mathrm{C}_{16}$ to $\mathrm{C}_{12}$ ) the radius of the shell as well as the radius of the core decreases. The values of the structure parameters of complexes of mixed $\mathrm{CS} / \mathrm{NaPal} / \mathrm{NaDod}$ aggregates are between the values of parameters of $\mathrm{CS} / \mathrm{NaPal}$ and $\mathrm{CS} / \mathrm{NaDod}$ aggregates.

In conclusion, the structure of the complexes can be roughly described as a core/shell cylinder. The shell is formed by the CS chains, which apparently, in the same way as the amylose and also, partly amylopectin, has helical conformation. The core consists of surfactant chains. Thus, the structure of CS/surfactant complexes is similar to that of inclusion complexes of amylose and surfactants, but, apparently, the cooperativity is much stronger due to the electrostatic attraction between the head groups of the surfactant and the cationic groups in CS thus leading 
to aggregates being formed at very low concentrations, with a relatively well-defined critical association concentration.

\subsubsection{Charge-neutralized CS-surfactant complexes}

The main driving forces behind polymer/surfactant association are the electrostatic attraction between the polymer substituents and the surfactant head groups as well as the hydrophobic, co-operative interaction between hydrocarbon chains of the surfactant. Hydrophobic surfactant/polymer interactions are not important. Hydrophobic interactions between the hydrophobic chains become very effective when the charge density of the polyelectrolyte is high, because the concentration of the oppositely charged surfactant in the vicinity of the polymer chain is high. This means in practice that the surfactant monomers form aggregates on the polymer at much lower concentration than in pure solution. The importance of different parameters affecting CS-surfactant interactions, is discussed in detail in articles I - IV.

The results of SAXS studies (V) show that the highly cooperative nature of polyelectrolyte/surfactant interactions not only leads association between micelles and polymers but also, when the long-range electrostatic repulsion between the surfactant/polymer aggregates is reduced by charge neutralization, to the formation of highly-ordered liquid crystalline structures. The structure of these mesophases depends on the strength of the polymer-surfactant interaction, because it depends on the complexes are formed.

\subsubsection{Structure of pure surfactant mesophases and CS/surfactant complexes}

Pure NaPal forms a crystalline hydrate in equilibrium with dilute aqueous solution at temperatures up to $\sim 60 \mathrm{EC}$ (Madelmont and Perron, 1976). The Krafft temperature of NaPal is about $40 \mathrm{E} \mathrm{C}$. At temperatures above $60 \mathrm{E} \mathrm{C}$, a hexagonal phase is formed in the concentration range $30-50 \mathrm{w} \%$. At the lower phase boundary the hexagonal phase is in equilibrium with concentrated micellar solution $(\sim 27 \mathrm{w} \%$ surfactant $)$.

Table V. 4 shows the composition of polymer/surfactant complexes in equilibrium with aqueous solutions at $60 \mathrm{E} \mathrm{C}$. First, it should be noted that this is actually a four component system which includes: CS, surfactant, water and also low molecular weight salt, $\mathrm{NaCl}$, which 
is formed in the neutralization process when the complexation between CS and oppositely charged surfactant occurs. The amount of salt is directly dependent on the amount of associated surfactant. Figure 26 shows the phase diagram of $\mathrm{CS} / \mathrm{NaPal} /$ water system. The complex phase consists of approximately $18-21 \mathrm{w} \% \mathrm{CS}, 54-62 \mathrm{w} \%$ water and $20-26 \mathrm{w} \%$ surfactant. The aqueous solution contains $0.006 \mathrm{w} \% \mathrm{CS}, 0.001 \mathrm{w} \% \mathrm{NaPal}$ and $0.05 \mathrm{~mol} \mathrm{\textrm {dm } ^ { - 3 }}$ $\mathrm{NaCl}$.

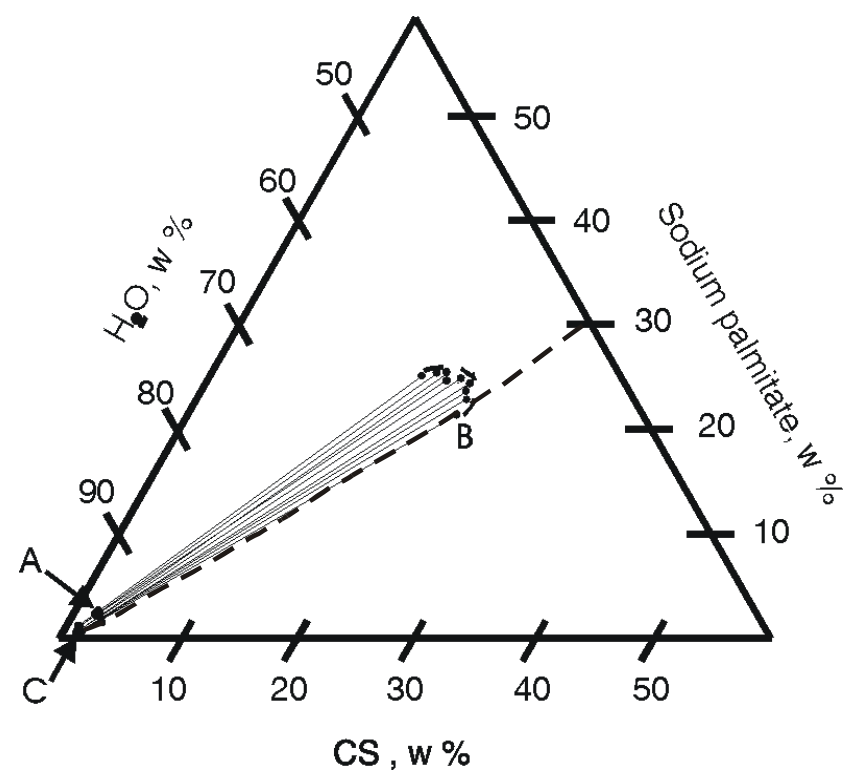

Figure 26. Partial phase diagram of the system of CS $(D S=0.80) / \mathrm{NaPal} /$ water at $60{ }^{\circ} \mathrm{C}$. Points $A$ indicate the compositions prepared in experiments; points $B$ gives analysed compositions of the complex phase and points $C$ represent the compositions of the supernatant aqueous phase. The dashed line indicates the theoretical charge neutralization. 


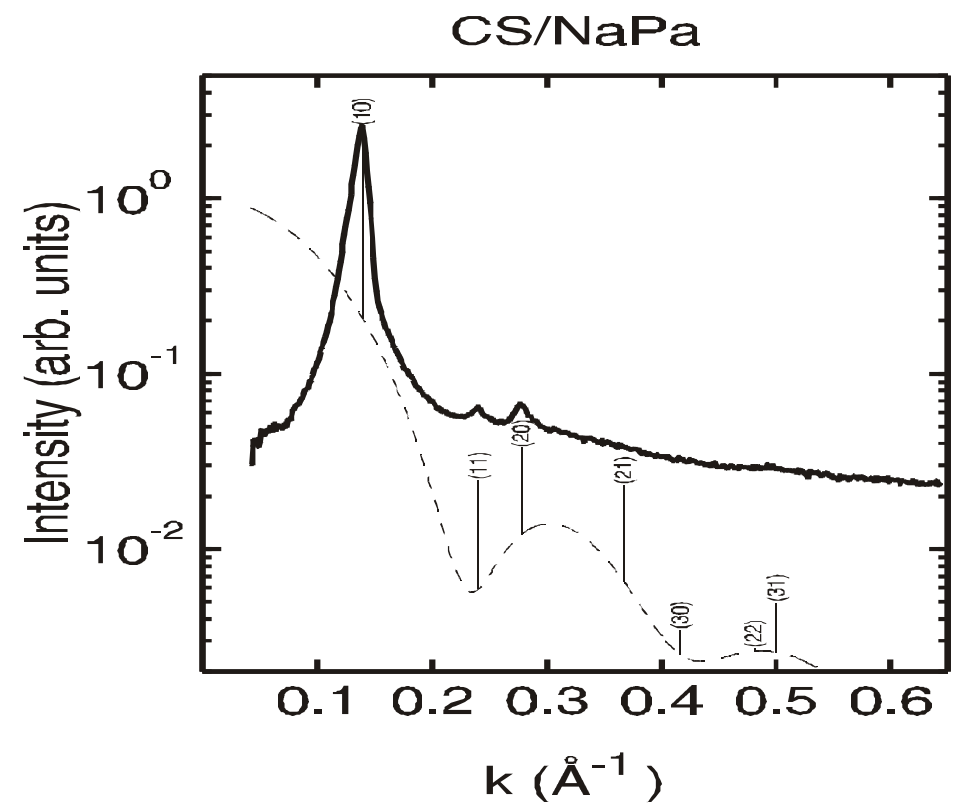

Figure 27. The SAXS intensity of the neutral CS/Pal complex at $60 \mathrm{E} C$. The vertical lines show expected reflections similarly to figure $V$. 2. The DS of starch was 0.80. The water content of complex phase was 54 w\% at $60 \mathrm{E} C$.

Figure V.3 shows the SAXS intensity curve for the CS/Pal complex at room temperature, which is well below the Krafft point of NaPal. Three positions of reflections match well with $\mathrm{NaPal} /$ water hydrate, which has a layered structure. The other peaks suggest that the complex consists of two different types of liquid crystalline phases: lamellar and $p m 3 n$ cubic with short rod-like micelles. (Fontell et al., 1985) have found the same cubic structure with pure NaPal.

When the complex is measured at 60E C, the SAXS measurements show (Fig.27) that the structure of CS/Pal complex phase is 2-d hexagonal. Thus, complexation of NaPal with CS shifts the range of existence of the NaPal hexagonal phase towards lower temperatures than for the pure surfactant. It also separates at much lower surfactant concentrations (well below the cmc of the surfactant).

Figure V.10 shows the phase diagram of $\mathrm{CS} / \mathrm{NaOl} /$ water system. The surfactant concentration in the complex phase varies from 20 to $26 \mathrm{w} \%$. In this concentration range, pure $\mathrm{NaOl}$ forms only micellar solutions. A hexagonal phase is formed in concentration range from 30 to 40 $\mathrm{w} \%$ and is stable from room temperature to 100E C (Vold 1939). The SAXS results show (Figure V.2.) that same hexagonal structure is formed by the $\mathrm{CS} / \mathrm{Ol}$ complex in the temperature range from 25 to $80 \mathrm{E} \mathrm{C}$, again in equilibrium with very dilute solution of the surfactant. 
Figure V.11 shows the phase diagram of $\mathrm{CS} / \mathrm{NaDod} /$ water system. The surfactant concentration varies from 15 to $20 \mathrm{w} \%$. In this concentration range pure sodium dodecanoate forms micelles and a cubic liquid crystalline phase at temperatures below 30E C. At higher temperatures only micellar solutions are formed. In the concentration range 30-50 w\%, NaDod forms a cubic phase below 40E C and hexagonal phase above 40E C (Madelmont and Perron, 1976). The phase behavior of CS/Dod complexes is quite similar, but the mesophases are stable at significantly lower surfactant concentrations and different temperatures. Because of the shorter chain length of NaDod, the CS/Dod complex forms $h c p$ phase in equilibrium with aqueous solution, while CS/Ol and CS/Pal form 2-d hexagonal phase (Table V.2).

The concentration of decanoate in the CS/De complex phase, separating from aqueous solution, is quite low $(<15 \mathrm{w} \%)$. No long-range ordering can be detected in this phase. The pure surfactant liquid crystalline phases are formed only at very high surfactant concentrations.

The phase behavior of $\mathrm{NaEr}$ is similar to the behavior of NaPal. The Krafft points of both surfactants are well above room temperature. $\mathrm{NaEr}$ differs from $\mathrm{NaPal}$ in that the lamellar liquid crystalline phases separate from micellar solution at quite low surfactant concentrations. The structure of CS/Er complex phase is the same as for pure NaEr, but it is formed at much lower surfactant concentrations.

The hydrocarbon chain length of SDS is quite short, but the ionic sulphate group interacts strongly with the trimethylammonium groups in the CS. This strong interaction is reflected in the composition of the complex phase. It contains from 25 to $30 \mathrm{w} \%$ of surfactant, which is much higher than in the CS/Dod complexes, although the hydrocarbon chain lengths differ by only one carbon atom. The phase in equilibrium with aqueous solution is 2-d hexagonal rather than hcp (Table V. 2), which implies that head group repulsion is more effectively screened than for CS/Dod. Pure SDS forms micellar solutions in the concentration and temperature range where the complex forms hexagonal phase (Kekicheff et al., 1989 a,b). Above 25E C, SDS forms hexagonal mesophase when the surfactant concentration exceeds $38 \mathrm{w} \%$ and the equilibrium hexagonal phase contains $40 \mathrm{w} \%$ of surfactant. In a narrow temperature range (20 $-25 \mathrm{EC}$ ) the micellar solution is in equilibrium with a crystalline hydrate of SDS. 
The charge density of CS is together with the chain length of the surfactant the other important parameter affecting to the CS/surfactant interactions and hence, the structure of the neutral complexes. The effect of charge density can be seen in that when the charge density of the polyelectrolyte decreases the complex phase loses its order and behaves like a very concentrated micellar solution. As shown by table V.3, the lowering of the charge density by a relatively small amount has a surprisingly strong effect on the structure of CS/surfactant complexes. The effect of decreasing charge density is also to increase the lattice constant of the complexes. This indicates weakened interactions between the CS/surfactant aggregates.

The effect of temperature. The temperature has two effects on the structure of CS-surfactant complexes.

1) The Krafft points of long-chain surfactants $\mathrm{NaEr}$ and $\mathrm{NaPal}$ is well above room temperature. For this reason the complexes were prepared at $60 \mathrm{E} \mathrm{C}$. Thus, when the complexes were moved to room temperature the surfactant that was not associated to CS, obviously precipitated. This precipitated surfactant hydrate is well seen in the SAXS measured at room temperature. At higher temperatures these hydrate peaks disappear. The simultaneous existence of surfactant hydrates with other liquid crystalline phases is a quite common feature of surfactant solutions at low temperatures.

2) Effect on the structure of the complexes. It is well known that the stability of lyotropic liquid crystals formed by pure surfactants decreases when the temperature rises. The free volume of the hydrocarbon chains increases and hence, the repulsion between the chains increases decreasing the CS-surfactant interaction when temperature is rising. As these results in table V. 2 show, when temperature rises the long-range ordering of the structures is weakened.

In summary, it can be concluded that crystalline phases are formed in the same sequence and with similar structures in the polyelectrolyte/surfactant system as in pure binary surfactant/water systems. However, the concentration of surfactant in the polyelectrolytecontaining phases is substantially lower than in pure surfactant system, and they also precipitate from solutions containing much less surfactant than in the binary system, i.e. the two-phase regions between liquid crystalline phase and solution are very wide. This shows that 
the effect of adding polyelectrolyte is not only an increase in the ionic strength but probably also direct binding of the polyelectrolyte to the aggregate surfaces, which reduces the electrostatic repulsion between the aggregates. This notion is further substantiated by the discussed below.

\subsubsection{Lattice constants and interparticle distances}

Table V.2 shows the lattice constants of different complex phases. These can be used as a measure of interaggregate distances. CS and sodium erucate form a lamellar mesophase with the lattice constant $54.0 \AA$ The extended chain length of erucate is $28 \AA$ This indicates that the lamellar structures have to be very close to each other because there has to be room for the CS-chains between the separate lamellas. The SANS measurements at dilute solutions (VI) show, that the surfactant molecules in complexes are incorporated into CS helix. Thus, the formation of lamellae presumably must imply some unfolding of this helix.

The extended chain length of oleate is about $23 \AA$ This value is very close the value of half of the Bragg distance (47 $\AA$ ) of hexag onal phase of CS/Ol complex (Table V.2.). In hexagonal geometry the Bragg distance is actually the distance between lattice planes. Thus, the lattice constant and interparticle distance is in this case $54.0 \AA$ It seems that the surfactant cylinders in this structure are quite close to each other, which is possible only if the CS chains are wrapped around these cylinders.

The lattice constant in the hexagonal (hcp) Dod/CS complex phase, which is also the interparticle distance, is $\mathrm{a}=53 \AA$ and $\mathrm{c}=87 \AA$ (Ta ble V.2.). The length of the surfactant monomer is in this case $15.4 \AA$ This indicates that there is more room between surfactant aggregates in this structure. This is also seen as higher water content of the complex phase (table V. 4).

The CS/Pal complex phase consists of hexagonally ordered cylinders (Table V.2.). The lattice constant at room temperature, when the structure is cubic (pm3n) is $118 \AA$ At higher temperature the structure changes to 2-d hexagonal the lattice constant and hence, the distance between the cylindrical aggregates is $55.0 \AA-52.6 \AA$ The extended chain length of Pal is 20.5 
$\AA$ Comparison of the interparticle distances of CS/O1 $(54.1 \AA+40 \mathrm{C})$ and CS/Pal $(55.0 \AA$ $+40 \mathrm{C}$ ) shows that in spite of the different chain lengths they are quite similar.

The SANS results (VI) show that the radius of CS helices is about $30 \AA$ at $70 \mathrm{C}$. Adding of $8 \mathrm{mM} \mathrm{NaPal}$ decreases the radius to about $25 \AA$ Thus, it is quite obvious that CS chains take cylindrical conformation also at high concentrations with $\mathrm{NaPal}$, and also with $\mathrm{NaOl}$. An argument in favour of this assumption is that the surfactant chain length has only a minor effect on the dimensions of cylindrical CS/surfactant aggregates. The difference in interparticle distances is also partly explained by the different water content of the two systems.

The structure of the CS/De complex phase appears to be akin to a concentrated micellar solution (Table V. 2.). The distance between the micelles is $36.0 \AA$ At room temperature. The extended chain length of De is 13 Åso there is plenty of water between the aggregates in these complexes, as is also indicated by their analytical composition (Table V. 4).

SDS and CS form very highly viscous complexes with low water content. Depending on the temperature, the distances between the cylinders vary from 42.9 to $46.0 \AA$ The dimensions of a CS/DS aggregate phase are significantly shorter, resulting in a stiffer structure and significantly lower water content of the complex phase (Table V.4).

\subsubsection{The geometry of polyelectrolyte oppositely charged surfactant aggregate phases}

Energetics of amphiphilic monolayers. The structure of surfactant aggregates depends on the bending energy of the surfactant layers in the aggregates. This bending energy leads to the formation of surfactant aggregates with different radii of curvature i.e. different geometry. The bending energy, $g_{c}$, of the layers in an aggregate can be expressed in terms the deviations from the monolayer's preferred spontaneous curvature, $c_{0}$, i.e. $\Delta \mathrm{c}_{1}=c_{1}-c_{0}$ and $\Delta c_{2}=c_{2}-c_{0}$. Thus, the bending energy is given by a two-dimensional version of Hooke's law:

$$
g_{c}=\frac{1}{2} k_{c} c_{1}^{2}+\frac{1}{2} k_{c} c_{2}^{2}+\bar{k} \Delta c_{1} \Delta c_{2}
$$


where $k_{c}$ is the bending modulus and $\bar{k}$ is a coupling constant. The bending energy depends on the intermolecular forces between the amphiphile in the monolayers, including structural forces, hydrogen bonding, electrostatic repulsion, steric repulsion, van der Waals' attractions, hydrophobic interactions and solvation forces.

The structural force, due to the collisions between the molten hydrocarbon chains creates an outward pressure, tending to expand the hydrocarbon moiety. This is clearly reflected in the temperature dependence of the curvature of the aggregate structures both in pure surfactant/ water and CS/surfactant/water systems.

At the polar/non-polar interface, hydrophobic interactions create an inward pressure that tends to reduce water-oil contact. In the head group region there are steric, electrostatic and hydration forces, which are expected to create a net positive outward pressure. When the outward pressure in the chain region is high the monolayer is likely to bend towards water. On basis of presently available results, the importance of hydrophobic, steric and hydration forces in the CS/surfactant/water systems are difficult to assess. However, the strong interaction between sulphate and trimethylammonium groups in the CS/DS complexes leads to formation of aggregates with lower curvature than in corresponding CS/Dod systems, which indicates that reduction of hydrophobic interactions and solvation in the surface plays a role.

The electrostatic contributions to the bending modulus of mean curvature, $k_{c}$. It can be deduced from the results described above is that phases similar to those formed by the pure surfactants are formed by the CS/surfactant complexes, but at considerably lower surfactant concentrations. It seems reasonable to assume that the major reason for this effect is the electrostatic interaction between the polyelectrolyte and the surfactant aggregates. Considering lamellar structures, there are three characteristic length scales: the mean membrane separation $2 d$, the Debye-Hückel screening length $\kappa^{-1}$ and the Gouy-Chapman length $\lambda=e / 2 \pi l \sigma$, where $\sigma$ is the surface charge density, $e$ is the electronic unit charge, $l=e^{2} / 4 \pi \varepsilon T$ is the Bjerrum length, and $\varepsilon$ is the dielectric constant of the solvent. In the limit of high electrolyte concentration ( $\kappa d>1$ and $\kappa \lambda<1$ ), the solutions of linearised Poisson-Bolzmann equation for several geometries all indicate that the electrostatic contribution to the bending constant $k_{\mathrm{c}}$ is given by (Harden et al., 1992): 


$$
k_{c}=\frac{3 T}{4 \pi \kappa^{3} l \lambda^{2}}
$$

where

$$
\kappa=\left(\frac{e^{2} \sum n_{i}^{0} z_{i}^{2}}{\varepsilon \varepsilon_{0} k T}\right)^{1 / 2}
$$

and $n_{i}^{0}$ and $z_{i}$ are the number concentration and valency, respectively, of ion $i$. Because $6^{-1}$ increases with increasing electrolyte concentration, the bending constant between charged particles $k_{c}$ decreases very rapidly when the electrolyte concentration increases. For example, when the electrolyte concentration (1-1 electrolyte) increases from $1 \mathrm{mM}$ to $10 \mathrm{mM}$, $\kappa^{-1}$ decreases from $9.6 \mathrm{~nm}$ to $3.04 \mathrm{~nm}$, and the bending constant is reduced to about $6 \%$ of its initial value.

The screening of electrostatic repulsion does, indeed, explain the reduction in the bending energy caused by ionic interactions between the polar head groups. This is manifested in the gradual transitions from micellar to saddle to lamellar phases as the concentration of surfactant in binary water/surfactant systems increases. The same effects can be obtained by adding a simple electrolyte. However, it is evident that the oppositely charged polyelectrolyte neutralizes the charges much more effectively than a simple electrolyte. Adsorption of polyelectrolyte to the monolayer (or micellar) surface neutralizes the charges by direct ion binding. This markedly reduces the electrostatic contribution to the bending energy (Eq. 69) and, hence the surface curvature of surfactant aggregates is changed, leading to stabilization of phases with lower curvature than micelles. As Equation (70) shows, the bending modulus depends strongly on the Gouy-Chapman lengths and, hence, on the charge density i.e. the packing of the polar head groups of the surfactant monomers. The CS has very high charge density; therefore the association of surfactant aggregates with CS chains with high charge, via charge neutralization, results in a very effective shortening of $\lambda$ and, hence, also a lower bending energy and a closer packing of surfactant monomers in aggregates of low curvature. Similar conclusions were reached in a recent study of sodium polyacrylate/ cetyltrimethylammonium bromide systems (Ilekti et al., 1999). 
When the ionic strength increases, the phase boundaries of lyotropic liquid crystalline phases move towards lower surfactant concentrations. They also shift to higher temperatures, indicating increased stability of the surfactant aggregates. However, this effect is much weaker than the effect of adding polyelectrolyte. For instance, the phase diagram of the $\mathrm{NaPal} / \mathrm{NaCl} /$ water system (Laughlin 1994) shows that addition of up to $5 \mathrm{w} \%$ of $\mathrm{NaCl}$ to a $1 \mathrm{w} \% \mathrm{NaPal}$ solution does not cause any phase changes. In our investigation, the initial $\mathrm{NaPal}$ concentration of the CS/Pal sample was $1 \mathrm{w} \%$ and the CS concentration $1.2 \mathrm{w} \%$. The estimated effect of CS on the ionic strength corresponds approximately to $0.1 \mathrm{w} \%$ of added $\mathrm{NaCl}$. This indicates very clearly that the addition of polyelectrolyte has much stronger effect than the addition of a simple electrolyte.

Because the formation of more or less neutral complexes when the polycation associates with anionic surfactant, there is also an ionic strength effect due to the release of simple ions from the polyelectrolyte and the micelles. This could explain part of the detected shift of the phase boundaries and areas of liquid crystalline phases of CS/surfactant complexes, but it is clear from the discussion above that this effect is minor.

\subsubsection{Rheology of the complexes}

\subsubsection{General}

The rheological behaviour of highly concentrated CS solution is shown in Figures III.2a. and III.2b. Complex modulus is nearly identical to the loss modulus. Thus, viscosity is the predominating rheological property. This behaviour is characteristic of many polymer solutions. Addition of an anionic surfactant so that complex phase is formed, affects the rheological properties very significantly. A typical pattern of behaviour is exemplified by the CS/SDS system in Figure 28. The dynamic modulus is two orders of magnitude larger than that of surfactant-free starch. The interaction between CS and the surfactant results in a strongly increased elastic modulus of the complex phase. The complexes are also slightly thixotropic (Fig. III.3). 


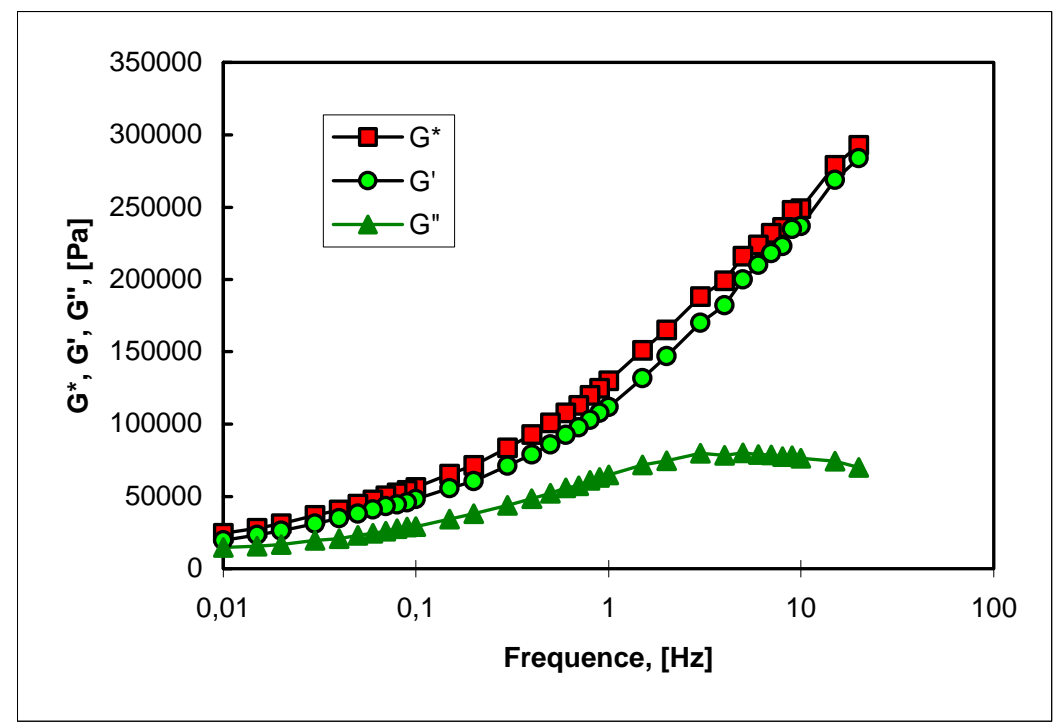

Figure 28. Dynamic rheological behaviour of CS/DS complex phase. Degree of substitution of starch was 0.772. $T=298 \mathrm{~K}$.

Charge density of the polymer. In Table III. 2 it can be seen that the yield stress increases when the DS of the CS increases. The existence of yield stress at higher shear rates indicates that the structure of the complex is somewhat stronger. When the DS of the CS increases, both viscosity and elasticity of the complex phase increase, but it is the increase in the elasticity that dominates. Thus, the CS/surfactant interaction in the complex phase is stronger (I). This can be seen also in the fact that the maximum swelling of neutral complex phases with water decreases with increasing charge density of the cationic starch. The other possibility is that the association of surfactants with CS of higher DS forms complexes with lower water content and also different liquid crystalline phases of the surfactants.

Surfactant chain length. As the hydrocarbon chain length of the surfactant increases, the viscosity and the dynamic module of the complex phase rapidly increase. On the other hand, it is known that the long-chain surfactants associate with the polymers in lower concentrations than the short-chain surfactants do. The growth of length of the hydrocarbon chain strengthens the interaction between the CS and surfactant (I, II). In addition, the changes of surfactant liquid crystal phases $(\mathrm{V})$ probably contribute to the rheological properties of the complex phase, but our data do not allow any conclusions regarding these effects.

Electrolyte concentration. The electrostatic interactions are increasingly screened when the electrolyte concentration increases. These weakened interactions show up as decreasing yield stress, decreasing viscosity and increasing water content of the complex phase in equilibrium 
with aqueous solution. The structure of the complex phase comes more swollen because the interaction between polyelectrolyte and oppositely charged ionic end-groups of the surfactant is weakened. In spite of the decreasing elasticity and viscosity of the complex the elasticity/viscosity ratio is not affected by the increasing electrolyte concentration.

The effect of surfactant mixture. It is well known that mixing of two surfactants with the same functionality produces mixed micelles that include both individual surfactant monomers. It has also been shown that these mixed micelles primary consist of the long-chain surfactant and the fraction of the short-chain surfactant is very much lower (IV), (Shinoda, 1954), in the micelles than in the surrounding solution.

\subsubsection{Fitting of the results to simple rheological models}

Many different phenomenological equations have been used to describe the viscoelastic behaviour of different systems. At best, the theoretical background of these is limited. However, they are quite useful for the phenomenological comparison of the viscoelastic properties of different systems. Hence, the experimental data is fitted to the commonly used equations by a simple least squares procedure. Used models were:

Power law

$$
\sigma=k \gamma^{n}
$$

Bingham plastic

$$
\sigma=\sigma_{y}+k \dot{\gamma}
$$

Herschey-Bulkley

$$
\sigma=\sigma_{y}+k \gamma^{n}
$$

Casson

$$
\sigma=\sigma_{y}^{1 / 2}+k \gamma^{i / 2}
$$

Vocaldo

$$
\sigma=\left(\sigma_{y}^{1 / n}+k \gamma\right)^{n}
$$

where $\mathrm{F}$ is the shear stress, ( is the shear rate, $\mathrm{F}_{y}$ is the yield stress and $k$ is a measure of the viscosity at high shear rates.

Figure 29 shows the correlation of these models with the rheological data of several samples. Clearly, the Bingham plastic or power law models do not fit the experimental data. Using the more complex Casson or Vocaldo models provides no improvement over the Herschel-Bulkley (HB) model, which, moreover, correlates very well indeed with the results. Therefore, the 
following discussion is based on the parameters obtained by fitting the HB model to the data. Table III 3, summarises the fitting of the HB model. The yield stress $F_{y}$ increases strongly with increasing chain length of the surfactant and with increasing polyelectrolyte charge density. It is very much higher for SDS than for KDod or NaOl. The yield stress $\mathrm{F}_{\mathrm{y}}$ also decreases when the electrolyte concentration increases.

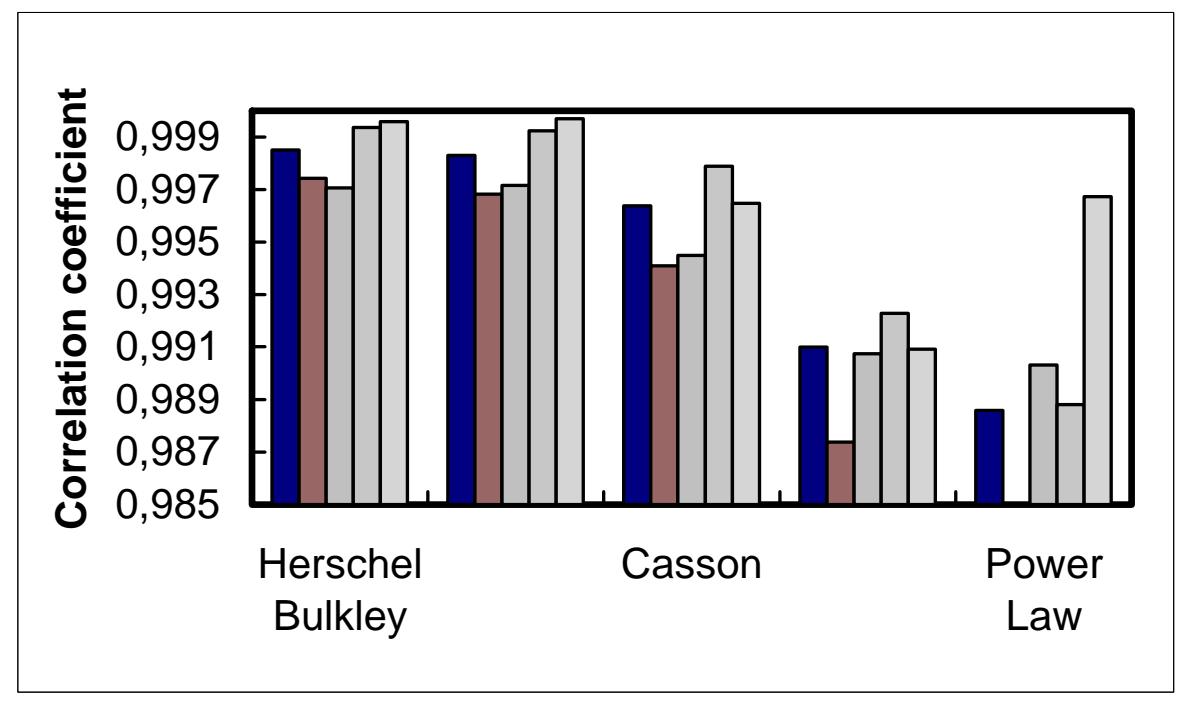

Figure 29. Correlation coefficients of different rheological models with stress/strain relationship of different surfactant complex phases. The baulks represent different $C S /$ surfactant complexes. From left to right: $C S(D S=0.40)+K D o d, C S(D S=0.40)+\mathrm{NaOl}$, $C S(D S=0.40)+S D S, C S(D S=0.78)+K D o d$ and $C S(D S=0.78)+N a O l$.

The value of the parameter $k$ that represents the slope of the shear rate/viscosity curve increases rapidly when the charge density of the polymer or the chain length of the surfactant increases. This means that the viscosity and elasticity of the system is increases when the DS of the starch or the chain length of the surfactant is increases, as also shown by the dynamic experiments. When the ionic strength of the system increases the viscosity decreases. On the other hand, the exponent $n$ is almost independent of surfactant chain length, end group or ionic strength. The value of $n$ varies from 0.64 to 0.76 . Thus, the value of $n$ that is clearly below 1 , as expected for a pseudoplastic gel. 


\subsubsection{Rheology vs. structure of CS/surfactant complexes}

Dilute aqueous solutions. It is evident from the cooperative nature of the interaction (I) and from the special character of CS conformation (VI) that the surfactant associated with cationic starch forms inclusion-like complexes. It is possible that in the complexes the aggregates interact with other starch molecules, creating a loose network structure. It is plausible to assume that because of their dynamic structure, the aggregates do not form solid, linked network structures. Instead, they form a weak, very elastic and renewable structure.

Concentrated complex phase. Recent studies discussing the structure of polyelectrolyte/ oppositely charged surfactant complexes (Ponomarenko et al., 1998 and Ilekti et al., 1999) have shown that the structure of these kind of concentrated complexes is quite different from what has been believed previously. The complexes consist of highly ordered liquid crystal phases of surfactants where the polymer is anchored. The surfactants can form, for example lamellar, hexagonal or cubic phases in polyelectrolyte/surfactant complexes. As it has been shown (VI) CS/surfactant complexes have the same kind of structure. The results indicate (IV) that the interaction between this type of mixed micelles and CS depends on the chain length difference of these surfactants and also on the molar ratio of these surfactants. The results showed that the interaction between CS and surfactants is the weaker the higher is the fraction of the short-chain surfactant and the bigger is the chain length difference of the surfactants.

The effect of the second surfactant on the rheology of these CS/surfactant complex phases is well explained by the effect of the second surfactant on the CS/surfactant interaction. The effect is quite similar to the effect of using the shorter-chain surfactant alone. Thus, when the fraction of the short-chain component increases one approaches the situation obtaining for pure CS/shorter-chain surfactant complexes. This can also be observed as increasing water content of the complex phase in equilibrium with aqueous solution (IV). 


\section{CONCLUSIONS}

CS/anionic surfactant interactions can be understood by assuming that the most important factors governing the behaviour on the systems are cooperative hydrophobic interactions between surfactant chains and electrostatic interactions between cationic and anionic polyelectrolytes Hydrophobic polymer/surfactant interactions are of minor importance. The enhanced surface activity of these systems at very low surfactant concentrations is due to formation of surface-active complexes by way of counter ion condensation. Critical association concentrations are observed at concentrations well below the $c m c$ s of the surfactants.

There is a minimum hydrocarbon chain length of the surfactant and also a minimum degree of substitution of the CS to association between the CS and surfactant to take place.

Associative phase separation occurs in extremely dilute systems when the charge ratio between the surfactants and the polymers is close to one.

The effect of mixing on the micellisation of the binary surfactant solutions can be described to a good approximation by taking into account only the effects of the volume difference between the hydrocarbon chains. Complex formation on CS depends on the chain-length difference in exactly the same way as for free mixed micelles.

The separated complex phase is a hydrophobic, highly viscous and gel-like containing 40 to 60 w\% of water. The high viscosity and low water content of the complex phase indicates that the interactions between the CS and anionic surfactants are very strong. The water content of the complex phase decreases when the chain length of the surfactant or the DS of the CS increases, indicating increased polymer/surfactant interaction.

With mixed surfactants the water content of the complex phase increases when the chain length difference increases. The more surface-active component is strongly enriched in the polymer complexes.

When excess surfactant is added, the separated complex phase redissolutes completely or partly, depending on the charge density of the CS. The redissolution of the complex phase takes place by the charge reversal mechanism 
SANS indicates that mixed micelles of alkanoates are prolate ellipsoids with the shorter chain surfactant enriched in the end-cups of the ellipsoid. SANS also indicates that in dilute solutions CS molecules are roughly cylindrical i.e. have helical conformation. When they associate with surfactants, aggregates are formed, in which a core of surfactant is surrounded by CS. The surfactant monomers do not form any micelle-like aggregates in these complexes. The structure of the complexes resembles the inclusion complexes of amylose and surfactants. In mixed surfactant systems, the longer chain surfactant is enriched into the surfactant/CS complex.

As the SAXS results show, the charge equivalence CS/surfactant complexes consist of a lyotropic liquid crystalline phase. Depending on the chain length of the surfactant, temperature and salt concentration, the structure of the liquid crystalline phase is hexagonal, lamellar or cubic. The mesophases are the same as the pure surfactants in forming without any added polymer, but they form with CS at much lower surfactant concentrations. Thus, the polymer act as a huge and very effective counter ion that screens repulsion between the surfactant head groups and the surfactant aggregates.

The gel-like CS/surfactant complex phases have high viscosity and elasticity. They show pseudoplastic behaviour. The rheological behaviour of the complexes is described by the Hershel-Bulkley model. The rheological data for the complex phases are compatible with the finding that the surfactants form liquid crystalline structures with the polymer anchored to the surfactant aggregates. 


\section{REFERENCES}

Abrahmsen-Alami, S. and Stilbs, P. (1994), ${ }^{1} \mathrm{H}$ NMR self-diffusion and multifield ${ }^{2} \mathrm{H}$ spin relaxation study of model associative polymer and sodium dodecyl sulfate aggregation in aqueous solution, J. Phys. Chem. 98, 6359 - 6367.

Abuin, E. B. and Scaiano, J. C. (1984), Exploratory study of the effect of polyelectrolytesurfactant aggregates on photochemical behavior, J. Am. Chem. Soc. 106, 6274 - 6283.

Adam, N. K. (1968), The Physics and Chemistry of Surfaces, Dover, New York, 2nd ed., 1968.

Adamson, A. W. (1984), Physical Chemistry of Surfaces, Interscience Publishers, New York, 3rd ed., 1984.

Almgren, M., Hansson, P., Mukhtar, E. and van Stam, J. (1992). Aggregation of alkyltrimethylammonium surfactants in poly(styrene sulfonate) solutions, Langmuir 23, 2405 2412.

Ananthapadmanabban, K. P., Leung, P. S. and Goddard, E. D. (1985), Fluorescence and solubilization studies of polymer-surfactant systems, Colloids Surf. 13, 63 - 72.

Anderson, C. F. and Record, M. T. (1982), Polyelectrolyte theories and their applications to DNA, Annu. Rev. Phys. Chem. 33, 191 - 222.

Anthony, O. and Zana. R. (1996), Interactions between water soluble polymers and surfactants: effect of the polymer hydrophobicity. 1. Hydrophilic polyelectrolytes, Langmuir 12, 1967 - 1975.

Antonietti, M., Conrad, J. and Thunemann, A. (1994), Polyelectrolyte_surfactant complexes: a new type of solid, mesomorphous material, Macromolecules 27, 6007 - 6011.

Antonietti, M., Kaul, A. and Thunemann, A. (1995), Complexation of lecithin with cationic polyelectrolytes. 'Plastic membranes' as models for the structure of the cell membrane? Langmuir 11, 2633 - 2638.

Antonietti, M. and Wenzel, A. (1998), Structure control of polyelectrolyte-lipid complexes by variation of charge density and addition of cholesterol. Colloids and Surfaces A 135, 141 147. 
Bakeev, K.N., Chugunov, S.A. , Teraoka, I., MacKnight, W.J., Zezin, A. and Kabanov, V. A., (1994), Complexation of ionomers and surfactant molecules of the same charge in a nonpolar solvent Macromolecules 27, 3926 - 3932.

Bakeev, K. N., Ponomarenko, E. A., Shishkanova, T. V., Tirrell, D. A., Zezin, A. B., Kabanov, V.A.; (1995), Polyelectrolytes and oppositely charged surfactants in organic solvents: From reversed micelles to soluble polymer_surfactant complexes. Macromolecules 28, 3657 - 3663.

Barneveld, P.A., Scheutjens, J.M.H.M.; Lyklema, J. (1992), Bending moduli and spontaneous curvature. 1. Bilayers and monolayers of pure and mixed nonionic surfactants. Langmuir 8, $3122-3130$.

Barneveld, P.A., Hesselink, D.E.; Leermakers, F.A.M.; Lyklema, J.; Scheutjens, J.M.H.M. (1994). Bending moduli and spontaneous curvature. 2. Bilayers and monolayers of pure and mixed ionic surfactants. Langmuir 10, 1084 - 1092.

Bekturov, E. A., Kudaibergenov, S. E. and Kanapyanova, G. S. (1984), Interaction of synthetic polyampholytes with anionic and cationic detergents in aqueous solution, Polym. Bull. 11, 551 $-555$.

Berr, S. S., Coleman, M. J., Jones, R. R. M., Johnson, J. S. (1986) Small angle neutron scattering study of the structural effects of substitution of tetramethylammonium for sodium as the counterion in dodecyl sulfate micelles. J. Phys. Chem. 90, 6492 - 6499.

Bevington, B. R. (1969), Data Reduction and Error Analysis for Physical Sciences; McGrawHill: New York, 1969.

Bradstreet, R. B. (1965), The Kjeldahl Method for Organic Nitrogen, Academic Press, New York, 1965.

Brumberger, H. (Ed.), Modern Aspects of Small-Angle Scattering. Kluwer Academic Publishers, The Netherlands, 1995.

Cabane, B., Duplessix, R.(1982), Organization of surfactant micelles adsorbed on a polymer molecule in water: a neutron scattering study. J. Physique 43, 1529 - 1542.

Carlsson, A., Karlström, G. and Lindman, B. (1989), Characterization of the interaction between a nonionic polymer and a cationic surfactant by the Fourier transform NMR selfdiffusion technique, J. Phys. Chem. 93, 3673 - 3677. 
Carnali, J. O. (1993), (Polymer/polymer)-like phase behavior in systems tetradecyltrimethylammonium bromide/sodium polyacrylate/water, Langmuir 9, 2933 - 2941.

Chen, L., Yu, S., Kagami, Y., Gong, J., Osada, Y.(1998), Surfactant binding of polycations carrying charges on the chain backbone: cooperativity, stoichiometry and crystallinity. Macromolecules 31, 787 - 794.

Chen, S. H. (1986) Small angle neutron scattering studies of the structure and interaction in micellar and microemulsion systems. Ann. Rev. Phys. Chem. 37, 351 - 399.

Chevalier; Y., Zemb, T. (1990) The structure of micelles and microemulsions. Rep. Prog. Phys. 53, 279 - 371.

Chu, D. and Thomas, J. K. (1986), Effect of cationic surfactants on the conformational transition of poly(methacrylic acid), J. Am. Chem. Soc. 108, 6270 - 6276.

Codet, M. C., Buleon, A., Tran, V. and Colonna, P. (1993) Structural features of fatty acidamylose complexes, Carbohydrate Polymers 21, 91 - 95.

Corrin, M. L. and Harkins, W. D. (1947), The effect of salts on the critical concentration for the formation of micelles in colloidal electrolytes, J. Am. Chem. Soc. 69, 683 - 688.

Cotton, J. P. (1991), In Neutron, X-Ray and Light Scattering: Introduction to an Investigative Tool for Colloidal and Polymeric Systems; Lindner, P. and Zemb, T., Eds; North-Holland: Amsterdam, 1991.

de Gennes, P. G. (1990), Interaction between polymers and surfactants, J. Phys. Chem. 94, 8407 - 8413.

Dubin, P. L., Rigsbee, D. R. , Gan, L.-M. and Fallon, M. A. (1988), Equilibrium binding of mixed micelles to oppositely charged polyelectrolytes, Macromolecules 21, 2555 - 2559.

Dubin, P. L., The, S. S., Gan, L.-M. and Chew, C. H.(1990), Static light scattering of polyelectrolyte-micelle complexes, Macromolecules 23, 2500 - 2506.

Effing, J. J., McLennan, I. J. and Kwak, J. C. T. (1994), Associative phase separation observed in a hydrophobically modified poly(acrylamide)/sodium dodecyl sulfate system, J. Phys. Chem. 98, 2499 - 2502. 
Einstein, A. (1956), Investigation on the Theory of the Brownian Movement, Dovel Publications, New York, 1956, 58 pp.

Eliasson, A. C. and Kim, H. R. (1995), Dynamic rheological method to study the interaction between starch and lipids. J. Rheol. 39, 1519 - 1534.

Evans, D.F. and Wennerström, H. (1999), The Colloidal Domain, VCH Publishers, New York.

Feigin, L.A. and Svergun, D.I. (1987), Structure Analysis by Small-Angle X-Ray and Neutron Scattering, Plenum Press, New York, 1987.

Finch, J. A. and Smith, G. W. (1973), Dynamic surface tension of alkaline dodecylamine solutions, J. Colloid Interface Sci. 45, 81 - 91.

Fixman, M. (1979), The Poisson-Boltzmann equation and its application to polyelectrolytes, $J$. Chem. Phys. 70, 4995 - 5005.

Flory, J. P. (1953), Principles of Polymer Chemistry, Cornell University Press, Ithaca, New York,1953.

Fontell, K., Fox, K. K., and Hansson, E. (1985), On the structure of the cubic phase I1 in some lipid-water systems. Mol. Cryst. Liq. Cryst., Lett. Sect. 1(1-2), 9-17.

Francois, J., Dayantis, J. and Sabbadin, J. (1985), Hydrodynamical behaviour of the poly(ethylene oxide) - sodium dodecyl sulphate complex, Eur. Polym. J. 21, 165 - 174.

Fundin, J. and Brown, W. (1994), Polymer/surfactant interactions. Sodium poly(styrenesulfonate) and CTAB complex formation. Light scattering measurements in dilute aqueous solution, Macromolecules 27, 5024 - 5031.

Förster, S., Schmidt, M. and Antonietti, M. (1990), Static and dynamic light scattering by aqueous polyelectrolyte solutions: effect of molecular weight, charge density and added salt, Polymer 31, 781 - 792.

Garamus, V. M. (1997), Study of mixed micelles with varying temperature by small_angle neutron scattering. Langmuir 13, 6388 - 6392.

Glatter, O., Kratky, O. (1992). Small Angle X-ray Scattering, Academic Press, London 1992. 
Goddard, E. D. and Hannan, R. B. (1976), Cationic polymer/anionic surfactant interactions, $J$. Colloid Interface Sci. 55, 73 - 79.

Goddard, E. D. and Hannan, R. B. (1977), Polymer/surfactant interactions, J. Am. Oil Chem. Soc. 54, 561-566.

Goddard, E. D. and Leung, P. S. (1982), Complexes of cationic polymers and anionic surfactants. Polym. Prepr. Am. Chem. Soc. Div. Polym. 23, 47 - 47.

Goddard, E. D. (1986a) Polymer-surfactant interaction: Part I. Uncharged water soluble polymers and charged surfactants. Colloids Surf. 19, 255 - 300.

Goddard. E. D. (1986b) Polymer-surfactant interaction: Part II. Polymer and surfactant of opposite charge. Colloids Surf. 19, 301 - 329.

Goddard, E. D. and Ananthapadmanadhan, K. P. eds. (1993), Interactions of Surfactants with Polymers and Proteins, CRC Press, USA 1993, 427 pp.

Gorski, N., Gradzielski, M., Hoffmann, H. (1994), Mixtures of nonionic and ionic surfactants. The effect of counterion binding in mixtures of tetradecyldimethylamine oxide and tetradecyltrimethylammonium bromide. Langmuir 10, 2594 - 2603.

Hahn, T., (Ed.) International Tables for Crystallography; D. Reidel Publishing Co., Dortrecht, The Netherlands, 1985, 128pp.

Hansen, J. P., Hayter, J. B. (1982), A rescaled MSA structure factor for dilute charged colloidal dispersions. Molecular. Phys. 46, 651 - 656.

Hansson, P and Almgren, M. (1994), Interaction of alkyltrimethylammonium surfactants with polyacrylate and poly(styrenesulfonate) in aqueous solution. Phase behavior and surfactant aggregation numbers. Langmuir 10, 2115 - 2124.

Hansson, P. and Almgren, M. (1995) Large $\mathrm{C}_{12} \mathrm{TAB}$ micelles formed in complexes with polyvinyl sulfate and dextran sulfate. J. Phys. Chem. 99, 16694 - 16703.

Hansson, P. (1998), Surfactant self_assembly in polyelectrolyte gels: aggregation numbers and their relation to the gel collapse and the appearance of ordered structures in the $\mathrm{NaPa} / \mathrm{C}_{12} \mathrm{TAB}$ system. Langmuir 14, 4059 - 4064. 
Harada, A. and Nozakura, S. (1984), Formation of organized structures in systems of polyelectrolyte-ionic surfactants. Polym Bull. 11, 175 - 178.

Harden, J. L., Marques, C., Joanny, J.-F. (1992), Membrane curvature elasticity in weakly charged lamellar phases. Langmuir 8, 1170 - 1175.

Hayakawa, K. and Kwak, J. C. T. (1983 a), Study of surfactant-polyelectrolyte interactions. II. Effect of multivalent counterions on the binding of dodecyltrimethylammonium ions by sodium dextran sulfate and sodium poly(styrenesulfonate) in aqueous solution, J. Phys. Chem. 87, 506 - 509.

Hayakawa, K. , Santerre, J. P. and Kwak, J. C. T. (1983 b), Study of surfactant-polyelectrolyte interactions. Binding of dodecyl- and tetradecyltrimethylammonium bromide by some carboxylic polyelectrolytes, Macromolecules 16, 1642 - 1645.

Hayakawa, K. and Kwak, J.C.T. (1991) in Cationic Surfactants, Rubingh, D.N. and Holland, P.M. eds., Marcel Dekker, New York, 2nd ed., 1991.

Hayter, J. B. and Penfold, J., (1981) Analytical structure factor for macroion solutions. Mol. Phys., 42, 109 - 118.

Herslof, Å, Sundelof, L.-O. and Edsman, K. (1992), Interaction between polyelectrolyte and surfactant of opposite charge. Hydrodynamic effects in the sodium hyaluronate/ tetradecyltrimethylammonium bromide/sodium chloride/water system. J. Phys. Chem. 96, $2345-2348$.

Holland, P. M. and Rubingh D.N. (1992), Mixed Surfactant Systems, ACS Symposium series 501, American Chemical Society, Washington, 1992.

Huh, C. and Mason, S. G. (1975), A rigorous theory of ring tensiometry, Colloid Polymer Sci. 253, 566 - 580 .

Ilekti, P., Piculell, L., Tournilhac, F. and Cabane, B. (1998), How to concentrate an aqueous polyelectrolyte/surfactant mixture by adding water. J. Phys. Chem. B 102, 344 -351.

Ilekti,. P.; Martin, T.; Cabane, B.; Piculell, L. (1999), Effects of polyelectrolytes on the structures and interactions of surfactant aggregates. J. Phys. Chem. 103, 9831-9840. 
Israelachvili, J. N., Mitchell, D. J. and Ninham, B. W. (1976), Theory of self-assembly of hydrocarbon amphiphiles into micelles and bilayers, J. Chem. Soc. Faraday Trans. 2, 72, 1525 $-1568$.

Jones, M. N. (1967), The interaction of sodium dodecyl sulfate with polyethylene oxide , $J$. Colloids Interface Sci. 23, 36 - 42.

Jönsson, B., Lindman, B., Holmberg, K., Kronberg, B. (1998), Surfactant and Polymers in Aqueous Solution, Wiley: New York, 1998, ch. 3.

Ju, R. T. C, Frank, C. W. and Gast, A. P. (1992), Contin analysis of colloidal aggregates, Langmuir 8, 2165 - 2171.

Kamenka, N., Burgaud, I., Zana, R. and Lindman, B. (1994), Electrical conductivity, selfdiffusion and fluorescence probe investigations of the interaction between sodium dodecyl sulfate and ethyl(hydroxyethyl)cellulose, J. Phys. Chem. 98, 6785 - 6789.

Karlström, G., Carlsson, A. and Lindman, B. (1990), Phase diagrams of nonionic polymerwater systems. Experimental and theoretical studies of the effects of the surfactants and other cosolutes, J. Phys. Chem. 94, 5005 - 5015.

Kekicheff, P., Grabielle-Madelmont, C., Ollivon, M. (1989a) Phase diagram of sodium dodecyl sulfate-water system. 1. A calorimetric study. J. Colloid Interface. Sci. 131, 112 - 132.

Kekicheff, P. (1989b) Phase diagram of sodium dodecyl sulfate-water system. 2.

Complementary isoplethal and isothermal studies. J. Colloid Interface. Sci. 131, 133 - 152.

Khandurina, Y. V., Rogacheva, V. B., Zezin, A. B., Kabanov, V. A. (1994a), Interaction of net polyelectrolytes with oppositely charged surfactants. Polym. Sci. 36, 184 - 188.

Khandurina, Y. V., Dembo, A. T., Rogacheva, V. B., Zezin, A. B., Kabanov, V. A. (1994b), Structure of polycomplexes composed of cross-linked sodium polyacrylate and cationic micelle forming surfactants. Polym. Sci. 36, 189 - 194.

Khandurina, Y. V., Rogacheva, V. B., Zezin, A. B., Kabanov, V. A. (1994c), Stability of polycomplexes of network polyelectrolytes with surface-active substances in aqueous salt and aqueous organic media. Polym. Sci. 36, 195 - 199.

Kiefer, J.J., Somasundaran, $\mathrm{P}$ and Ananthapadmanablan in Polymer Solutions, Blends and Interfaces (I. Noda and D.N. Rubingh, eds.). Elsevier Science Publ., 1992, pp.423 - 444. 
Kim, R., Ishizawa, M., Gong, J., Osada, Y. (1999), Molecular and supramolecular structures of complexes formed by polyelectrolyte_surfactant interactions: effects of charge density andcompositions. J. Polym. Sci. A 37, 635 - 644.

Klevens, H. B. (1953), Structure and aggregation in dilute solutions of surface active agents, $J$. Am. Oil Chem. Soc. 30, 74 - 80.

Koene, R. S. and Mandel, M. (1983a), Scaling relations for aqueous polyelectrolyte - salt solutions. 1. Quasi-elastic light scattering as a function of polyelectrolyte concentration and molar mass, Macromolecules 16, 220 - 227.

Koene, R. S., Nicolai, T. and Mandel, M. (1983b), Scaling relations for aqueous polyelectrolyte - salt solutions. 2. Quasi-elastic light scattering as a function of polyelectrolyte concentration and salt concentration, Macromolecules 16, 227 - 231.

Koene, R. S., Nicolai, T. and Mandel, M. (1983c), Scaling relations for aqueous polyelectrolyte - salt solutions. 3. Osmotic pressure as a function of molar mass and ionic strength in semidilute regitime, Macromolecules 16, 231 - 236.

Korobko, T.A., Izumrudov, V. A., Zezin, A. B., Kabanov, V. A. (1994), The role of nonpolar interactions in the reactions of nonstoichiometric interpolyelectrolyte complexes with anionic surfactants. Polym. Sci. 36, 179 - 183.

Kosmella. S., Kötz, J., Friberg, S.E., MacKay, R. (1996) Interactions of polyelectrolytes with the lyotropic liquid crystalline system hexadecyltrimethylammonium bromide/decanol/water. Coll. Surf. A 112, 227 - 231.

Kotlarchyk, M., Chen, S. H.(1983), Analysis of small angle neutron spectra from polydisperse interacting colloids, J. Chem. Phys. 79, 2461 - 2469.

Kubik, S., Höller, O., Steinert, A., Tolksdorf, M. and Wulff, G. (1995), Inclusion compounds of derivatized amyloses. Macromol. Symp. 99, 93 - 102.

Landau, L.D. and Lifshitz, E.M. (1985). Classical Theory of Fields, Pergamon Press, Oxford, 1985.

Lange, H. (1971), Wechselwirkung zwishen natrium alkylsulfaten und polyvinylpyrrolidon in wässrigen lösungen, Kolloid Z. Z. Polym. 243, 101 - 109. 
Laughlin, R. G. (1994), The Aqueous Phase Behaviour of Surfactants, Academic Press: New York, 1994.

Leung, P. S. , Goddard, E. D. , Han, C. and Glinka, C. J. (1985), A study of polycation anionic surfactant systems, Colloids Surfaces 13, 47 - 62.

Li, Y. and Dubin, P. L. Havel, H.A., Edwards, S.L., Dautzenberg, H.(1995), Complex formation between polyelectrolyte and oppositely charged mixed micelles. Soluble complexes vs coacervation. Langmuir 11, 2486 - 2492.

Lindman, B. (1984), Structural aspects of surfactant micellar systems, in Surfactants, Tadros, Th. F. , Academic Press, New York, 1984, 83.

Lucassen, J. (1966), Hydrolysis and precipitation in carboxylate soap solutions, J. Phys. Chem. 70, $1824-1830$.

Madelmont, C. and Perron, R. (1976), Study of the influence of the chain length on some aspects of soap/water diagrams. Colloid Polym. Sci. 254, 581 - 595.

Magny, B. , Iliopoulos, I. , Zana, R. and Audebert, R. (1994), Mixed micelles formed by cationic surfactants and anionic hydrophobically modified polyelectrolytes, Langmuir 10, 3180 $-3187$.

Manning, G.S. (1977), Limiting laws and counterion condensation in polyelectrolyte solutions. IV. The approach to the limit and the extraordinary stability of the charge fraction, Biophys. Chem. 7, 95 - 102.

Manning, G.S. (1978), The molecular theory of polyelectrolyte solutions with applications to electrostatic properties of polynucleotides, Q. Rev. Biophys. 11, 179 - 246.

Manning, G.S. (1981), Limiting laws and counterion condensation in polyelectrolyte solutions. VII. Electrophoretic mobility and conductance. J. Phys. Chem. 85, 1506 - 1515.

Merta, J. and Stenius, P. (1995), Interactions between cationic starch and anionic surfactants. 1. Phase equilibria and surface temsions. Colloid Polym. Sci. 273, 974 - 983.

Merta, J. and Stenius, P.(1997), Interactions between cationic starch and anionic surfactants 2. Viscosity and aggregate size in dilute solutions. Colloids Surfaces A: Physicochem. Eng. Aspects 122, 243 - 255. 
Merta, J., Pirttinen, E., Stenius, P. (1999), Interactions between cationic starch and anionic surfactants. III. Rheology and structure of the complex phase. J. Dispersion Sci. and Techn. 20, 677 - 697.

Merta, J. and Stenius, P. (1999), Interactions between cationic starch and mixed anionic surfactants. Colloids Surfaces A: Physicochem. Eng. Aspects 149, 367 - 377.

Mitchell, D. J. and Ninham, B. W. (1981), Micelles, vesicles and microemulsions, J. Chem. Soc. Faraday Trans. 2, 77, 601 - 629.

Mukerjee, P. (1965), Dimerization of anions of long chain fatty acids in aqueous solutions and hydrophobic properties of the acids, J. Phys. Chem 69, 2821 - 2827.

Mukerjee, P. and Mysels, K. J. (1971), Critical Micelle Concentration of Aqueous Surfactant Systems, NSRDS-NBS 36, National Bureau of Standards, Washington D. C. , 1971.

Murata, M. and Arai, H. (1973), The interaction between polymer and surfactant. The effect of temperature and added salt on interaction between poly(vinylpyrrolidone) and sodium dodecyl sulfate, J. Colloid Interface Sci. 44, 475 - 480.

Musabekov, K. B. , Omarova, K. I. and Izimov, A. I. (1983), Modification of quartz surface with aqueous solutions of polyelectrolytes and surface active substances, Acta Phys. Chem. 29, $89-100$.

Nagarajan, R. and Kalpakci, B. (1982), Viscosimetric investigation of complexes between polyethylene oxide and surfactant micelles, Polym. Prepr. Am. Chem. Soc. Div. Polym. Chem. 23, $41-42$.

Nicolai, T. and Mandel, M. (1989), Dynamic light scattering by aqueous solutions of low molecular mass DNA fragments in the presence of NaCl, Macromolecules 22, 2348 - 2356.

Ober, C.K. and Wegner, G. (1997) Polyelectrolyte-surfactant complexis in solid state: Facile building blocks for self-organizing materials. Adv. Mater. 9, 17 - 31.

Odijk, T. (1978), On the theory of the excluded-volume effect of a polyelectrolyte in a 1-1 electrolyte solution, J. Polym. Sci. Polym. Phys. Ed. 16, 627 - 639. 
Ostanevich, Y. M. (1988), Time-of-flight small-angle scattering spectrometers on pulsed neutron sources. Makromol. Chem. Macromol. Symp. 15, 91 - 103.

Pecora, R., ed. (1985), Dynamic Light Scattering : Applications of Photon Correlation Spectroscopy, Plenum Press, New York, 1985.

Pedersen, J. S., Posselt, D., Mortensen, K. J. (1990) Analytical treatment of the resolution function of small-angle scattering. J. Appl. Cryst. 23, 321 - 333.

Penfold J., Staples E., Thompson L., Tucker I.; Hinea J.; Thomas R. K.; Lu J.R. (1995), Solution and adsorption behavior of the mixed surfactant system sodium dodecyl sulfate/n_hexaethylene glycol monododecyl ether. Langmuir 11, 2496 - 2503.

Penfold, J., Staples, E., Tucker, I. (1996), Study of partitioning in mixed surfactant systems, by neutron scattering techniques. Adv. Colloid Interface Sci. 68, 31 - 55.

Piculell, L.and Lindman, B. (1992), Association and segregation in aqueous polymer/polymer, polymer/surfactant and surfactant/surfactant mixtures: similarities and differences, Adv. Coll. Interface Sci. 41149 - 178.

Pilsl, H., Hoffmann, H., Hoffmann, J., Kalus, J., Kencono, A. W., Lindner, P., Ulbricht, W. (1993), Shape investigation of mixed micelles by small angle neutron scattering. J. Phys. Chem. 97, 2745 - 2754.

Prowencer, S. W. (1982), Contin: A general purpose constrained regularization program for inverting noisy linear algebraic and integral equations, Comput. Phys. Commun. 27, 229 - 242.

Ranganathan, S. and Kwak, J. T. C. (1996), Effect of polymer charge density on the phase behavior of sodium poly(acrylate_co_acrylamide)_DTAB systems. Langmuir 12, 1381 - 1390.

Rappennecker, G. and Zugenmaier, P. (1981) Detailed refinement of the crystal structure of $\mathrm{V}_{\mathrm{h}}$-amylose. Carbohydr. Res. 89, 11 - 19.

Robb, I. D. (1981), Polymer/surfactant interactions, in Anionic Surfactants - Physical Chemistry of Surfactant Action, Surfactant Sci. Ser., vol. 11, Lucassen-Reynders, E. , ed. , Marcel Dekker, New York, 1981, chap. 3.

Ruppelt, D., Kötz, J., Jaeger, W., Friberg, S.E., MacKay, R. (1997), Influence of cationic polyelectrolytes on structure formation in lamellar liquid crystalline systems. Langmuir 13, 3316 - 3319. 
Saito, S. (1991), Polymer-surfactant interactions, in Nonionic Surfactants, Schick, M.J., ed., Marcel Dekker, New York, 1991, 189.

Satake, I., Takahashi, T. , Hayakawa, K , Maeda, T. and Aoyagi, M. (1990), Effect of charge density on the cooperative binding isotherm of surfactant ion to polyelectrolyte, Bull.Chem. Soc. Jpn. 63, 926 - 928.

Schmitz, K. S. (1990), An Introduction to Dynamic Light Scattering by Macromolecules, Academic Press, San Diego, 1990.

Schurr, J. M. and Schmitz, K. S. (1986), Dynamic light scattering studies of biopolymers: effects of charge, shape and flexibility, Annu. Rev. Phys. Chem. 37, 271 - 305.

Sedlak, M. and Amis, E. J. (1992), Concentration and molecular weight-regime diagram of salt-free polyelectrolyte solutions as studied by light scattering, J. Chem. Phys. 96, 826 - 834.

Shuhrmann, H.B.; Burkhardt, N.; Dietrich; G.; Jünemann, R.; Meerwinck, W.; Schmitt, M.; Small-Angle X-ray Scattering; Glatter, O.; Kratky, O., Eds.; Academic Press: New York, 1982.

Smits, R. G., Kuil, M. E. and Mandel, M. (1993), Molar mass and ionic strength dependence of the apparent diffusion coeffient of a flexible polyelectrolyte at dilute and semidilute concentrations: linear poly(ethylenimine), Macromolecules 26, 6808 - 6816.

Snape, C. E., Morrison, W. R. , Marato-Valer, M. M. , Karkalas, J. and Pethrick, R. A. (1998), Solid state c nmr investigation of lipid ligands in v_amylose inclusion complexes. Carbohydrate Polymers 36, 225 - 237.

Sokolov, E.L., Yeh, F., Khokhlov, A. and Chu. B. (1996), Nanoscale supramolecular ordering in gel-surfactant complexes: sodium alkyl sulfates in poly(diallymethylammoinium chloride, Langmuir 12, 6229 - 6234.

Sokolov, E.L., Yeh, F., Khokhlov, A., Grinberg, V.Y. and Chu. B. (1998), Nanostructure formation in polyelectrolyte-surfactant complexes. J. Phys. Chem. B 102, 7091 - 7098.

Stigter, D. (1974), Micelle formation in ionic surfactants. II. Specificity of the head groups, micelle structure, J. Phys. Chem. 78, 2480 - 2485. 
Tadros, Th. F. (1974), The interaction of cetyltrimethylammonium bromide and sodium dodecylbenzen e sulfonate with polyvinyl alcohol. Adsorption of the polymer-surfactant complexes on silica, J. Colloid Interface Sci. 46, 528 - 540.

Taupin, D. and Luzzati, V. (1982) Information content and retrieval in solution scattering studies: 1. Degrees of freedom and data reduction, J. Appl. Cryst. 15, 289 - 300.

Thalberg, K. and Lindman, B. (1989), Interaction between hyalouran and cationic surfactants, J. Phys. Chem. 93, 1478 - 1483.

Thalberg, K., Lindman, B. and Karlström, G. (1990a), Phase diagram of a system of cationic surfactant and anionic polyelectrolyte: tetradecyltrimethylammonium bromide - hyalouran water, J. Phys. Chem. 94, 4289 - 4295.

Thalberg, K. (1990b), Polyelectrolyte-surfactant interactions, University of Lund, Lund 1990.

Thalberg, K., van Stam, J. , Lindblad, C. , Almgren, M. and Lindman, B.(1991 a), Timeresolved fluorescence and self-diffusion studies in systems of cationic surfactant and an anionic polyelectrolyte, J. Phys. Chem. 95, 8975 - 8982.

Thalberg, K. and Lindman, B.(1991b), Gel formation in aqueous systems of a polyanion and an oppositely charged surfactant, Langmuir 7, 277 - 283.

Thalberg, K., Lindman, B. and Bergfeld, K. (1991c), Phase behavior of systems of polyacrylate and cationic surfactant, Langmuir 7, 2893 - 2898.

Thalberg, K., Lindman, B. and Karlström (1991d), G., Phase diagram of systems of cationic surfactant and anionic polyelectrolyte: influence of surfactant chain length and polyelectrolyte molecular weight, J. Phys. Chem. 95, 3370 - 3376.

Thalberg, K., Lindman, B. and Karlström, G. (1991e), Phase diagram of systems of cationic surfactant and anionic polyelectrolyte: the effect of salt, J. Phys. Chem. 95, $6004-6011$.

Tomasik, P., Wang, V. and Jane, J. (1995), Complexes of starch with dioic acids. Starch 47, 91- 95.

van de Steeg, H.G.M. (1992), Cationic Starches on Cellulose Surfaces, University of Wageningen, Netherlands 1992. 
Verwey, E. J. W.; Overbeek, J. Th. G., Theory of the Stability of Lyophobic Colloids; Elsevier: Amsterdam, 1948.

Vold, R. D. (1939) The phase rule behavior of concentrated aqueous systems of a typical colloidal electrolyte: sodium oleate. J. Phys. Chem. 43, 1213 - 1231.

Wallin, T. and Linse, P. (1996), Monte Carlo simulations of polyelectrolytes at charged micelles. 2. Effects of linear charge density. J. Phys. Chem. 100, 17873 - 17880.

Wallin, T. and Linse, P. (1997), Monte Carlo simulations of polyelectrolytes at charged micelles. 3. Effects of surfactant tail length. J. Phys. Chem. B 101, 5506 - 5513.

Wang, L., Garner, M. M. and Yu, H.(1991), Self-diffusion and cooperative diffusion of a rodlike DNA fragments, Macromolecules 24, 2368 - 2376.

Wadzack, J., Willumeit, R., Zhao, J., Nierhaus, K.H. (1995) Proton- and deuteron spin targets in biological research, Nucl. Instr. Meth. A356, 124 -132.

Wenzel, A. and Antonietti, M. (1997), Superstructures of lipid bilayers by complexation with helical biopolymers. Adv. Mater. 9, 487 - 490.

Wignall, G.D. and Bates, F.S., (1986) Absolute calibration of small-angle neutron scattering data J. Appl. Cryst. 20, 28 - 40.

Yamamoto, M., Sano, T., Harada, S. and Yasunaga, T. (1983) Cooperativity in binding of sodium dedecyl sulfate to amylose. Bull. Chem. Soc. Jpn. 56, 2643 - 2646.

Zhang, K., Jonströmer, M. and Lindman, B. (1994a), Self-assembly in concentrated polymersurfactant systems - a self-diffusion study of a non-ionic random copolymer - non-ionic surfactant - water system, Colloids Surfaces 87, 133 - 142.

Zhang, K., Karlström, K. and Lindman, B. (1994b), Ternary aqueous mixtures of a nonionic polymer with a surfactant or a second polymer. A theoretical and experimental investigation of the phase behavior, J. Phys. Chem. 98, 4411 - 4421.

Zhang, K. Jonströmer, M. and Lindman, B. (1994c), Interaction between nonionic micelles and a nonionic polymer studied by Fourier transform NMR self-diffusion, J. Phys. Chem. 98, 2459 $-2463$. 
Zhang, K., Carlsson, M., Linse, P. and Lindman, B. (1994d), Phase behavior of copolymerhomopolymer mixture in aqueous solution, J. Phys. Chem. 98, 2452 - 2458.

Zhen, Z. and Tung, C.-H. (1992),, Interaction of sodium carboxymethylamylose with aqueous surfactants in both the presence and the absence of added salt: mixed micelles and inclusion complexes. Polymer 33, 812 - 816.

Zhou, S., Burger, C., Yeh, F., Chu, B (1998), Charge density effect of polyelectrolyte chains on the nanostructures of polyelectrolyte surfactant complexes, Macromolecules 31, 8157 8163.

Zhou, S., Yeh, F., Burger, C.; Chu, B.(1999), Formation and transition of highly ordered structures of polyelectrolyte-surfactant complexes. J. Phys. Chem. B 103, 2107 - 2112. 


\section{LABORATORY OF FOREST PRODUCTS CHEMISTRY \\ REPORTS, SERIES A}

1. Laine, J.,

Surface properties of unbleached kraft pulp fibres, determined by different methods. 142 p. 1994 .

2. Merta, J.,

Interactions between Cationic Starch and Anionic Surfactants. 148 p. 1995.

3. Heimonen, J.,

The effect of coating components and fillers in the flotation deinking of paper. 148 p. 1995.

4. Mitikka-Eklund, M.,

Sorption of xylans on cellulose fibres. 84 p. 1996.

5. Laurila, M.,

The adsorption of nonionic surfactants and polyacrylate on talc. 104 p. 1996.

6. Kekkonen, J.

Adhesional properties of polyamide 6 fibers used in press felts. 167 p. 1996

7. Laine, J.,

The effect of cooking and bleaching on the surface chemistry and charge properties of kraft pulp fibres. 199 p. 1996.

8. Vikkula, A.

Hemicelluloses in kraft cooking liquor. 81 p. 1999

9. Pirttinen, E.,

The effect of deinking chemicals in flotation deinking of paper. 50 p. 1999.

10. Vyörykkä, J.,

Konfokaali-raman-spektrometrin käyttö paperin päällysteen syvyyssuuntaiseen analysointiin. 83 p. 1999.

11. Saarinen, $T$.,

The surface properties of gels formed by cationic starch and surfactants. 109 p. 2000.

12. Merta, J.

Interactions between cationic starch and anionic surfactants. 107 p. 2001.

ISBN 951-22-5328-3

ISSN 1457-1382 